\title{
Thiophenols as Protic Nucleophilic Triggers in Aryne Three- Component Coupling
}

\author{
Subrata Bhattacharjee, Avishek Guin, Rahul N. Gaykar, and Akkattu T. Biju* \\ Department of Organic Chemistry, Indian Institute of Science, Bangalore- \\ 560012, India
}

Email:atbiju@iisc.ac.in

\section{Supporting Information}

1. General Information $\quad$ S02

2. General Procedure for the Optimization of the Reaction Conditions $\quad$ S03

3. General Procedure for the Thiophenol Triggered Aryne MCC with Aldehydes $\quad$ S04

4. Procedure for the Thiophenol Triggered Aryne MCC with $\alpha$-Keto Ester $\quad$ S05

5. Procedure for the Thiophenol Triggered Aryne MCC with

$\begin{array}{ll}\text { 2,2,2-Trifluoroacetophenone } & \text { S05 }\end{array}$

6. Mechanistic Experiments $\quad$ S06

7. X-Ray Data of 4ap $\quad$ S09

7. Synthesis and Characterization of 2-Arylthio Benzyl Alcohol Derivatives S10

8. Fe-Catalyzed Cyclisation of $\mathbf{4 a} \quad$ S36

9. ${ }^{1} \mathrm{H}$ and ${ }^{13} \mathrm{C}$ NMR Spectra of Products $\quad$ S37 


\section{General Information}

Unless otherwise specified, all reactions were carried out under an atmosphere of nitrogen in oven-dried reaction vessels with Teflon screw caps. The room temperature (rt) of the lab is $25^{\circ} \mathrm{C}$ when the experiments were carried out. THF was freshly purified by distillation over Na-benzophenone and was transferred under nitrogen. 18-Crown-6 was recrystallized from dry $\mathrm{CH}_{3} \mathrm{CN}$ and $\mathrm{KF}$ was dried by heating at $110{ }^{\circ} \mathrm{C}$ for $12 \mathrm{~h}$ and left to cool under nitrogen and stored in nitrogen filled glove-box. All the thiophenols, 2,2,2-trifluoro-1-phenylethan-1one 7a and ethyl 2-oxo-2-phenylacetate 5a were purchased from Alfa Aesar, TCI or SigmaAldrich and used as received. Deuterated 4-chlorobenzaldehyde ${ }^{1}$ and benzeneselenol ${ }^{2}$ were synthesized following the literature procedure. The 2(trimethylsilyl)phenyl trifluoromethane sulfonate 2a and the other symmetric and unsymmetrical aryne precursors were synthesized following literature procedure. ${ }^{3}$

Analytical thin layer chromatography was performed on TLC Silica gel $60 \mathrm{~F}_{254}$. Visualization was accomplished with short wave UV light or $\mathrm{KMnO}_{4}$ staining solutions followed by heating. Flash chromatography was performed on silica gel (230-400 mesh) by standard techniques eluting with Pet. Ether-EtOAc solvent system.

All compounds were fully characterized. ${ }^{1} \mathrm{H}$ and ${ }^{13} \mathrm{C}$ NMR spectra were recorded on Bruker Ultrashield spectrometer in $\mathrm{CDCl}_{3}$ as solvent. Chemical shifts $(\delta)$ are given in ppm. The residual solvent signals were used as references and the chemical shifts converted to the TMS scale $\left(\mathrm{CDCl}_{3}: \delta_{\mathrm{H}}=7.26 \mathrm{ppm}, \delta_{\mathrm{C}}=77.16 \mathrm{ppm}\right)$. Infrared (FT-IR) spectra were recorded on a Perkin Elmer Spectrum BX spectrophotometer, v-max in $\mathrm{cm}^{-1}$. HRMS (ESI) data were recorded on a Waters Xevo G2-XS Q-TOF instrument.

\footnotetext{
${ }^{1}$ Stahl, I. Chem. Ber. 1985, 118, 3166.

${ }^{2}$ Angeli, A.; Tanini, D.; Nocentini, A.; Capperucci, A.; Ferraroni, M.; Gratteri, P.; Supuran, C.T. Chem. Commun. 2019, 55, 648 .

${ }^{3}$ (a) Sato, Y.; Tamura, T.; Kinbara, A.; Morib, M Adv. Synth. Catal. 2007, 349, 647. (b) Peña, D.; Cobas, A.; Pérez, D.; Guitián, E. Synthesis, 2002, 1454.
} 


\section{General Procedure for the Optimization of the Reaction Conditions}<smiles>Cc1ccc(Br)c(N(C)c2ccc(Br)cc2)c1</smiles>

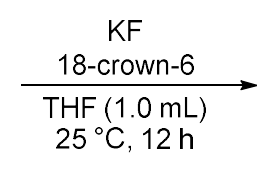<smiles>OC(c1ccc(Cl)cc1)c1ccccc1Sc1ccc(Br)cc1</smiles>

To an oven-dried screw-capped test tube equipped with a magnetic stir bar was added KF and 18-crown-6 in a glove-box. Then the 4-bromobenzenethiol $\mathbf{3 a}$ and 4chlorobenzaldehyde 1a $(0.035 \mathrm{~g}, 0.25 \mathrm{mmol})$ were added outside the glove-box under nitrogen atmosphere followed by addition of THF $(1 \mathrm{~mL})$. The reaction mixture was stirred for 10 minutes and then to the stirring solution, aryne precursor $\mathbf{2 a}$ was added and reaction mixture was allowed to stir at $25{ }^{\circ} \mathrm{C}$ for $12 \mathrm{~h}$. Yields were determined by the ${ }^{1} \mathrm{H}$ NMR analysis of the crude reaction products using $\mathrm{CH}_{2} \mathrm{Br}_{2}$ as the internal standard.

\begin{tabular}{|c|c|c|c|c|c|c|c|}
\hline entry & $\begin{array}{c}\mathbf{2 a} \\
\text { (equi } \\
\text { v) }\end{array}$ & $\begin{array}{c}\mathbf{3 a} \\
\text { (equiv) }\end{array}$ & $\mathrm{F}^{-}$source (equiv) & $\begin{array}{l}\text { Additive } \\
\text { (equiv) }\end{array}$ & Solvent & $\begin{array}{l}\text { Temp } \\
\left({ }^{\circ} \mathrm{C}\right)\end{array}$ & $\begin{array}{l}\text { Yield } \\
\text { of } 4 a \\
(\%)^{a}\end{array}$ \\
\hline 1 & 2 & 1.5 & KF (4), 18-crown-6 (4) & - & THF & $25^{\circ} \mathrm{C}$ & $42 \%$ \\
\hline 2 & 1.5 & 1.5 & KF (3), 18-crown-6 (3) & - & THF & $25^{\circ} \mathrm{C}$ & $39 \%$ \\
\hline 3 & 2.5 & 1.5 & KF (5), 18-crown-6 (5) & - & THF & $25^{\circ} \mathrm{C}$ & $40 \%$ \\
\hline 4 & 2 & 1.5 & $\mathrm{CsF}(4)$ & - & $\mathrm{CH}_{3} \mathrm{CN}$ & $25^{\circ} \mathrm{C}$ & $37 \%$ \\
\hline 5 & 2 & 1.5 & TBAF (4) & - & THF & $25^{\circ} \mathrm{C}$ & $6 \%$ \\
\hline 6 & 2 & 1.5 & KF (4), 18-crown-6 (4) & - & DME & $25^{\circ} \mathrm{C}$ & $22 \%$ \\
\hline 7 & 2 & 1.5 & KF (4), 18-crown-6 (4) & - & THF & $0^{\circ} \mathrm{C}$ & $41 \%$ \\
\hline 8 & 2 & 1.5 & KF (4), 18-crown-6 (4) & - & THF & $50^{\circ} \mathrm{C}$ & $34 \%$ \\
\hline 9 & 2 & 1.2 & KF (4), 18-crown-6 (4) & - & THF & $25^{\circ} \mathrm{C}$ & $30 \%$ \\
\hline 10 & 2 & 2 & KF (4), 18-crown-6 (4) & - & THF & $25^{\circ} \mathrm{C}$ & $77 \%$ \\
\hline 11 & 1.2 & 2 & KF (2.4), 18-crown-6 (2.4) & - & THF & $25^{\circ} \mathrm{C}$ & $21 \%$ \\
\hline 12 & 1.5 & 2 & KF (3), 18-crown-6 (3) & - & THF & $25^{\circ} \mathrm{C}$ & $31 \%$ \\
\hline 13 & 1.5 & 2 & KF (3), 18-crown-6 (3) & $\mathrm{K}_{2} \mathrm{CO}_{3}(2)$ & THF & $25^{\circ} \mathrm{C}$ & $53 \%$ \\
\hline 14 & 1.5 & 2 & KF (3), 18-crown-6 (3) & $\mathrm{K}_{2} \mathrm{CO}_{3}(3)$ & THF & $25^{\circ} \mathrm{C}$ & $48 \%$ \\
\hline 15 & 2 & 2 & KF (4), 18-crown-6 (4) & $\mathrm{K}_{2} \mathrm{CO}_{3}(2)$ & THF & $25^{\circ} \mathrm{C}$ & $69 \%$ \\
\hline 16 & 2 & 2 & KF (4), 18-crown-6 (4) & $\mathrm{Cs}_{2} \mathrm{CO}_{3}(2)$ & THF & $25^{\circ} \mathrm{C}$ & $53 \%$ \\
\hline 17 & 2 & 2 & KF (4), 18-crown-6 (6) & $\mathrm{K}_{2} \mathrm{CO}_{3}(2)$ & THF & $25^{\circ} \mathrm{C}$ & $67 \%$ \\
\hline 18 & 2 & 2 & KF (6), 18-crown-6 (6) & - & THF & $25^{\circ} \mathrm{C}$ & $94 \%$ \\
\hline 19 & 2 & 2 & KF (5), 18-crown-6 (5) & - & THF & $25^{\circ} \mathrm{C}$ & $94 \%$ \\
\hline 20 & 1.5 & 2 & KF (4), 18-crown-6 (4) & - & THF & $25^{\circ} \mathrm{C}$ & $58 \%$ \\
\hline 21 & 1.5 & 2 & KF (5), 18-crown-6 (5) & - & THF & $25^{\circ} \mathrm{C}$ & $64 \%$ \\
\hline 22 & 2 & 1.5 & KF (5), 18-crown-6 (5) & - & THF & $25^{\circ} \mathrm{C}$ & $47 \%$ \\
\hline
\end{tabular}

Standard conditions: 1a $(0.25 \mathrm{mmol})$, solvent $(1.0 \mathrm{~mL}), 12 \mathrm{~h} .{ }^{\text {a }}$ Yields based on the ${ }^{1} \mathrm{H}$ NMR analysis of the crude reaction products using $\mathrm{CH}_{2} \mathrm{Br}_{2}$ as the internal standard. 


\section{General Procedure for the Thiophenol Triggered Aryne MCC with}

\section{Aldehydes}

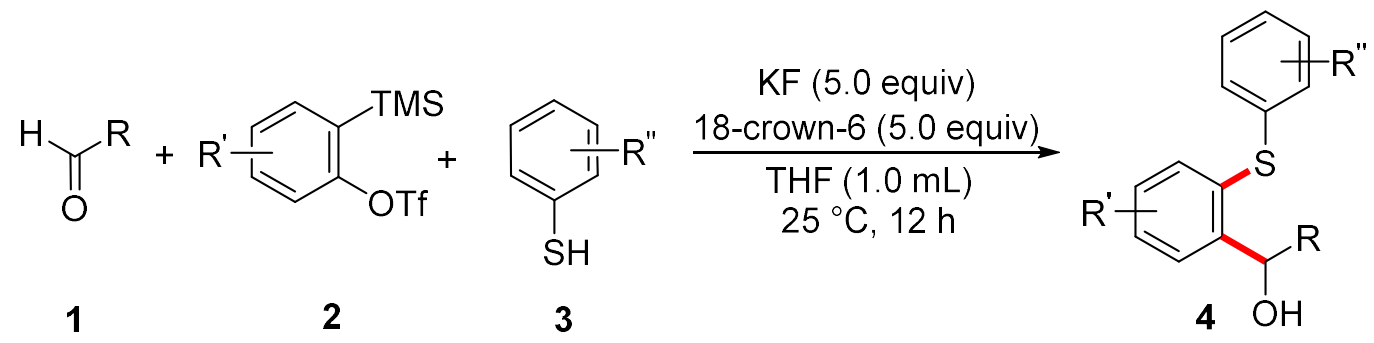

To an oven-dried screw-capped test tube equipped with a magnetic stir bar was added $\mathrm{KF}(0.073 \mathrm{~g}, 1.25 \mathrm{mmol})$ and 18 -crown-6 $(0.330 \mathrm{~g}, 1.25 \mathrm{mmol})$ in a glove-box. Then the thiophenol $3(0.5 \mathrm{mmol})$ and aldehyde $1(0.25 \mathrm{mmol})$ were added outside the glove-box under nitrogen atmosphere followed by addition of THF $(1 \mathrm{~mL})$. The reaction mixture was stirred for 10 minutes and then to the stirring solution, aryne precursor 2 ( 2.0 equiv, $0.5 \mathrm{mmol})$ was added and reaction mixture was allowed to stir at $25{ }^{\circ} \mathrm{C}$ for $12 \mathrm{~h}$. After completion of the reaction, solvent was evaporated and the crude residue was pre-adsorbed on silica gel and purified by flash column chromatography (Pet. ether $/$ EtOAc $=90 / 10$ ) on silica gel to afford the corresponding 2-arylthio benzyl alcohol derivatives $\mathbf{4}$ in moderate to good yields.

\section{Procedure for the 3.0 mmol Scale Reaction for the synthesis of 4 a}<smiles>O=Cc1ccc(Cl)cc1</smiles>

1a

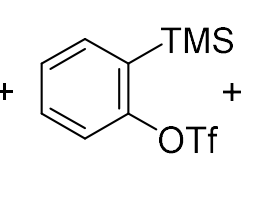

$2 a$<smiles>Sc1ccc(Br)cc1</smiles>

3a<smiles>OC(c1ccc(Cl)cc1)c1ccccc1Sc1ccc(Br)cc1</smiles>

To an oven-dried screw-capped test tube equipped with a magnetic stir bar was added KF (0.87 g, $15.0 \mathrm{mmol})$ and 18-crown-6 (3.96 g, $15.0 \mathrm{mmol})$ in a glove-box. Then 4bromobenzenethiol 3a $(1.134 \mathrm{~g}, 6.0 \mathrm{mmol})$ and 4-chlorobenzaldehyde 1a $(0.422 \mathrm{~g}, 3.0 \mathrm{mmol})$ were added outside the glove-box under nitrogen atmosphere followed by addition of THF (12 $\mathrm{mL}$ ). The reaction mixture was stirred for 15 minutes, and then to the stirring solution 2(trimethylsilyl)phenyl trifluoromethanesulfonate $\mathbf{2 a}(1.79 \mathrm{~g}, 6.0 \mathrm{mmol})$ was added and reaction mixture was allowed to stir at $25^{\circ} \mathrm{C}$ for $12 \mathrm{~h}$. After completion of the reaction, the solvent was evaporated and the crude residue was pre-adsorbed on silica gel and purified by flash column chromatography (Pet. ether /EtOAc $=90 / 10)$ on silica gel to afford (2-((4- 
bromophenyl)thio)phenyl)(4-chlorophenyl)methanol 4a as a colorless solid (1.114 g, 91\% yield).

\section{Procedure for the Thiophenol Triggered Aryne MCC with $\alpha$-Keto Ester}<smiles>CCOC(=O)C(=O)[PH2+]c1ccccc1[N+](=O)[O-]</smiles>

$5 a$<smiles>Sc1ccc(Br)cc1</smiles>

3a<smiles>CCOC(O)(c1ccccc1)c1ccccc1Sc1ccc(Br)cc1</smiles>

To an oven-dried screw-capped test tube equipped with a magnetic stir bar was added KF $(0.073 \mathrm{~g}, 1.25 \mathrm{mmol})$ and 18 -crown-6 $(0.330 \mathrm{~g}, 1.25 \mathrm{mmol})$ in a glove-box. Then the thiophenol 4-bromobenzenethiol 3a $(0.94 \mathrm{~g}, 0.5 \mathrm{mmol})$ and ethyl 2-oxo-2-phenylacetate 5a $(0.044 \mathrm{~g}, 0.25 \mathrm{mmol}, 40 \mu \mathrm{L})$ were added outside the glove-box under nitrogen atmosphere followed by addition of THF $(1 \mathrm{~mL})$. The reaction mixture was stirred for 10 minutes and then to the stirring solution 2-(trimethylsilyl)phenyl trifluoromethanesulfonate $\mathbf{2 a}(0.149 \mathrm{~g}, 121 \mu \mathrm{L}$, $0.5 \mathrm{mmol}$ ) was added, and reaction mixture was allowed to stir at $25{ }^{\circ} \mathrm{C}$ for $12 \mathrm{~h}$. After completion of the reaction, the solvent was evaporated and the crude residue pre-adsorbed on silica gel and purified by flash column chromatography (Pet. ether $/$ EtOAc $=90 / 10)$ on silica gel to afford ethyl 2-(2-((4-bromophenyl)thio)phenyl)-2-hydroxy-2-phenylacetate $\mathbf{6 a}$ as a colorless oil ( $0.078 \mathrm{~g}, 70 \%$ yield $)$.

\section{Procedure for the Thiophenol Triggered Aryne MCC with 2,2,2-}

\section{Trifluoroacetophenone}

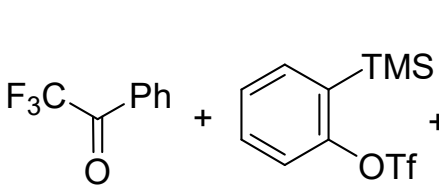

$7 a$

$2 a$<smiles>Sc1ccc(Br)cc1</smiles>

3a

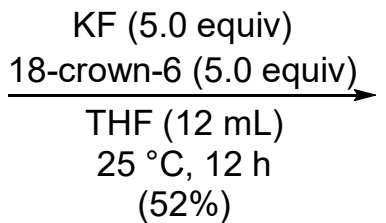

$(52 \%)$

To an oven-dried screw-capped test tube equipped with a magnetic stir bar was added KF $(0.073 \mathrm{~g}, 1.25 \mathrm{mmol})$ and 18 -crown-6 $(0.330 \mathrm{~g}, 1.25 \mathrm{mmol})$ in a glove-box. Then the thiophenol 4-bromobenzenethiol 3a (0.94 g, $0.5 \mathrm{mmol})$ and 2,2,2-trifluoro-1-phenylethan-1- 
one $7 \mathbf{a}(0.043 \mathrm{~g}, 0.25 \mathrm{mmol}, 35 \mu \mathrm{L})$ were added outside the glove-box under nitrogen atmosphere followed by addition of THF $(1 \mathrm{~mL})$. The reaction mixture was stirred for 10 minutes and then to the stirring solution 2-(trimethylsilyl)phenyl trifluoromethanesulfonate $\mathbf{2 a}$ $(0.149 \mathrm{~g}, 121 \mu \mathrm{L}, 0.5 \mathrm{mmol})$ was added, and reaction mixture was allowed to stir at $25^{\circ} \mathrm{C}$ for $12 \mathrm{~h}$. After completion of the reaction, the solvent was evaporated and the crude residue preadsorbed on silica gel and purified by flash column chromatography (Pet. ether /EtOAc $=$ 95/05) on silica gel to afford 1-(2-((4-bromophenyl)thio)phenyl)-2,2,2-trifluoro-1phenylethan-1-ol $8 \mathbf{a}$ as a colorless oil ( $0.057 \mathrm{~g}, 52 \%$ yield $)$.

\section{Mechanistic Experiments}

\section{Deuterium Labeling Experiment}<smiles>[2H]C(=O)c1ccc(Cl)cc1</smiles>

$>99 \% \mathrm{D}$

$D-1 \mathrm{a}$<smiles>CS(=O)(=O)c1ccccc1[OH+]</smiles>

2a<smiles>Sc1ccc(Br)cc1</smiles>

$3 a$

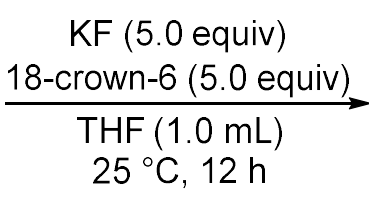

$25^{\circ} \mathrm{C}, 12 \mathrm{~h}$<smiles>O=C(O)C(O)(c1ccc(Cl)cc1)c1ccccc1Sc1ccc(Br)cc1</smiles>

$D-4 a$

Following the general procedure, treatment of deuterated 4-chlorobenzaldehyde $\boldsymbol{D}$-1a (0.035 g, $0.25 \mathrm{mmol}), 2$-(trimethylsilyl)phenyl trifluoromethanesulfonate $\mathbf{2 a}(0.149 \mathrm{~g}, 121 \mu \mathrm{L}$, $0.5 \mathrm{mmol})$ and 4-bromobenzenethiol 3a $(0.94 \mathrm{~g}, 0.5 \mathrm{mmol})$ in the presence of $\mathrm{KF}(0.073 \mathrm{~g}$, $1.25 \mathrm{mmol})$ and 18 -crown-6 $(0.330 \mathrm{~g}, 1.25 \mathrm{mmol})$ in THF $(1.0 \mathrm{~mL})$ at $25^{\circ} \mathrm{C}$ for $12 \mathrm{~h}$ followed by purification via silica gel flash column chromatography (Pet. ether $/$ EtOAc $=90 / 10)$ of the crude reaction mixture afforded (2-((4-bromophenyl)thio)phenyl)(4-chlorophenyl)methan-dol D-4a ( > 99 D) as a white solid (0.096 g, 94\% yield).

\section{${ }^{1} \mathrm{H}$ NMR spectrum of $D-4$ a}

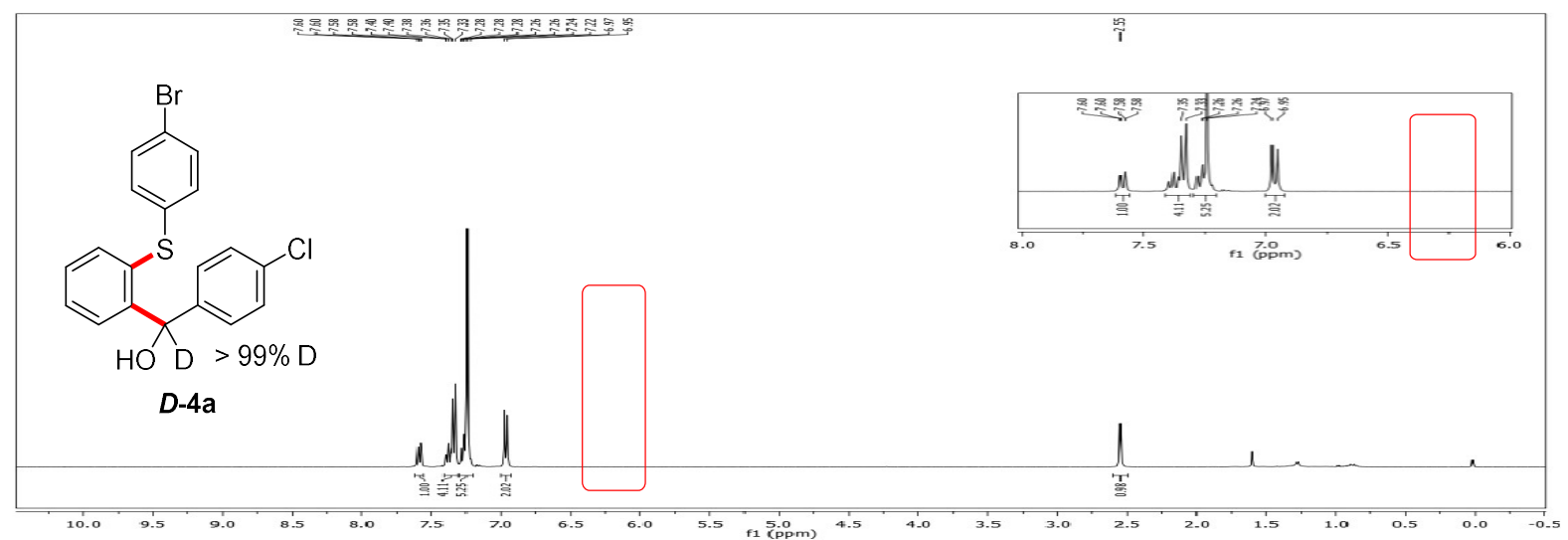


${ }^{1} \mathrm{H}$ NMR spectrum of $4 \mathrm{a}$

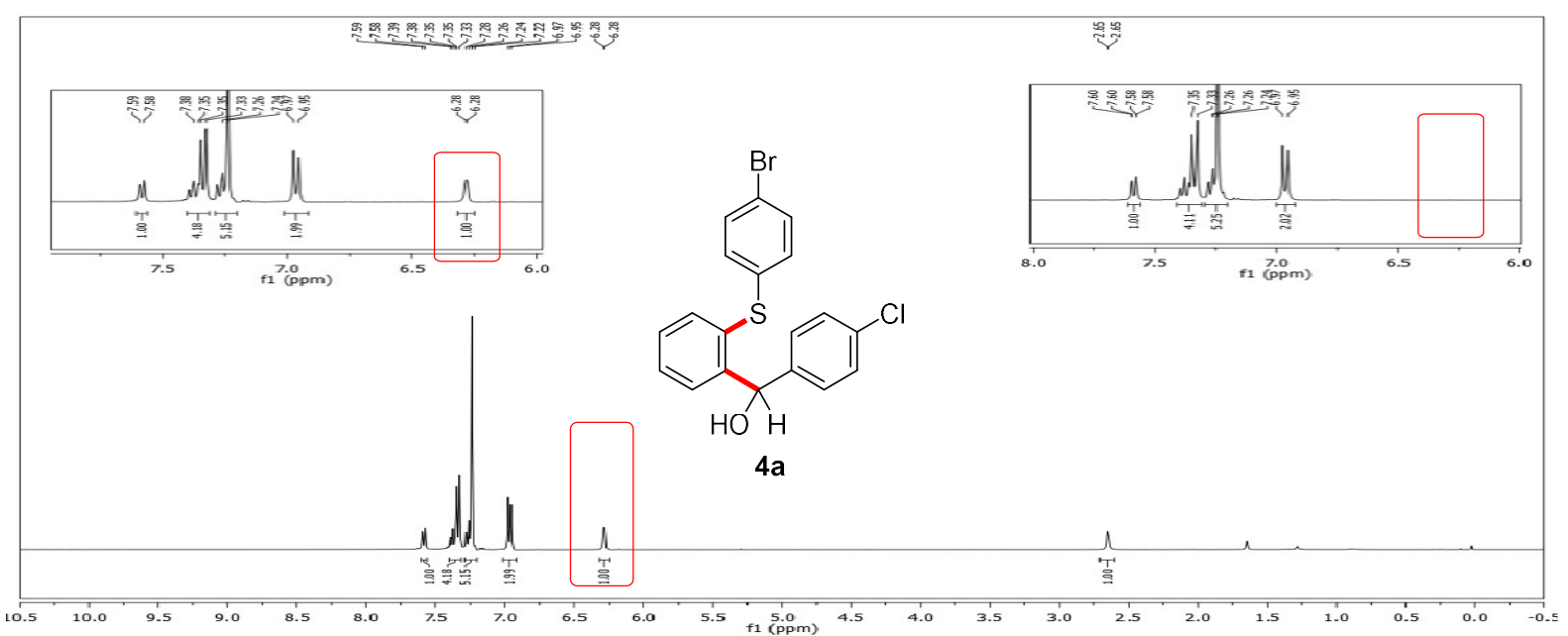

\section{${ }^{1} \mathrm{H}$ NMR spectrum of $D-1$ a}

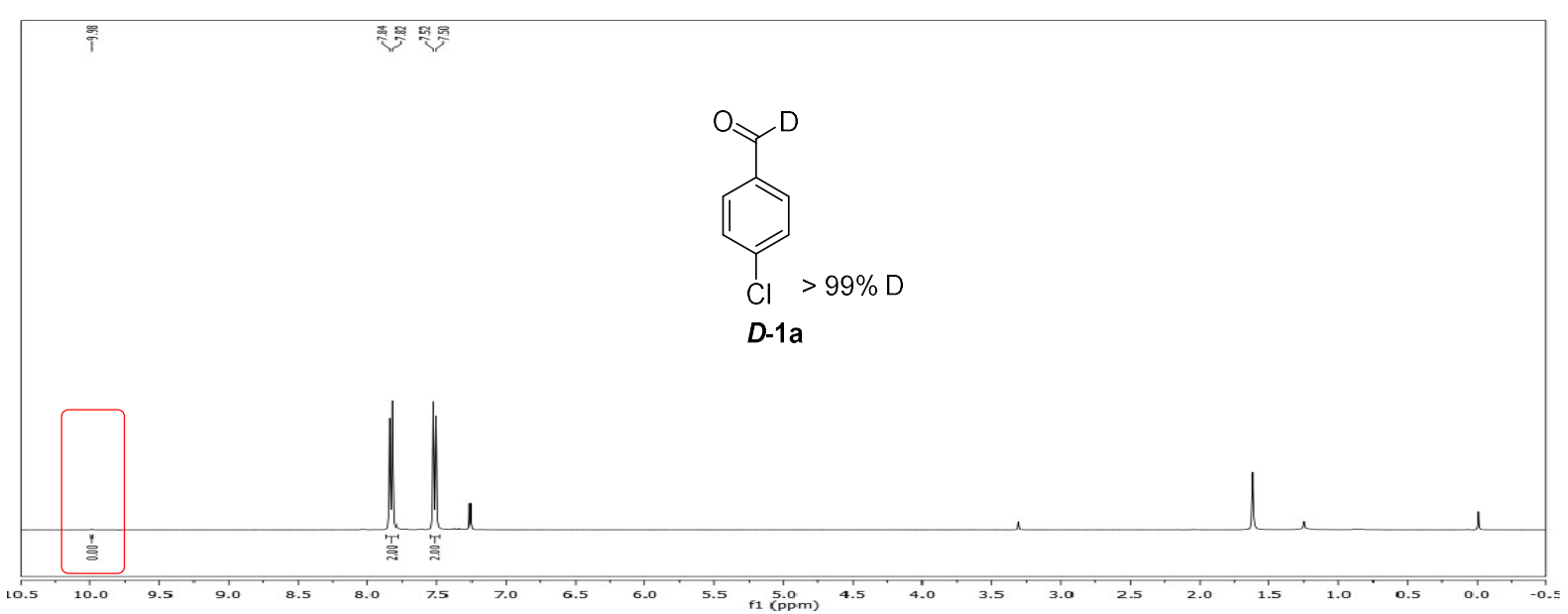

\section{Plausible Reaction Mechanism}

Mechanistically, the reaction proceeds via the generation of aryne from the triflate 2a in the presence of the KF/18-crown- 6 . The nucleophilic addition of the potassium thiolate (generated from the thiophenol using KF/18-crown-6) to the aryne results in the formation of the aryl anion intermediate 12, which adds to the aldehyde to form the alkoxide intermediate 13. This alkoxide $\mathbf{1 3}$ was subsequently protonated to afford the observed product $\mathbf{4 a}$. It is reasonable to assume that the $\mathrm{K}$-thiolate formed under the reaction conditions possibly drives towards the MCC product in preference to the simple S-H insertion product.

Given the fact that direct addition of thiophenol to aldehydes forming the adduct is a known process, ${ }^{4}$ we also considered the direct addition of thiophenol to aldehyde generating the hemithioacetal anion 14. A remote possibility was the proton transfer generating

\footnotetext{
${ }^{4}$ Bury, A.; Joag, S. D.; Stirling, C. J. M. J. Chem. Soc., Chem. Commun. 1986, 124.
} 
intermediate 15 (umpolung of aldehydes). This anion 15 can be converted into the desired product $\mathbf{4 a}$ via the addition to aryne generating the aryl anion $\mathbf{1 6}$ followed by the cleavage of C-S bond (very unlikely) to form the intermediate $\mathbf{1 7}$, which undergoes protonation to afford 4a. However, our preliminary mechanistic studies using deuterated 4-chlorobenzaldehyde $\boldsymbol{D}$ 1a rules out this possibility as the product $\boldsymbol{D}-\mathbf{- 4 a}$ was formed in $>99 \mathrm{D}$ incorporation.

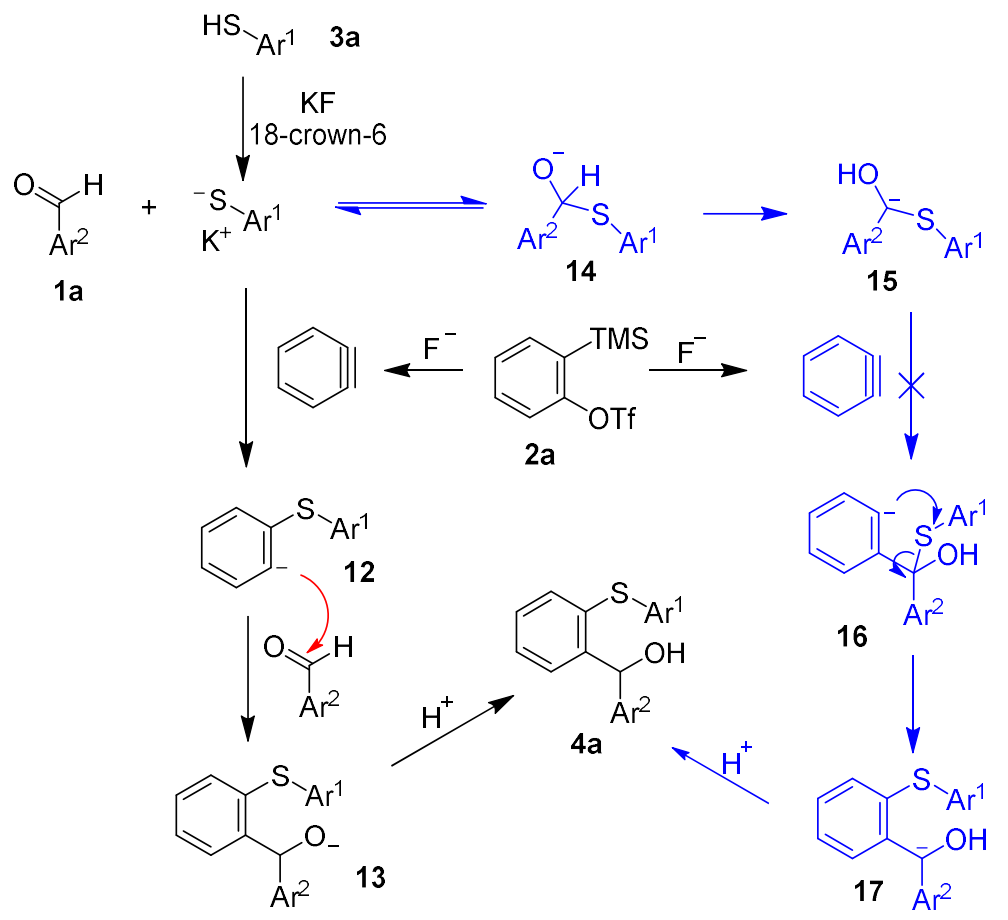

Additionally, when the 4-chlorobenzaldehyde 1a $(0.035 \mathrm{~g}, 0.25 \mathrm{mmol})$, and 4bromobenzenethiol 3a $(0.094 \mathrm{~g}, 0.5 \mathrm{mmol})$ were stirred in the presence of KF $(0.073 \mathrm{~g}, 1.25$ mmol) and 18-crown-6 $(0.330 \mathrm{~g}, 1.25 \mathrm{mmol})$ in THF $(1.0 \mathrm{~mL})$ for 10 minutes and checked TLC, new spots were observed along with the unreacted aldehyde and thiophenol. In GC, it was observed that $79 \%$ of the 4 -chlorobenzaldehyde $1 \mathrm{a}$ and $44 \%$ of the 4 -bromobenzenethiol 3a was remaining in the reaction mixture. This is may be due to the formation of potassium thiolate and aldehyde-thiophenol adduct. Then, we added the 2-(trimethylsilyl)phenyl trifluoromethanesulfonate $2 \mathrm{a}(0.149 \mathrm{~g}, 121 \mu \mathrm{L}, 0.5 \mathrm{mmol})$ and stirred the reaction mixture for $12 \mathrm{~h}$, interestingly, in TLC there was only the spots for $S$-arylation product, faint spot of aldehyde and spot for (2-((4-bromophenyl)thio)phenyl)(4-chlorophenyl)methanol 4a. Crude NMR of the reaction mixture indicated that $92 \%$ of the product $4 \mathbf{a}$ was formed. 


\section{X-Ray Data of 4ap}

Single crystals of 4ap (recrystallized from $\mathrm{CDCl}_{3}$ at $25^{\circ} \mathrm{C}$ ) was mounted and the diffraction data was collected at $293 \mathrm{~K}$ on a Bruker SMART APEX CCD diffractometer using SMART/SAINT software. Intensity data were collected using graphite-monochromatized MoKa radiation $(71.073 \mathrm{pm})$. The structure was solved by direct methods using the SHELX-97 5 and refined by full-matrix least-squares on F2. Empirical absorption corrections were applied with SADABS. ${ }^{6}$ All Non-hydrogen atoms were refined anisotropically and hydrogen atoms were included in geometric positions. Structure was drawn using Olex-2 and ORTEP-3. The crystallographic refinement parameters are given below:

\section{CCDC}

Identification code

Empirical formula

Formula weight

Temperature

Wavelength

Crystal system

Space group

Unit cell dimensions

Volume

Z

Density (calculated)

Absorption coefficient

$\mathrm{F}(000)$

Theta range for data collection

Index ranges

Reflections collected

Independent reflections

Completeness to theta $=25.242^{\circ}$

Refinement method

Data / restraints / parameters

Goodness-of-fit on $\mathrm{F}^{2}$

Final R indices [I $>2 \operatorname{sigma}(\mathrm{I})]$
2040671

4ap

$\mathrm{C}_{23} \mathrm{H}_{16} \mathrm{BrClOS}$

455.79

293(2) K

$0.71073 \AA$

Triclinic

$\mathrm{P}-1$

$\mathrm{a}=7.7020(2) \AA$ $\mathrm{a}=112.9500(10)^{\circ}$.

$\mathrm{b}=16.5408(4) \AA$ $\mathrm{b}=100.3150(10)^{\circ}$. $c=17.3416(4) \AA$ $1940.36(8) \AA^{3}$

4

$1.558 \mathrm{Mg} / \mathrm{m}^{3}$

$2.374 \mathrm{~mm}^{-1}$

918

2.283 to $30.524^{\circ}$.

$-11 \leq \mathrm{h} \leq 11,-23 \leq \mathrm{k} \leq 23,-24 \leq \mathrm{l} \leq 24$

44511

$11773[\mathrm{R}(\mathrm{int})=0.0361]$

$99.9 \%$

Full-matrix least-squares on $\mathrm{F}^{2}$

11773 / 0 / 495

0.995

$\mathrm{R} 1=0.0414, \mathrm{wR} 2=0.0885$

${ }^{5}$ G. M. SHELXL-2013 Sheldrick, University of Göttingen: Göttingen, Germany, 2014.

${ }^{6}$ G. M. Sheldrick, SADABS, University of Göttingen, Göttingen, Germany, 1999. 
$\mathrm{R}$ indices (all data)

$\mathrm{R} 1=0.0961, \mathrm{wR} 2=0.1056$

Extinction coefficient

$\mathrm{n} / \mathrm{a}$

Largest diff. peak and hole

0.345 and -0.411 e. $\AA^{-3}$

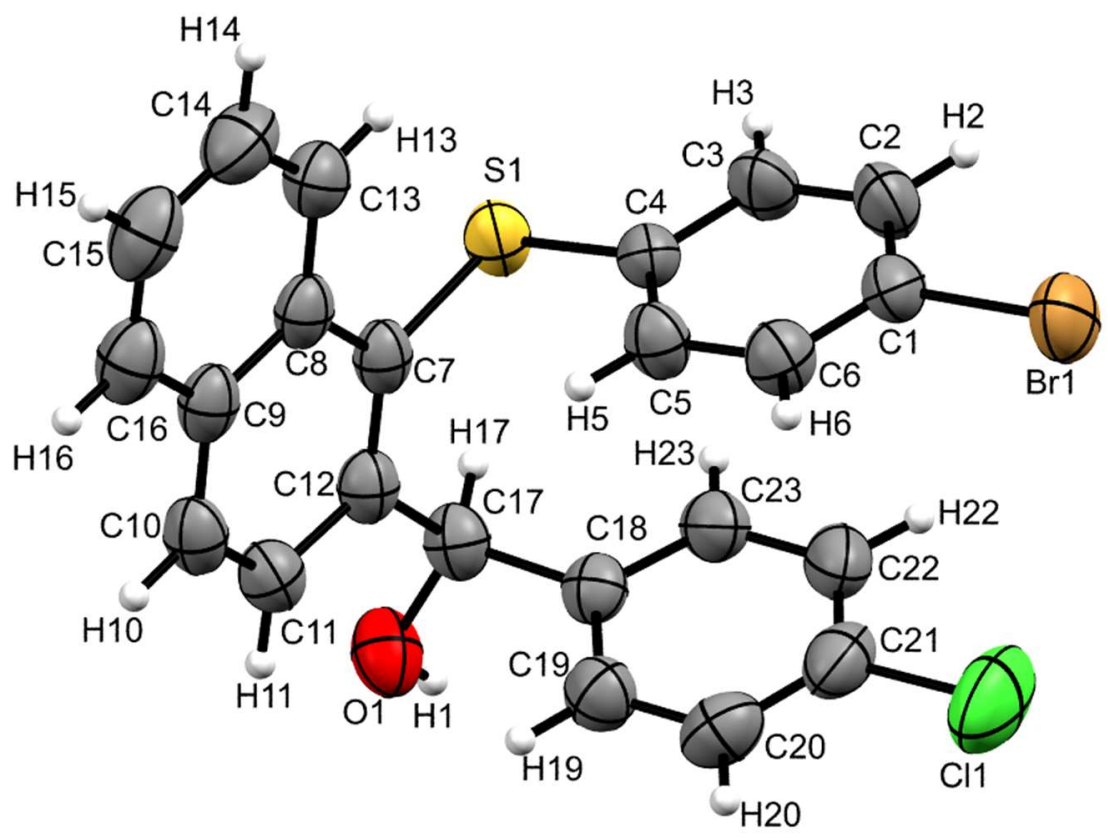

Ortep Diagram of 4ap (thermal ellipsoids are shown with 50\% probability).

\section{Synthesis and Characterization of 2-Arylthio Benzyl Alcohol Derivatives}

\section{(2-((4-Bromophenyl)thio)phenyl)(4-chlorophenyl)methanol (4a)}<smiles>OC(c1ccc(Cl)cc1)c1ccccc1Sc1ccc(Br)cc1</smiles>

$4 a$

Following the general procedure, treatment of 4-chlorobenzaldehyde 1a (0.07 g, $0.5 \mathrm{mmol})$, 2-(trimethylsilyl)phenyl trifluoromethanesulfonate 2a (0.298 g, $243 \mu \mathrm{L}, 1.0 \mathrm{mmol})$ and 4-bromobenzenethiol 3a (0.189 g, 1.0 $\mathrm{mmol})$ in the presence of KF $(0.145 \mathrm{~g}, 2.5 \mathrm{mmol})$ and 18-crown-6 (0.660 g, $2.5 \mathrm{mmol})$ in THF $(2.0 \mathrm{~mL})$ at $25^{\circ} \mathrm{C}$ for $12 \mathrm{~h}$ followed by purification via silica gel flash column chromatography $($ Pet. ether $/$ EtOAc $=90 / 05)$ of the crude reaction mixture afforded (2-((4-bromophenyl)thio)phenyl)(4-chlorophenyl)methanol 4a as a white solid $(0.187 \mathrm{~g}, 92 \%$ yield $)$.

$\boldsymbol{R}_{\mathbf{f}}($ Pet. ether $/$ EtOAc $=90 / 10)$ : 0.46; ${ }^{1} \mathbf{H}$ NMR (400 $\left.\mathbf{M H z}, \mathbf{C D C l}_{3}\right) \delta 7.58(\mathrm{~d}, J=7.8 \mathrm{~Hz}, 1 \mathrm{H})$, 7.39-7.33 (m, 4H), 7.28-7.22 (m, 5H), 6.96 (d, $J=8.5 \mathrm{~Hz}, 2 \mathrm{H}), 6.28(\mathrm{~d}, J=1.7 \mathrm{~Hz}, 1 \mathrm{H}), 2.65$ $(\mathrm{d}, J=2.6 \mathrm{~Hz}, 1 \mathrm{H}) .{ }^{13} \mathbf{C} \mathbf{N M R}\left(\mathbf{1 0 0} \mathbf{M H z}, \mathbf{C D C l}_{3}\right) \delta 145.1,141.3,135.7,134.6,133.5,132.3$, 131.8, 131.0, 129.1, 128.9, 128.6, 128.5, 127.9, 120.6, 72.8. HRMS (ESI) m/z: [M+Na] ${ }^{+}$calcd 
for (mass of corresponding oxidized product using PDC) $\mathrm{C}_{19} \mathrm{H}_{12} \mathrm{BrClNaOS} 424.9373$; found 424.9382. FTIR (cm-1) 3578, 3383, 3057, 2922, 1592, 1492, 1437, 1384, 1179, 1089, 1009.

\section{2-((4-Bromophenyl)thio)phenyl)(4-methoxyphenyl)methanol (4b) ${ }^{7}$}<smiles>COc1ccc(C(O)c2ccccc2Sc2ccc(Br)cc2)cc1</smiles>

Following the general procedure, treatment of 4methoxybenzaldehyde $\mathbf{1 b}(0.034 \mathrm{~g}, 0.25 \mathrm{mmol})$, 2-(trimethylsilyl) phenyl trifluoromethanesulfonate $2 \mathrm{a}(0.149 \mathrm{~g}, 121 \mu \mathrm{L}, 0.5 \mathrm{mmol})$ and 4-bromobenzenethiol 3a $(0.095 \mathrm{~g}, 0.5 \mathrm{mmol})$ in the presence of $\mathrm{KF}$ (0.073 g, $1.25 \mathrm{mmol})$ and 18-crown-6 (0.330 g, $1.25 \mathrm{mmol})$ in THF $(1.0 \mathrm{~mL})$ at $25^{\circ} \mathrm{C}$ for $12 \mathrm{~h}$ followed by purification via silica gel flash column chromatography (Pet. ether $/$ EtOAc $=88 / 12)$ of the crude reaction mixture afforded 2-((4-bromophenyl)thio)phenyl)(4-methoxyphenyl)methanol $\mathbf{4 b}$ as a white solid (0.075 g, 75\% yield).

$\boldsymbol{R}_{\mathbf{f}}($ Pet. ether $/$ EtOAc $=90 / 10): 0.32 ;{ }^{1} \mathbf{H}$ NMR (400 MHz, $\left.\mathbf{C D C l}_{3}\right) \delta 7.70(\mathrm{dd}, J 1=7.8 \mathrm{~Hz}, J 2$ $=1.3 \mathrm{~Hz}, 1 \mathrm{H}), 7.41-7.37(\mathrm{~m}, 1 \mathrm{H}), 7.33-7.28(\mathrm{~m}, 3 \mathrm{H}), 7.26-7.24(\mathrm{~m}, 1 \mathrm{H}), 7.22-7.20(\mathrm{~m}, 2 \mathrm{H})$, 6.95-6.92 (m, 2H), 6.80-6.77 (m, 2H), 6.26 (d, $J=3.4 \mathrm{~Hz}, 1 \mathrm{H}), 3.77$ (s, 3H), 2.48 (d, $J=4.1$ $\mathrm{Hz}, 1 \mathrm{H}) .{ }^{13} \mathbf{C}$ NMR (100 MHz, CDCl 3$) \delta 159.14,145.72,136.06,135.05,134.59,132.17$, 131.57, 130.90, 128.93, 128.54, 127.60, 120.31, 113.90, 73.12, 55.37. HRMS (ESI) m/z: $[\mathrm{M}+\mathrm{Na}]^{+}$calcd for (mass of corresponding oxidized product using PDC) $\mathrm{C}_{20} \mathrm{H}_{15} \mathrm{BrNaO}_{2} \mathrm{~S}$ 420.9868; found 420.9873. FTIR ( $\left.\mathbf{c m}^{-1}\right)$ 3744, 3649, 3395, 2928, 1611, 1511, 1467, 1435 , $1248,1006$.

\section{(2-((4-Bromophenyl)thio)phenyl)(p-tolyl)methanol (4c) ${ }^{7}$}

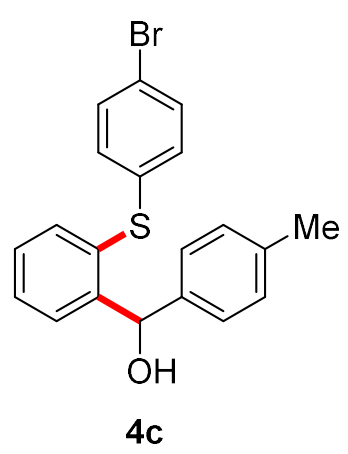

Following the general procedure, treatment of 4-methylbenzaldehyde 1c $(0.030 \mathrm{~g}, \quad 0.25 \mathrm{mmol}), \quad 2-($ trimethylsilyl)phenyl trifluoromethane sulfonate $2 \mathbf{a}(0.149 \mathrm{~g}, 121 \mu \mathrm{L}, 0.5 \mathrm{mmol})$ and 4-bromobenzenethiol 3a $(0.095 \mathrm{~g}, 0.5 \mathrm{mmol})$ in the presence of $\mathrm{KF}(0.073 \mathrm{~g}, 1.25 \mathrm{mmol})$ and 18 crown-6 (0.330 g, $1.25 \mathrm{mmol})$ in THF $(1.0 \mathrm{~mL})$ at $25^{\circ} \mathrm{C}$ for $12 \mathrm{~h}$ followed by purification via silica gel flash column chromatography (Pet. ether $/$ EtOAc $=94 / 6)$ of the crude reaction mixture afforded (2-((4bromophenyl)thio)phenyl)( $p$-tolyl)methanol $4 \mathbf{c}$ as a white solid (0.075 g, $78 \%$ yield).

\footnotetext{
${ }^{7}$ Yildiz, T. Synth. Commun. 2018, 48, 2177.
} 
$\boldsymbol{R}_{\mathbf{f}}($ Pet. ether $/$ EtOAc $=90 / 10)$ : 0.48; ${ }^{1} \mathbf{H}$ NMR (400 $\left.\mathbf{M H z}, \mathbf{C D C l}_{3}\right) \delta 7.71(\mathrm{~d}, J=7.8 \mathrm{~Hz}, 2 \mathrm{H})$, $7.42(\mathrm{t}, J=7.7 \mathrm{~Hz}, 1 \mathrm{H}), 7.37-7.33(\mathrm{~m}, 3 \mathrm{H}), 7.29-7.26(\mathrm{~m}, 1 \mathrm{H}), 7.23$ (d, $J=8.0 \mathrm{~Hz}, 2 \mathrm{H}), 7.11$ $(\mathrm{d}, J=7.8 \mathrm{~Hz}, 1 \mathrm{H}), 6.99(\mathrm{~d}, J=8.6 \mathrm{~Hz}, 2 \mathrm{H}), 6.31$ (d, $J=3.0 \mathrm{~Hz}, 1 \mathrm{H}), 2.54(\mathrm{~s}, 1 \mathrm{H}) 2.35(\mathrm{~s}, 3 \mathrm{H})$. ${ }^{13}$ C NMR (100 MHz, CDCl3) $\delta$ 145.70, 139.92, 137.44, 136.09, 134.59, 132.17, 131.66, 130.90, 129.20, 128.94, 128.55, 127.81, 127.11, 120.33, 73.33, 21.23. HRMS (ESI) m/z: $[\mathrm{M}+\mathrm{Na}]^{+}$calcd for (mass of corresponding oxidized product using PDC) $\mathrm{C}_{20} \mathrm{H}_{15} \mathrm{BrNaOS}$ 404.9919; found 404.9928. FTIR (cm $\left.{ }^{-1}\right)$ 3744, 3442, 3056, 1647, 1564, 1513, 1468, 1431, $1004,807$.

\section{(2-((4-Bromophenyl)thio)phenyl)(phenyl)methanol (4d)}

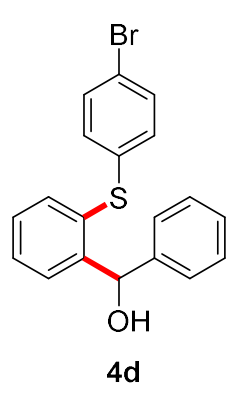

Following the general procedure, treatment of benzaldehyde $\mathbf{1 d}(0.026 \mathrm{~g}, 0.25$ mmol, $25 \mu \mathrm{L}), 2$-(trimethylsilyl)phenyl trifluoromethanesulfonate $\mathbf{2 a}(0.149 \mathrm{~g}$, $121 \mu \mathrm{L}, 0.5 \mathrm{mmol})$ and 4-bromobenzenethiol 3a $(0.094 \mathrm{~g}, 0.5 \mathrm{mmol})$ in the presence of KF (0.073 g, $1.25 \mathrm{mmol})$ and 18-crown-6 (0.330 g, $1.25 \mathrm{mmol})$ in THF $(1.0 \mathrm{~mL})$ at $25^{\circ} \mathrm{C}$ for $12 \mathrm{~h}$ followed by purification via silica gel flash column chromatography $($ Pet. ether $/$ EtOAc $=90 / 10)$ of the crude reaction mixture afforded (2-((4-bromophenyl)thio)phenyl)(phenyl)methanol $\mathbf{4 d}$ as a colorless oil (0.057 g, 62\% yield).

$\boldsymbol{R} \mathbf{f}($ Pet. ether $/$ EtOAc $=90 / 10): 0.45 ;{ }^{1} \mathbf{H}$ NMR (400 MHz, CDCl $) \delta 7.65\left(\mathrm{dd}, J_{1}=7.8 \mathrm{~Hz}, J_{2}\right.$ $=1.0 \mathrm{~Hz}, 1 \mathrm{H}), 7.40-7.23(\mathrm{~m}, 10 \mathrm{H}), 6.99-6.97(\mathrm{~m}, 2 \mathrm{H}), 6.34(\mathrm{~d}, J=1.5 \mathrm{~Hz}, 1 \mathrm{H}), 2.53(\mathrm{~d}, J=$ $3.1 \mathrm{~Hz}, 1 \mathrm{H}) .{ }^{13} \mathbf{C}$ NMR (100 MHz, CDCl$) \delta 145.5,142.9,136.0,134.5,132.3,131.9,131.0$, 129.0, 128.7, 128.6, 128.0, 127.7, 127.1, 120.5, 73.5. HRMS (ESI) m/z: $[\mathrm{M}+\mathrm{H}]^{+}$calcd for (mass of corresponding oxidized product using PDC) $\mathrm{C}_{19} \mathrm{H}_{14} \mathrm{BrOS} 368.9943$; found 368.9945 . FTIR (cm $\left.{ }^{-1}\right)$ 3384, 3058, 2365, 1567, 1468, 1385, 1180, 1089, 1006.

\section{4-((2-((4-Bromophenyl)thio)phenyl)(hydroxy)methyl)benzonitrile (4e)}

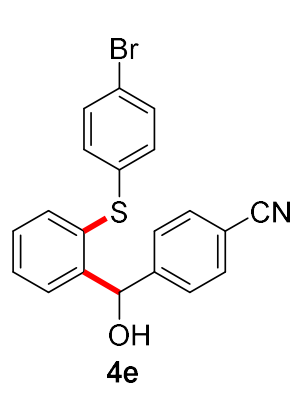

Following the general procedure, treatment of 4-formylbenzonitrile 1e (0.033 g, $0.25 \mathrm{mmol}), 2$-(trimethylsilyl)phenyl trifluoromethanesulfonate 2a $(0.149 \mathrm{~g}, 121 \mu \mathrm{L}, 0.5 \mathrm{mmol})$ and 4-bromobenzenethiol 3a (0.094 g, 0.5 $\mathrm{mmol})$ in the presence of $\mathrm{KF}(0.073 \mathrm{~g}, 1.25 \mathrm{mmol})$ and 18-crown-6 (0.330 $\mathrm{g}, 1.25 \mathrm{mmol})$ in THF $(1.0 \mathrm{~mL})$ at $25^{\circ} \mathrm{C}$ for $12 \mathrm{~h}$ followed by purification via silica gel flash column chromatography $($ Pet. ether $/$ EtOAc $=90 / 10)$ of 
the crude reaction mixture afforded 4-((2-((4-bromophenyl)thio)phenyl)(hydroxy)methyl) benzonitrile $4 \mathbf{e}$ as a colorless sticky liquid $(0.081 \mathrm{~g}, 82 \%$ yield).

$\boldsymbol{R}_{\mathbf{f}}($ Pet. ether $/ \mathrm{EtOAc}=90 / 10): 0.43 ;{ }^{1} \mathbf{H}$ NMR (400 MHz, $\left.\mathbf{C D C l}_{3}\right) \delta$ 7.56-7.50 (m, 3H), 7.45$7.43(\mathrm{~m}, 2 \mathrm{H}), 7.40-7.33(\mathrm{~m}, 4 \mathrm{H}), 7.30-7.26(\mathrm{~m}, 1 \mathrm{H}), 6.99-6.96(\mathrm{~m}, 2 \mathrm{H}), 6.37(\mathrm{~s}, 1 \mathrm{H}), 2.77$ (s, 1H). ${ }^{13} \mathrm{C}$ NMR (100 MHz, CDCl 3$) \delta 148.1,144.5,135.4,134.7,132.4,132.3,131.9,131.0$, 129.3, 129.3, 128.2, 127.6, 120.8, 118.8, 111.3, 72.7. HRMS (ESI) m/z: $[\mathrm{M}+\mathrm{Na}]^{+}$calcd for $\mathrm{C}_{20} \mathrm{H}_{14} \mathrm{BrNNaOS}$ 417.9872; found 417.9876. FTIR (cm $\left.{ }^{-1}\right)$ 3461, 2922, 1607, 1469, 1384, 1186, 1088, 1007.

\section{Methyl-4-((2-((4-bromophenyl)thio)phenyl)(hydroxy)methyl)benzoate (4f)}<smiles>CC(=O)c1ccc(C(O)c2ccccc2Sc2ccc(Br)cc2)cc1</smiles>

Following the general procedure, treatment of methyl 4formylbenzoate $\mathbf{1 f}(0.041 \mathrm{~g}, 0.25 \mathrm{mmol})$, 2-(trimethylsilyl)phenyl trifluoromethanesulfonate $2 \mathrm{a}(0.149 \mathrm{~g}, 121 \mu \mathrm{L}, 0.5 \mathrm{mmol})$ and 4bromobenzenethiol 3a $(0.095 \mathrm{~g}, 0.5 \mathrm{mmol})$ in the presence of $\mathrm{KF}$ (0.073 g, $1.25 \mathrm{mmol})$ and 18-crown-6 (0.330 g, $1.25 \mathrm{mmol})$ in THF $(1.0 \mathrm{~mL})$ at $25^{\circ} \mathrm{C}$ for $12 \mathrm{~h}$ followed by purification via silica gel flash column chromatography $($ Pet. ether $/$ EtOAc $=92 / 8)$ of the crude reaction mixture afforded methyl-4-((2-((4-bromophenyl)thio)phenyl)(hydroxy)methyl) benzoate $4 \mathbf{f}$ as a white solid ( $0.100 \mathrm{~g}, 93 \%$ yield).

$\boldsymbol{R}_{\mathbf{f}}\left(\right.$ Pet. ether /EtOAc = 90/10): 0.37; ${ }^{1} \mathbf{H}$ NMR (400 MHz, $\left.\mathbf{C D C l}_{3}\right) \delta 7.93(\mathrm{~d}, J=8.0 \mathrm{~Hz}, 2 \mathrm{H})$, $7.59(\mathrm{~d}, J=7.7 \mathrm{~Hz}, 1 \mathrm{H}), 7.41-7.26(\mathrm{~m}, 7 \mathrm{H}), 6.97(\mathrm{~d}, J=8.0 \mathrm{~Hz}, 1 \mathrm{H}), 6.39(\mathrm{~s}, 1 \mathrm{H}), 3.90(\mathrm{~s}, 3 \mathrm{H})$, 3.00 (s, 1H). ${ }^{13} \mathbf{C}$ NMR (100 MHz, CDCl 3$) \delta 167.0,147.9,145.1,135.8,134.7,132.3,131.8$, 130.8, 129.8, 129.2 129.2, 129.0, 128.2, 126.9, 120.5, 73.0, 52.2. HRMS (ESI) m/z: $[\mathrm{M}+\mathrm{Na}]^{+}$ calcd for $\mathrm{C}_{21} \mathrm{H}_{17} \mathrm{BrNaO}_{3} \mathrm{~S}$ 450.9979; found 450.9975. FTIR (cm $\left.{ }^{-1}\right)$ 3479, 3058, 1722, 1611, 1469, 1437, 1283, 1184, 1113, 1010.

\section{4-((2-((4-Bromophenyl)thio)phenyl)(hydroxy)methyl)benzaldehyde (4g)}

Following the general procedure, treatment of terephthalaldehyde $1 \mathrm{~g}(0.035 \mathrm{~g}, 0.25 \mathrm{mmol}), 2-$ (trimethylsilyl)phenyl trifluoromethane sulfonate 2a $(0.149 \mathrm{~g}, 121 \mu \mathrm{L}, 0.5 \mathrm{mmol})$ and 4bromobenzenethiol 3a $(0.095 \mathrm{~g}, 0.5 \mathrm{mmol})$ in the presence of KF $(0.073 \mathrm{~g}, 1.25 \mathrm{mmol})$ and 18 crown-6 $(0.330 \mathrm{~g}, 1.25 \mathrm{mmol})$ in THF $(1.0 \mathrm{~mL})$ at $25^{\circ} \mathrm{C}$ for $12 \mathrm{~h}$ followed by purification via silica gel flash column chromatography $($ Pet. ether $/$ EtOAc $=80 / 20)$ of the crude reaction 
mixture afforded 4-((2-((4-bromophenyl)thio)phenyl)(hydroxy)methyl)benzaldehyde $\mathbf{4 g}$ as a white solid (0.052 g, 52\% yield).

$\boldsymbol{R}_{\mathbf{f}}($ Pet. ether $/$ EtOAc $=90 / 10):$ 0.29; ${ }^{1} \mathbf{H}$ NMR (400 MHz, $\left.\mathbf{C D C l}_{3}\right) \delta 9.94(\mathrm{~s}, 1 \mathrm{H}), 7.77(\mathrm{~d}, J=$<smiles>O=Cc1ccc(C(O)c2ccccc2Sc2ccc(Br)cc2)cc1</smiles>
$8.3 \mathrm{~Hz}, 2 \mathrm{H}), 7.56(\mathrm{dd}, J 1=7.8 \mathrm{~Hz}, J 2=1.2 \mathrm{~Hz}, 1 \mathrm{H}), 7.49$ (d, $J=8.2 \mathrm{~Hz}$, 2H), 7.40-7.27 (m, 5H), $6.96(\mathrm{~d}, J=8.6 \mathrm{~Hz}, 1 \mathrm{H}), 6.40(\mathrm{~d}, J=2.8 \mathrm{~Hz}, 1 \mathrm{H})$, $2.80(\mathrm{~d}, J=3.8 \mathrm{~Hz}, 1 \mathrm{H}) .{ }^{\mathbf{1 3}} \mathbf{C} \mathbf{N M R}\left(\mathbf{1 0 0} \mathbf{M H z}, \mathbf{C D C l}_{3}\right) \delta 192.05,149.58$, $144.87,135.65,134.82,132.38,131.87,130.89,129.97,129.30,129.16$, 128.25, 127.54, 120.69, 73.02. HRMS (ESI) m/z: [M] $]^{+}$calcd for (mass of corresponding oxidized product using PDC) $\mathrm{C}_{20} \mathrm{H}_{13} \mathrm{BrO}_{2} \mathrm{~S} 395.9820$; found 395.9820. FTIR (cm-1) 3441, 3057, 2829, 1697, 1606, 1575, 1469, 1434, 1387, 1212.

\section{(2-((4-Bromophenyl)thio)phenyl)(m-tolyl)methanol (4h)}

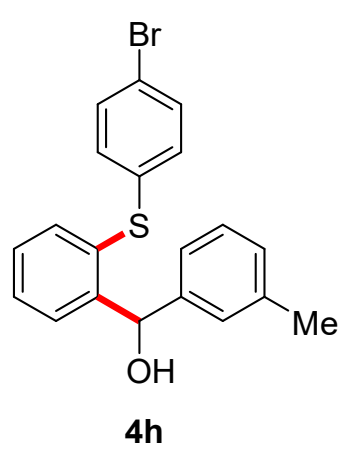

Following the general procedure, treatment of 3-methylbenzaldehyde $\mathbf{1 h}$ (0.030 g, $0.25 \mathrm{mmol}), \quad$ 2-(trimethylsilyl)phenyl trifluoromethane sulfonate 2a $(0.149 \mathrm{~g}, 121 \mu \mathrm{L}, 0.5 \mathrm{mmol})$ and 4-bromobenzenethiol 3a $(0.095 \mathrm{~g}, 0.5 \mathrm{mmol})$ in the presence of $\mathrm{KF}(0.073 \mathrm{~g}, 1.25 \mathrm{mmol})$ and 18 crown-6 (0.330 g, $1.25 \mathrm{mmol})$ in THF $(1.0 \mathrm{~mL})$ at $25^{\circ} \mathrm{C}$ for $12 \mathrm{~h}$ followed by purification via silica gel flash column chromatography (Pet. ether $/$ EtOAc $=93 / 7)$ of the crude reaction mixture afforded $(2-((4-$ bromophenyl)thio)phenyl)( $m$-tolyl)methanol $\mathbf{4 h}$ as a white solid ( $0.86 \mathrm{~g}, 89 \%$ yield).

$\boldsymbol{R}_{\mathbf{f}}($ Pet. ether $/$ EtOAc $=90 / 10): 0.43 ;{ }^{1} \mathbf{H}$ NMR (400 MHz, $\left.\mathbf{C D C l}_{3}\right) \delta 7.66(\mathrm{~d}, J=8.0 \mathrm{~Hz}, 1 \mathrm{H})$, 7.41-7.31 (m, 4H), 7.28-7.24 (m, 1H), 7.20-7.10 (m, 3H), 7.05 (d, $J=7.3 \mathrm{~Hz}, 2 \mathrm{H}), 6.96(\mathrm{~d}, J$ $=8.4 \mathrm{~Hz}, 1 \mathrm{H}), 6.31(\mathrm{~d}, J=3.4 \mathrm{~Hz}, 1 \mathrm{H}), 2.48(\mathrm{~s}, 1 \mathrm{H}), 2.29(\mathrm{~s}, 3 \mathrm{H}) .{ }^{13} \mathbf{C}$ NMR (100 MHz, CDCl$\left._{3}\right) \delta 145.7,142.8,138.2,136.2,134.7,132.2,131.7,130.8,129.1,128.6,128.4,128.0$, 127.8, 124.1, 120.4, 73.5, 21.5. HRMS (ESI) $\mathrm{m} / \mathrm{z}$ : $[\mathrm{M}+\mathrm{Na}]^{+}$calcd for (mass of corresponding oxidized product using PDC) $\mathrm{C}_{20} \mathrm{H}_{15} \mathrm{BrNaOS}$ 404.9919; found 404.9921. FTIR (cm $\left.{ }^{-1}\right) 3564$, 3420, 3057, 1609, 1564, 1468, 1437, 1384, 1089, 1007.

\section{(3-Bromophenyl)(2-((4-bromophenyl)thio)phenyl)methanol (4i)}

Following the general procedure, treatment of 3-bromobenzaldehyde 1i (0.046 g, $0.25 \mathrm{mmol})$, 2-(trimethylsilyl)phenyl trifluoromethane sulfonate $2 \mathrm{a}(0.149 \mathrm{~g}, 121 \mu \mathrm{L}, 0.5 \mathrm{mmol})$ and 4bromobenzenethiol 3a $(0.095 \mathrm{~g}, 0.5 \mathrm{mmol})$ in the presence of KF $(0.073 \mathrm{~g}, 1.25 \mathrm{mmol})$ and 18 - 
crown-6 (0.330 g, $1.25 \mathrm{mmol})$ in THF $(1.0 \mathrm{~mL})$ at $25^{\circ} \mathrm{C}$ for $12 \mathrm{~h}$ followed by purification via<smiles>OC(c1cccc(Br)c1)c1ccccc1Sc1ccc(Br)cc1</smiles>

$4 \mathrm{i}$

silica gel flash column chromatography $($ Pet. ether $/$ EtOAc $=93 / 7)$ of the crude reaction mixture afforded (3-bromophenyl)(2-((4bromophenyl)thio)phenyl)methanol $\mathbf{4 i}$ as a white solid (0.102 $\mathrm{g}, 91 \%$ yield).

$\boldsymbol{R}_{\mathbf{f}}($ Pet. ether $/$ EtOAc $=90 / 10): 0.41 ;{ }^{1} \mathbf{H}$ NMR (400 MHz, $\left.\mathbf{C D C l}_{3}\right) \delta$ $7.58(\mathrm{~d}, J=7.8 \mathrm{~Hz}, 1 \mathrm{H}), 7.44-7.22(\mathrm{~m}, 8 \mathrm{H}), 7.12(\mathrm{t}, J=7.8 \mathrm{~Hz}, 1 \mathrm{H}), 6.95$ $(\mathrm{d}, J=8.5 \mathrm{~Hz}, 2 \mathrm{H}), 6.29(\mathrm{~d}, J=3.2 \mathrm{~Hz}, 1 \mathrm{H}), 2.60(\mathrm{~s}, 1 \mathrm{H}) \cdot{ }^{13} \mathbf{C} \mathbf{N M R}\left(\mathbf{1 0 0} \mathbf{M H z}, \mathbf{C D C l}_{3}\right) \delta$ $145.1,145.0,135.8,135.0,132.3,131.6,130.8,130.7,130.1,130.1,129.3,129.0,128.1,125.6$, 122.7, 120.8, 72.8. HRMS (ESI) $\mathrm{m} / \mathrm{z}:[\mathrm{M}+\mathrm{Na}]^{+}$calcd for (mass of corresponding oxidized product using PDC) $\mathrm{C}_{19} \mathrm{H}_{12} \mathrm{Br}_{2} \mathrm{NaOS}$ 468.8868; found 468.8875. FTIR (cm-1) 3580, 3334, $3058,1567,1470,1432,1178,1089,1007,814$.

\section{(2-((4-Bromophenyl)thio)phenyl)(3-chlorophenyl)methanol (4j)}

Following the general procedure, treatment of 3-chlorobenzaldehyde $\mathbf{1 j}(0.035 \mathrm{~g}, 0.028 \mu \mathrm{L}$, $0.25 \mathrm{mmol}), 2$-(trimethylsilyl)phenyl trifluoromethanesulfonate 2a $(0.149 \mathrm{~g}, 121 \mu \mathrm{L}, 0.5$ $\mathrm{mmol})$ and 4-bromobenzenethiol $\mathbf{3 a}(0.095 \mathrm{~g}, 0.5 \mathrm{mmol})$ in the presence of KF $(0.073 \mathrm{~g}, 1.25$ mmol $)$ and 18 -crown-6 $(0.330 \mathrm{~g}, 1.25 \mathrm{mmol})$ in THF $(1.0 \mathrm{~mL})$ at $25^{\circ} \mathrm{C}$ for $12 \mathrm{~h}$ followed by purification via silica gel flash column chromatography (Pet. ether $/$ EtOAc $=95 / 5)$ of the crude reaction mixture afforded (2-((4-bromophenyl)thio)phenyl)(3-chlorophenyl)methanol $\mathbf{4 j}$ as a colorless sticky oil $(0.067 \mathrm{~g}, 66 \%$ yield)<smiles>OC(c1cccc(Cl)c1)c1ccccc1Sc1ccc(Br)cc1</smiles>

$\left(\mathbf{c m}^{-1}\right)$ 3382, 2364, 1569, 1466, 1384, 1178, 1089, 1009.

$\boldsymbol{R}_{\mathbf{f}}($ Pet. ether $/$ EtOAc $=90 / 10): 0.46 ;{ }^{1} \mathbf{H}$ NMR (400 MHz, $\left.\mathbf{C D C l}_{3}\right) \delta$ 7.60-7.58 (m, 1H), 7.41-7.25 (m, 6H), 7.19-7.18 (m, 3H), 6.97-6.93 (m, 2H), 6.29 (s, 1H), 2.52 (bs, 1H). ${ }^{13} \mathbf{C}$ NMR (100 MHz, CDCl 3$) \delta$ $145.00,144.81,135.75,134.87,134.40,132.29,131.65,130.77$, $129.77,129.26,128.98,128.06,127.75,127.20,125.19,120.55,72.82$

HRMS (ESI) m/z: [M+H $]^{+}$calcd for (mass of corresponding oxidized product using $\mathrm{PDC}$ ) $\mathrm{C}_{19} \mathrm{H}_{13} \mathrm{BrClOS} 402.9554$; found 402.9554. FTIR 
<smiles>O=[N+]([O-])c1cccc(C(O)c2ccccc2Sc2ccc(Br)cc2)c1</smiles>

((4-bromophenyl)thio)phenyl)(3-nitrophenyl)methanol 4k as a white solid (0.095 g, 91\% yield).

$\boldsymbol{R}_{\mathbf{f}}($ Pet. ether $/$ EtOAc $=90 / 10): 0.41 ;{ }^{1} \mathbf{H}$ NMR $\left(400 \mathbf{~ M H z}, \mathbf{C D C l}_{3}\right) \delta 8.17(\mathrm{~s}, 1 \mathrm{H}), 8.03(\mathrm{~d}, J=$ 8.1 Hz, 1H), 7.63-7.57 (m, 2H), 7.42-7.36 (m, 3H), 7.31-7.26 (m, 3H), $6.92(\mathrm{~d}, J=9.0 \mathrm{~Hz}, 2 \mathrm{H})$, 6.40 (s, 1H), 3.25 (s, 1H). ${ }^{13}$ C NMR (100 MHz, CDCl3) $\delta$ 148.2, 144.8, 144.5, 135.4, 135.0, 133.1, 132.3, 131.5, 130.6, 129.5, 129.3, 129.3, 128.0, 122.5, 122.0, 120.6, 72.4. HRMS (ESI) m/z: $[\mathrm{M}+\mathrm{Na}]^{+}$calcd for $\mathrm{C}_{19} \mathrm{H}_{14} \mathrm{BrNO}_{3} \mathrm{SNa}$ 437.9770; found 437.9772. FTIR (cm $\left.{ }^{-1}\right)$ 3564, 3061, 1529, 1470, 1437, 1350, 1185, 1089, 1032, 1007.

\section{(2-((4-Bromophenyl)thio)phenyl)(o-tolyl)methanol (4I) ${ }^{7}$}

Following the general procedure, treatment of 2-methylbenzaldehyde 11 ( $0.03 \mathrm{~g}, 0.25 \mathrm{mmol}$, $30 \mu \mathrm{L})$, 2-(trimethylsilyl)phenyl trifluoromethanesulfonate $2 \mathrm{a}(0.149 \mathrm{~g}, 121 \mu \mathrm{L}, 0.5 \mathrm{mmol})$ and<smiles>Cc1ccccc1C(O)c1ccccc1Sc1ccc(Br)cc1</smiles>
4-bromobenzenethiol 3a $(0.094 \mathrm{~g}, 0.5 \mathrm{mmol})$ in the presence of KF $(0.073 \mathrm{~g}$, $1.25 \mathrm{mmol})$ and 18 -crown-6 (0.330 g, $1.25 \mathrm{mmol})$ in THF $(1.0 \mathrm{~mL})$ at $25{ }^{\circ} \mathrm{C}$ for $12 \mathrm{~h}$ followed by purification via silica gel flash column chromatography (Pet. ether $/ \mathrm{EtOAc}=90 / 10)$ of the crude reaction mixture afforded ((2-)(4bromophenyl)thio)phenyl)(o-tolyl)methanol $\mathbf{4 1}$ as a colorless oil (0.071 g, $74 \%$ yield).

$\boldsymbol{R}_{\mathbf{f}}($ Pet. ether $/$ EtOAc $=90 / 10): 0.44 ;{ }^{1} \mathbf{H}$ NMR (400 MHz, $\left.\mathbf{C D C l}_{3}\right) \delta 7.42\left(\mathrm{dd}, J_{l}=7.7 \mathrm{~Hz}, J_{2}\right.$ $=1.0 \mathrm{~Hz}, 1 \mathrm{H}), 7.37-7.31(\mathrm{~m}, 4 \mathrm{H}), 7.28-7.24(\mathrm{~m}, 2 \mathrm{H}), 7.21-7.12(\mathrm{~m}, 3 \mathrm{H}), 7.03(\mathrm{~d}, J=8.6 \mathrm{~Hz}$, 2H), $6.44(\mathrm{~d}, J=4.2 \mathrm{~Hz}, 1 \mathrm{H}), 2.30(\mathrm{~d}, J=4.5 \mathrm{~Hz}, 1 \mathrm{H}), 2.25(\mathrm{~s}, 3 \mathrm{H}) .{ }^{13} \mathbf{C}$ NMR (100 MHz, CDCl $\left._{3}\right) \delta 144.7,140.5,136.0,135.8,134.4,132.8,132.3,131.3,130.6,128.7,128.7,128.1$, 127.8, 126.8, 126.2, 120.7, 70.5, 19.3. HRMS (ESI) $\mathrm{m} / \mathrm{z}:[\mathrm{M}+\mathrm{Na}]^{+}$calcd for (mass of corresponding oxidized product using PDC) $\mathrm{C}_{20} \mathrm{H}_{15} \mathrm{BrNaOS} 404.9919$; found 404.9920. FTIR $\left(\mathbf{c m}^{-1}\right)$ 3362, 3058, 2921, 2365, 1566, 1466, 1384, 1175, 1007. 
(2-((4-Bromophenyl)thio)phenyl)(2-chlorophenyl)methanol (4m)<smiles>OC(c1ccccc1Cl)c1ccccc1Sc1ccc(Br)cc1</smiles>

Following the general procedure, treatment of 2-chlorobenzaldehyde 1m (0.035 g, $0.25 \mathrm{mmol}), 2$-(trimethylsilyl)phenyl trifluoromethanesulfonate 2a $(0.149 \mathrm{~g}, 121 \mu \mathrm{L}, 0.5 \mathrm{mmol})$ and 4-bromobenzenethiol 3a (0.095 g, 0.5 $\mathrm{mmol})$ in the presence of KF $(0.073 \mathrm{~g}, 1.25 \mathrm{mmol})$ and 18-crown-6 (0.330 $\mathrm{g}, 1.25 \mathrm{mmol})$ in THF $(1.0 \mathrm{~mL})$ at $25{ }^{\circ} \mathrm{C}$ for $12 \mathrm{~h}$ followed by purification via silica gel flash column chromatography $($ Pet. ether $/$ EtOAc $=95 / 5)$ of the crude reaction mixture afforded (2-((4-bromophenyl)thio)phenyl)(2chlorophenyl)methanol $\mathbf{4 m}$ as a colorless sticky oil ( $0.086 \mathrm{~g}, 85 \%$ yield).

$\boldsymbol{R}_{\mathbf{f}}($ Pet. ether $/$ EtOAc $=90 / 10): 0.43 ;{ }^{1} \mathbf{H}$ NMR (400 MHz, $\left.\mathbf{C D C l}_{3}\right) \delta$ 7.41-7.26 (m, 8H), 7.25$7.20(\mathrm{~m}, 2 \mathrm{H}), 7.05-7.02(\mathrm{~m}, 2 \mathrm{H}), 6.64(\mathrm{~d}, J=4.1 \mathrm{~Hz}, 1 \mathrm{H}), 2.61(\mathrm{~d}, J=4.2 \mathrm{~Hz}, 1 \mathrm{H}) .{ }^{13} \mathbf{C} \mathbf{~ N M R}$

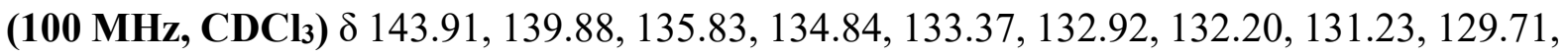
129.05, 128.93, 128.87, 128.71, 128.09, 127.04, 120.57, 70.38. HRMS (ESI) m/z: $[\mathrm{M}+\mathrm{Na}]^{+}$ calcd for (mass of corresponding oxidized product using PDC) $\mathrm{C}_{19} \mathrm{H}_{12} \mathrm{BrClNaOS} 424.9373$; found 424.9379. FTIR ( $\left(\mathbf{c m}^{-1}\right)$ 3579, 3060, 2922, 1925, 1567, 1469, 1483, 1060, 1010.

\section{(2-((4-Bromophenyl)thio)phenyl)(naphthalen-2-yl)methanol (4n)}<smiles>OC(c1ccc2ccccc2c1)c1ccccc1Sc1ccc(Br)cc1</smiles>

Following the general procedure, treatment of 2-naphthaldehyde 1n (0.039 g, $0.25 \mathrm{mmol})$, 2-(trimethylsilyl)phenyl trifluoromethane sulfonate 2a $(0.149 \mathrm{~g}, 121 \mu \mathrm{L}, 0.5 \mathrm{mmol})$ and 4-bromobenzenethiol 3a $(0.095 \mathrm{~g}, 0.5 \mathrm{mmol})$ in the presence of KF $(0.073 \mathrm{~g}, 1.25 \mathrm{mmol})$ and 18-crown-6 (0.330 g, $1.25 \mathrm{mmol})$ in THF $(1.0 \mathrm{~mL})$ at $25{ }^{\circ} \mathrm{C}$ for $12 \mathrm{~h}$ followed by purification via silica gel flash column chromatography $($ Pet. ether $/ \mathrm{EtOAc}=94 / 6)$ of the crude reaction mixture afforded (2((4-bromophenyl)thio)phenyl)(naphthalen-2-yl)methanol $\mathbf{4 n}$ as a white solid $(0.087 \mathrm{~g}, 83 \%$ yield).

$\boldsymbol{R}_{\mathbf{f}}($ Pet. ether $/$ EtOAc $=90 / 10)$ : 0.48; ${ }^{1} \mathbf{H}$ NMR (400 $\left.\mathbf{M H z}, \mathbf{C D C l}_{3}\right) \delta$ 7.81-7.74 (m, 4H), 7.68 (d, $J=7.7 \mathrm{~Hz}, 1 \mathrm{H}), 7.49-7.45(\mathrm{~m}, 2 \mathrm{H}), 7.43-7.37$ (m, 3H), 7.30-7.26 (m, 3H), 6.96 (d, $J=8.5$ $\mathrm{Hz}, 2 \mathrm{H}), 6.51(\mathrm{~d}, J=2.7 \mathrm{~Hz}, 1 \mathrm{H}), 2.68(\mathrm{~d}, J=3.4 \mathrm{~Hz}, 1 \mathrm{H}) .{ }^{13} \mathbf{C} \mathbf{N M R}\left(\mathbf{1 0 0} \mathbf{M H z}, \mathbf{C D C l}_{3}\right) \delta$ $145.5,140.1,136.0,134.8,133.3,132.9,132.2,131.8,130.8,129.1,128.8,128.3,128.2$, 127.7 , 126.3, 126.2, 125.9, 125.0, 120.4, 73.6. HRMS (ESI) m/z: $[\mathrm{M}+\mathrm{Na}]^{+}$calcd for (mass of corresponding oxidized product using PDC) $\mathrm{C}_{23} \mathrm{H}_{15} \mathrm{BrNaOS} 440.9919$; found 440.9930. FTIR $\left(\mathbf{c m}^{-1}\right)$ 3578, 3383, 3056, 3013, 1507, 1469, 1437, 1386, 1089, 1008. 
(2-((4-Bromophenyl)thio)phenyl)(3,5-dibromophenyl)methanol (4o)<smiles>O=C(O)C(c1cc(Br)cc(Br)c1)c1ccccc1Sc1ccc(Br)cc1</smiles>

Following the general procedure, treatment of 3,5-dibromobenzaldehyde 1a (0.066 g, $0.25 \mathrm{mmol}), 2$-(trimethylsilyl)phenyl trifluoromethane sulfonate 2a $(0.149 \mathrm{~g}, 121 \mu \mathrm{L}, 0.5 \mathrm{mmol})$ and 4-bromobenzenethiol 3a $(0.095 \mathrm{~g}, 0.5 \mathrm{mmol})$ in the presence of KF $(0.073 \mathrm{~g}, 1.25 \mathrm{mmol})$ and 18 crown-6 (0.330 g, $1.25 \mathrm{mmol})$ in THF $(1.0 \mathrm{~mL})$ at $25{ }^{\circ} \mathrm{C}$ for $12 \mathrm{~h}$ followed by purification via silica gel flash column chromatography (Pet. ether $/$ EtOAc $=95 / 5)$ of the crude reaction mixture afforded (2-((4bromophenyl)thio)phenyl)(3,5-dibromophenyl)methanol 4a as a colorless sticky oil (0.065 g, $49 \%$ yield).

$\boldsymbol{R}_{\mathbf{f}}($ Pet. ether $/$ EtOAc $=90 / 10)$ : 0.50; ${ }^{1} \mathbf{H}$ NMR (400 MHz, $\left.\mathbf{C D C l}_{3}\right) \delta 7.55(\mathrm{~d}, J=7.6 \mathrm{~Hz}, 1 \mathrm{H})$, $7.48(\mathrm{~s}, 1 \mathrm{H}), 7.44-7.40(\mathrm{~m}, 2 \mathrm{H}), 7.34-7.29(\mathrm{~m}, 5 \mathrm{H}), 6.93-6.91(\mathrm{~m}, 2 \mathrm{H}), 6.27(\mathrm{~d}, J=4.2 \mathrm{~Hz}$, 1H), $2.56(\mathrm{~d}, J=4.4 \mathrm{~Hz}, 1 \mathrm{H}) .{ }^{13} \mathbf{C}$ NMR (100 MHz, CDCl 3$) \delta 146.65,144.68,135.61,135.53$, 133.14, 132.36, 131.41, 130.48, 129.68, 129.33, 128.86, 128.32, 123.08, 120.68, 72.41. HRMS (ESI) $\mathrm{m} / \mathrm{z}:[\mathrm{M}+\mathrm{H}]^{+}$calcd for (mass of corresponding oxidized product using PDC) $\mathrm{C}_{19} \mathrm{H}_{12} \mathrm{Br}_{3} \mathrm{OS}$ 524.8153; found 524.8160. FTIR (cm $\left.{ }^{-1}\right)$ 3405, 3055, 2366, 1584, 1555, 1469 , 1420, 1088, 1009.

\section{(2-((4-Bromophenyl)thio)phenyl)(pyren-1-yl)methanol (4p)}<smiles>OC(c1ccccc1Sc1ccc(Br)cc1)c1ccc2ccc3cccc4ccc1c2c34</smiles>

Following the general procedure, treatment of pyrene-1-carbaldehyde $1 p \quad(0.057 \quad \mathrm{~g}, \quad 0.25 \mathrm{mmol}), \quad$ 2-(trimethylsilyl)phenyl trifluoromethanesulfonate $2 \mathrm{a}(0.149 \mathrm{~g}, 121 \mu \mathrm{L}, 0.5 \mathrm{mmol})$ and 4 bromobenzenethiol 3a $(0.095 \mathrm{~g}, 0.5 \mathrm{mmol})$ in the presence of $\mathrm{KF}$ (0.073 g, $1.25 \mathrm{mmol})$ and 18-crown-6 (0.330 g, $1.25 \mathrm{mmol})$ in THF $(1.0 \mathrm{~mL})$ at $25^{\circ} \mathrm{C}$ for $12 \mathrm{~h}$ followed by purification via silica gel flash column chromatography $($ Pet. ether $/$ EtOAc $=95 / 5)$ of the crude reaction mixture afforded (2-((4-bromophenyl)thio)phenyl)(pyren-1-yl)methanol $\mathbf{4 p}$ as a white solid $(0.098 \mathrm{~g}, 79 \%$ yield $)$.

$\boldsymbol{R}_{\mathbf{f}}($ Pet. ether $/$ EtOAc $=90 / 10)$ : 0.48; ${ }^{1} \mathbf{H}$ NMR (400 MHz, $\left.\mathbf{C D C l}_{3}\right) \delta$ 8.19-8.14 (m, 3H), 8.05$7.97(\mathrm{~m}, 5 \mathrm{H}), 7.93$ (d, $J=8.0 \mathrm{~Hz}, 1 \mathrm{H}), 7.51-7.49$ (m, 1H), 7.44-7.42 (m, 1H), 7.33-7.26 (m, $3 \mathrm{H}), 7.14(\mathrm{~d}, J=8.4 \mathrm{~Hz}, 2 \mathrm{H}), 6.89(\mathrm{~d}, J=8.4 \mathrm{~Hz}, 2 \mathrm{H}), 2.83(\mathrm{~d}, J=4.1 \mathrm{~Hz}, 1 \mathrm{H}) .{ }^{13} \mathbf{C} \mathbf{~ N M R}$ (100 MHz, CDCl3) $\delta$ 145.46, 135.56, 135.47, 134.90, 132.33, 131.94, 131.39, 131.05, 130.82, $130.67,128.97,128.72,128.58,128.30,127.92,127.64,127.46,126.03,125.47,125.26$, 
124.90, 124.84, 124.76, 122.89, 120.37, 70.36. HRMS (ESI) m/z: $[\mathrm{M}+\mathrm{Na}]^{+}$calcd for (mass of corresponding oxidized product using PDC) $\mathrm{C}_{29} \mathrm{H}_{17} \mathrm{BrNaOS} 515.0076$; found 515.0083. FTIR $\left(\mathbf{c m}^{-1}\right)$ 3555, 3287, 3407, 1593, 1468, 1437, 1216, 1009, 845, 757.

\section{(2-((4-Bromophenyl)thio)phenyl)(thiophen-3-yl)methanol (4q)}<smiles>OC(c1ccsc1)c1ccccc1Sc1ccc(Br)cc1</smiles>

Following the general procedure, treatment of thiophene-3-carbaldehyde 1q (0.028 g, $0.2 \mathrm{mmol}), 2$-(trimethylsilyl)phenyl trifluoromethanesulfonate $\mathbf{2 a}$ $(0.149 \mathrm{~g}, 121 \mu \mathrm{L}, 0.5 \mathrm{mmol})$ and 4-bromobenzenethiol 3a (0.095 g, 0.5 $\mathrm{mmol})$ in the presence of KF $(0.073 \mathrm{~g}, 1.25 \mathrm{mmol})$ and 18-crown-6 $(0.330 \mathrm{~g}$, $1.25 \mathrm{mmol})$ in THF $(1.0 \mathrm{~mL})$ at $25^{\circ} \mathrm{C}$ for $12 \mathrm{~h}$ followed by purification via silica gel flash column chromatography $($ Pet. ether $/$ EtOAc $=94 / 6)$ of the crude reaction mixture afforded (2-((4-bromophenyl)thio)phenyl)(thiophen-

3-yl)methanol $\mathbf{4 q}$ as a white solid ( $0.67 \mathrm{~g}, 71 \%$ yield).

$\boldsymbol{R}_{\mathbf{f}}($ Pet. ether $/$ EtOAc $=90 / 10): 0.47 ;{ }^{1} \mathbf{H}$ NMR $\left(400 \mathbf{~ M H z}, \mathbf{C D C l}_{3}\right) \delta$ 7.66-7.64 (m, 1H), 7.41$7.37(\mathrm{~m}, 1 \mathrm{H}), 7.35-7.32(\mathrm{~m}, 3 \mathrm{H}), 7.28-7.26(\mathrm{~m}, 1 \mathrm{H}), 7.25-7.23(\mathrm{~m}, 1 \mathrm{H}), 7.11-7.10(\mathrm{~m}, 1 \mathrm{H})$, 7.00-6.96 (m, 3H), $6.38(\mathrm{~d}, J=4.0 \mathrm{~Hz}, 1 \mathrm{H}), 2.52(\mathrm{~d}, J=4.3 \mathrm{~Hz}, 1 \mathrm{H}) .{ }^{13} \mathbf{C}$ NMR (100 MHz, $\left.\mathbf{C D C l}_{3}\right) \delta 145.3,144.4,136.0,134.6,132.3,131.8,131.0,129.1,128.8,127.7,126.5,126.3$, 122.5, 120.6, 70.2. HRMS (ESI) $\mathrm{m} / \mathrm{z}$ : $[\mathrm{M}+\mathrm{K}]^{+}$calcd for $\mathrm{C}_{17} \mathrm{H}_{13} \mathrm{BrKO}_{2} \mathrm{~S}_{2}$ 414.9223; found 414.9252. FTIR (cm $\left.{ }^{-1}\right)$ 3580, 3470, 3056, 1469, 1438, 1385, 1147, 1087, 1008, 814.

\section{(2-((4-Bromophenyl)thio)phenyl)(thiophen-2-yl)methanol (4r)}

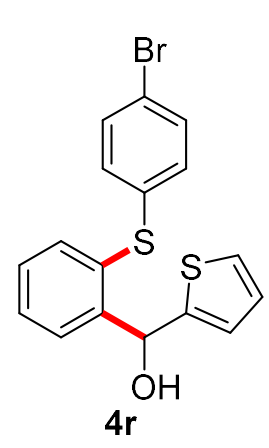

Following the general procedure, treatment of thiophene-2-carbaldehyde 1r (0.028 g, $0.2 \mathrm{mmol}), 2$-(trimethylsilyl)phenyl trifluoromethanesulfonate 2a $(0.149 \mathrm{~g}, 121 \mu \mathrm{L}, 0.5 \mathrm{mmol})$ and 4-bromobenzenethiol 3a $(0.095 \mathrm{~g}, 0.5$ $\mathrm{mmol})$ in the presence of KF $(0.073 \mathrm{~g}, 1.25 \mathrm{mmol})$ and 18-crown-6 $(0.330 \mathrm{~g}$, $1.25 \mathrm{mmol})$ in THF $(1.0 \mathrm{~mL})$ at $25^{\circ} \mathrm{C}$ for $12 \mathrm{~h}$ followed by purification via silica gel flash column chromatography $($ Pet. ether $/$ EtOAc $=94 / 6)$ of the crude reaction mixture afforded (2-((4-bromophenyl)thio)phenyl)(thiophen2-yl)methanol 4r as a white solid (0.69 g, 73\% yield).

$\boldsymbol{R}_{\mathbf{f}}\left(\right.$ Pet. ether $/$ EtOAc $=$ 90/10): 0.47; ${ }^{1} \mathbf{H}$ NMR (400 MHz, $\left.\mathbf{C D C l}_{3}\right) \delta 7.75(\mathrm{dd}, J 1=7.8 \mathrm{~Hz}, J 2$ $=1.0 \mathrm{~Hz}, 1 \mathrm{H}), 7.43-7.39(\mathrm{~m}, 1 \mathrm{H}), 7.36-7.22(\mathrm{~m}, 5 \mathrm{H}), 7.00-6.98(\mathrm{~m}, 2 \mathrm{H}), 6.88(\mathrm{dd}, J 1=5.0 \mathrm{~Hz}$, $J 2=3.6 \mathrm{~Hz}, 1 \mathrm{H}), 6.80-6.79(\mathrm{~m}, 1 \mathrm{H}), 6.53(\mathrm{~d}, J=4.4 \mathrm{~Hz}, 1 \mathrm{H}), 2.79(\mathrm{~d}, J=4.6 \mathrm{~Hz}, 1 \mathrm{H}) .{ }^{13} \mathbf{C}$ NMR (100 MHz, CDCl $) \delta 147.2,145.1,135.9,134.6,132.2,131.7,131.0,129.1,129.0$, 
127.5, 126.8, 125.7, 125.5, 120.5, 69.8. HRMS (ESI) m/z: $[\mathrm{M}+\mathrm{Na}]^{+}$calcd for (mass of corresponding oxidized product using PDC) $\mathrm{C}_{17} \mathrm{H}_{11} \mathrm{BrNaO}_{2} \mathrm{~S} 396.9327$; found 396.9323. FTIR $\left(\mathbf{c m}^{-1}\right)$ 3392, 3060, 2920, 1708, 1564, 1468, 1436, 1385.

\section{Benzofuran-2-yl(2-((4-bromophenyl)thio)phenyl)methanol (4s)}

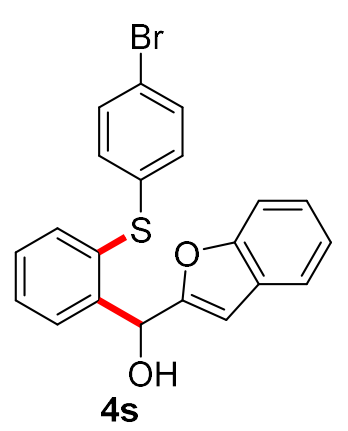

Following the general procedure, treatment of benzofuran-2carbaldehyde $1 \mathrm{~s}$ (0.036 g, $0.25 \mathrm{mmol})$, 2-(trimethylsilyl)phenyl trifluoromethanesulfonate $\mathbf{2 a}(0.149 \mathrm{~g}, 121 \mu \mathrm{L}, 0.5 \mathrm{mmol})$ and 4bromobenzenethiol 3a $(0.095 \mathrm{~g}, 0.5 \mathrm{mmol})$ in the presence of KF $(0.073$ $\mathrm{g}, 1.25 \mathrm{mmol})$ and 18 -crown-6 (0.330 g, $1.25 \mathrm{mmol})$ in THF $(1.0 \mathrm{~mL})$ at $25{ }^{\circ} \mathrm{C}$ for $12 \mathrm{~h}$ followed by purification via silica gel flash column chromatography $($ Pet. ether $/ \mathrm{EtOAc}=94 / 6)$ of the crude reaction mixture afforded benzofuran-2-yl(2-((4-bromophenyl)thio)phenyl)methanol 4s as a white solid (0.086 $\mathrm{g}, 84 \%$ yield).

$\boldsymbol{R}_{\mathbf{f}}($ Pet. ether $/$ EtOAc $=90 / 10): 0.48 ;{ }^{1} \mathbf{H}$ NMR (400 MHz, $\left.\mathbf{C D C l}_{3}\right) \delta 7.75(\mathrm{~d}, J=7.4 \mathrm{~Hz}, 1 \mathrm{H})$, 7.47-7.40 (m, 4H), 7.35-7.24 (m, 4H), 7.20 (t, $J=7.3 \mathrm{~Hz}, 1 \mathrm{H}), 7.00$ (d, $J=8.5 \mathrm{~Hz}, 2 \mathrm{H}), 6.49$ (s, 1H), 6.38 (d, $J=0.6 \mathrm{~Hz}, 1 \mathrm{H}), 2.94(\mathrm{~s}, 1 \mathrm{H}) .{ }^{13} \mathbf{C}$ NMR (100 MHz, CDCl 3$) \delta 157.7,155.1$, $142.4,135.8,134.8,132.2$, 132.1, 130.9, 129.3, 129.2, 128.3, 128.0, 124.5, 123.0, 121.3, 120.6, 111.4, 104.8, 68.1. HRMS (ESI) m/z: $[\mathrm{M}+\mathrm{Na}]^{+}$calcd for (mass of corresponding oxidized product using PDC) $\mathrm{C}_{21} \mathrm{H}_{13} \mathrm{BrNaO}_{2} \mathrm{~S}$ 430.9712; found 430.9718. FTIR (cm ${ }^{-1}$ ) 3552, 3384, $3060,2365,1563,1473,1447,1254,1089,1008$.

\section{Benzo $[b]$ thiophen-3-yl(2-((4-bromophenyl)thio)phenyl)methanol (4t)}

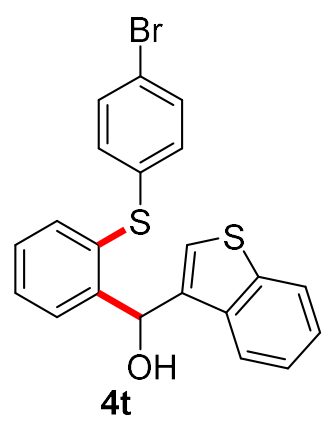

Following the general procedure, treatment of benzo $[b]$ thiophene-3carbaldehyde $1 \mathrm{t} \quad(0.040 \mathrm{~g}, \quad 0.25 \mathrm{mmol}), \quad$ 2-(trimethylsilyl)phenyl trifluoromethanesulfonate $\mathbf{2 a}(0.149 \mathrm{~g}, 121 \mu \mathrm{L}, 0.5 \mathrm{mmol})$ and 4bromobenzenethiol 3a $(0.095 \mathrm{~g}, 0.5 \mathrm{mmol})$ in the presence of KF $(0.073$ $\mathrm{g}, 1.25 \mathrm{mmol})$ and 18 -crown-6 (0.330 g, $1.25 \mathrm{mmol})$ in THF $(1.0 \mathrm{~mL})$ at $25{ }^{\circ} \mathrm{C}$ for $12 \mathrm{~h}$ followed by purification via silica gel flash column chromatography (Pet. ether $/$ EtOAc $=94 / 6)$ of the crude reaction mixture afforded benzo $[b]$ thiophen-3-yl(2-((4-bromophenyl)thio)phenyl)methanol $\mathbf{4 t}$ as a white solid (0.047 g, 44\% yield). 
$\boldsymbol{R}_{\mathbf{f}}($ Pet. ether $/$ EtOAc $=90 / 10): 0.48 ;{ }^{1} \mathbf{H}$ NMR (400 $\left.\mathbf{M H z}, \mathbf{C D C l}_{3}\right) \delta$ 7.84-7.82 (m, $\left.1 \mathrm{H}\right), 7.72-$ $7.70(\mathrm{~m}, 1 \mathrm{H}), 7.58(\mathrm{dd}, J 1=7.7 \mathrm{~Hz}, J 2=1.3 \mathrm{~Hz}, 1 \mathrm{H}), 7.41-7.26(\mathrm{~m}, 7 \mathrm{H}), 7.15(\mathrm{~s}, 1 \mathrm{H}), 7.01(\mathrm{~d}$, $J=8.5 \mathrm{~Hz}, 2 \mathrm{H}), 6.68(\mathrm{~d}, J=4.5 \mathrm{~Hz}, 1 \mathrm{H}), 2.53$ (d, $J=4.6 \mathrm{~Hz}, 1 \mathrm{H}) .{ }^{13} \mathbf{C}$ NMR (100 MHz, CDCl$\left._{3}\right) \delta 144.1,141.0,137.9,137.5,135.7,134.8,132.5,132.2,131.3,129.1,129.1,128.2$, 124.9, 124.6, 124.3, 123.0 122.5, 120.7, 69.1. HRMS (ESI) m/z: [M+Na] $]^{+}$calcd for (mass of corresponding oxidized product using PDC) $\mathrm{C}_{21} \mathrm{H}_{13} \mathrm{BrNaOS}_{2} 446.9483$; found 446.9488. FTIR (cm $\left.{ }^{-1}\right)$ 3677, 3649, 3057, 2365, 1944, 1771, 1549, 1465.

\section{(E)-1-(2-((4-Bromophenyl)thio)phenyl)-3-phenylprop-2-en-1-ol (4u)}

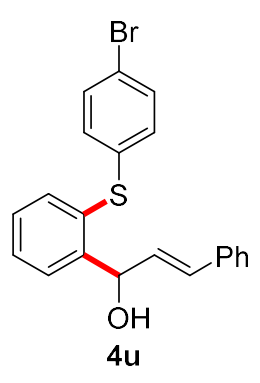

Following the general procedure, treatment of cinnamaldehyde $1 \mathbf{u}(0.033 \mathrm{~g}$, $0.25 \mathrm{mmol}, 31 \mu \mathrm{L}$ ), 2-(trimethylsilyl)phenyl trifluoromethanesulfonate $\mathbf{2 a}$ (0.149 g, $121 \mu \mathrm{L}, 0.5 \mathrm{mmol})$ and 4-bromobenzenethiol 3a (0.094 g, $0.5 \mathrm{mmol})$ in the presence of KF $(0.073 \mathrm{~g}, 1.25 \mathrm{mmol})$ and 18-crown-6 $(0.330 \mathrm{~g}, 1.25$ $\mathrm{mmol})$ in THF $(1.0 \mathrm{~mL})$ at $25^{\circ} \mathrm{C}$ for $12 \mathrm{~h}$ followed by purification via silica gel flash column chromatography (Pet. ether $/$ EtOAc $=90 / 10)$ of the crude reaction mixture afforded (E)-1-(2-((4-bromophenyl)thio)phenyl)-3-phenylprop-2-en-1-ol 4u as a colorless sticky liquid $(0.058 \mathrm{~g}, 58 \%$ yield $)$.

$\boldsymbol{R}_{\mathbf{f}}($ Pet. ether $/$ EtOAc $=90 / 10): 0.40 ;{ }^{1} \mathbf{H}$ NMR (400 MHz, $\left.\mathbf{C D C l}_{3}\right) \delta 7.68\left(\mathrm{dd}, J_{1}=7.6 \mathrm{~Hz}, J_{2}\right.$ $=0.8 \mathrm{~Hz}, 1 \mathrm{H}), 7.43-7.20(\mathrm{~m}, 10 \mathrm{H}), 7.04(\mathrm{~d}, J=8.5 \mathrm{~Hz}, 1 \mathrm{H}), 6.57(\mathrm{~d}, J=15.9 \mathrm{~Hz}, 1 \mathrm{H}), 6.27$ $\left(\mathrm{dd}, J_{1}=15.9 \mathrm{~Hz}, J_{2}=6.2 \mathrm{~Hz}, 1 \mathrm{H}\right), 5.94-5.91(\mathrm{~m}, 1 \mathrm{H}), 2.31(\mathrm{~d}, J=3.7 \mathrm{~Hz}, 1 \mathrm{H}) .{ }^{13} \mathbf{C} \mathbf{N M R}(\mathbf{1 0 0}$ MHz, CDCl $) \delta 145.1,136.6,136.3,134.9,132.4,131.4,131.0,130.8,130.7,129.4,128.8$, 128.7, 127.9, 127.8, 126.7, 120.5, 72.2. HRMS (ESI) m/z: $[\mathrm{M}+\mathrm{H}]^{+}$calcd for (mass of corresponding oxidized product using PDC) $\mathrm{C}_{21} \mathrm{H}_{16} \mathrm{BrOS}$ 395.0100; found 395.0103. FTIR (cm-1) 3057, 2924, 1646, 1566, 1467, 1385, 1061, 1006, 814, 757.

\section{1-(2-((4-Bromophenyl)thio)phenyl)-3-phenylpropan-1-ol (4v)}

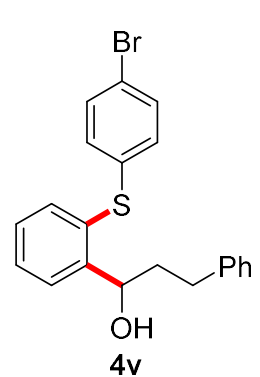

Following the general procedure, treatment of 3-phenylpropanal $1 \mathbf{v}(0.033 \mathrm{~g}$, $0.25 \mathrm{mmol}, 33 \mu \mathrm{L}), 2$-(trimethylsilyl)phenyl trifluoromethanesulfonate $\mathbf{2 a}$ $(0.149 \mathrm{~g}, 121 \mu \mathrm{L}, 0.5 \mathrm{mmol})$ and 4-bromobenzenethiol 3a (0.094 g, $0.5 \mathrm{mmol})$ in the presence of KF $(0.073 \mathrm{~g}, 1.25 \mathrm{mmol})$ and 18-crown-6 (0.330 g, 1.25 $\mathrm{mmol})$ in THF $(1.0 \mathrm{~mL})$ at $25{ }^{\circ} \mathrm{C}$ for $12 \mathrm{~h}$ followed by purification via silica gel flash column chromatography $($ Pet. ether $/$ EtOAc $=90 / 10)$ of the crude 
reaction mixture afforded 1-(2-((4-bromophenyl)thio)phenyl)-3-phenylpropan-1-ol $4 \mathbf{v}$ as a colorless oil ( $0.062 \mathrm{~g}, 62 \%$ yield $)$.

$\boldsymbol{R}_{\mathbf{f}}($ Pet. ether $/$ EtOAc $=90 / 10): 0.43 ;{ }^{1} \mathbf{H}$ NMR (400 MHz, $\left.\mathbf{C D C l}_{3}\right) \delta$ 7.65-7.63 (m, 1H), 7.417.36 (m, 4H), 7.29-7.13 (m, 6H), 7.00 (d, $J=8.6 \mathrm{~Hz}, 2 \mathrm{H}), 5.24-5.21(\mathrm{~m}, 1 \mathrm{H}), 2.86-2.79$ (m, 1H), 2.71-2.63 (m, 1H), $2.14(\mathrm{~d}, J=3.3 \mathrm{~Hz}, 1 \mathrm{H}), 2.06-1.91(\mathrm{~m}, 2 \mathrm{H}) .{ }^{13} \mathbf{C}$ NMR (100 MHz, $\left.\mathbf{C D C l}_{3}\right) \delta 146.9,141.7,136.3,134.7,132.3,130.8,130.8,129.2,128.6,128.5,128.4,127.0$, 126.0, 120.4, 71.0, 40.1, 32.5. HRMS (ESI) m/z: $[\mathrm{M}+\mathrm{Na}]^{+}$calcd for (mass of corresponding oxidized product using PDC) $\mathrm{C}_{21} \mathrm{H}_{17} \mathrm{BrNaOS}$ 419.0076; found 419.0083. FTIR (cm $\left.{ }^{-1}\right) 3385$, 3059, 3025, 2921, 2854, 2365, 1568, 1469, 1385, 1063, 1005.

\section{(2-((4-Bromophenyl)thio)phenyl)(cyclohexyl)methanol (4w)}

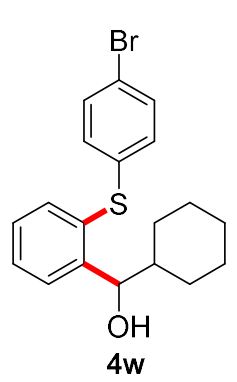

Following the general procedure, treatment of cyclohexanecarbaldehyde $\mathbf{1 w}$ (0.028 g, $0.25 \mathrm{mmol}, 30 \mu \mathrm{L})$, 2-(trimethylsilyl)phenyl trifluoromethane sulfonate 2a (0.149 g, $121 \mu \mathrm{L}, 0.5 \mathrm{mmol})$ and 4-bromobenzenethiol 3a $(0.094$ $\mathrm{g}, 0.5 \mathrm{mmol})$ in the presence of $\mathrm{KF}(0.073 \mathrm{~g}, 1.25 \mathrm{mmol})$ and 18-crown-6 (0.330 $\mathrm{g}, 1.25 \mathrm{mmol})$ in THF $(1.0 \mathrm{~mL})$ at $25^{\circ} \mathrm{C}$ for $12 \mathrm{~h}$ followed by purification via silica gel flash column chromatography (Pet. ether $/$ EtOAc $=90 / 10)$ of the crude reaction mixture afforded (2-((4-bromophenyl)thio)phenyl)(cyclohexyl)methanol $\mathbf{4 w}$ as a colorless oil ( $0.076 \mathrm{~g}, 81 \%$ yield $)$.

$\boldsymbol{R}_{\mathbf{f}}($ Pet. ether /EtOAc $=90 / 10): 0.49 ;{ }^{1} \mathbf{H}$ NMR (400 MHz, $\left.\mathbf{C D C l}_{3}\right) \delta 7.53(\mathrm{~d}, J=7.6 \mathrm{~Hz}, 1 \mathrm{H})$, 7.38-7.19 (m, 5H), $7.05(\mathrm{~d}, J=8.4 \mathrm{~Hz}, 2 \mathrm{H}), 4.96\left(\mathrm{dd}, J_{1}=6.0 \mathrm{~Hz}, J_{2}=3.1 \mathrm{~Hz}, 1 \mathrm{H}\right), 2.03(\mathrm{~d}, J$ $=3.1 \mathrm{~Hz}, 1 \mathrm{H}), 1.90(\mathrm{~d}, J=10.2 \mathrm{~Hz}, 1 \mathrm{H}), 1.74-1.63(\mathrm{~m}, 4 \mathrm{H}), 1.37(\mathrm{~d}, J=9.6 \mathrm{~Hz}, 1 \mathrm{H}), 1.21-$ $1.04(\mathrm{~m}, 5 \mathrm{H}) .{ }^{13} \mathbf{C}$ NMR (100 MHz, $\left.\mathbf{C D C l}_{3}\right) \delta$ 145.7, 136.5, 134.2, 132.3, 131.8, 131.0, 128.6, 128.2 127.9, 120.4 76.0, 44.7, 29.8, 28.1, 26.5, 26.4, 26.1. HRMS (ESI) m/z: $[\mathrm{M}+\mathrm{H}]^{+}$calcd for (mass of corresponding oxidized product using PDC) $\mathrm{C}_{19} \mathrm{H}_{20} \mathrm{BrOS}$ 375.0413; found 375.0413. FTIR (cm-1) 3414, 2926, 2851, 2364, 1567, 1470, 1385, 1085, 1007.

\section{1-(2-((4-Bromophenyl)thio)phenyl)-2-methylpropan-1-ol (4x)}

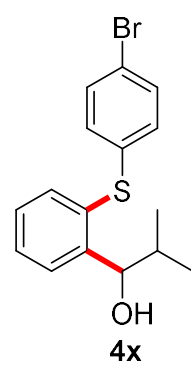

Following the general procedure, treatment of isobutyraldehyde $\mathbf{1 x}(0.018 \mathrm{~g}, 0.25$ mmol, $23 \mu \mathrm{L}), 2$-(trimethylsilyl)phenyl trifluoromethanesulfonate 2a $(0.149 \mathrm{~g}$, $121 \mu \mathrm{L}, 0.5 \mathrm{mmol})$ and 4-bromobenzenethiol 3a $(0.094 \mathrm{~g}, 0.5 \mathrm{mmol})$ in the presence of KF (0.073 g, $1.25 \mathrm{mmol})$ and 18-crown-6 $(0.330 \mathrm{~g}, 1.25 \mathrm{mmol})$ in THF $(1.0 \mathrm{~mL})$ at $25{ }^{\circ} \mathrm{C}$ for $12 \mathrm{~h}$ followed by purification via silica gel flash 
column chromatography $($ Pet. ether $/$ EtOAc $=90 / 10)$ of the crude reaction mixture afforded 1(2-((4-bromophenyl)thio)phenyl)-2-methylpropan-1-ol $\mathbf{4 x}$ as a colorless oil (0.033 g, 39\% yield).

$\boldsymbol{R}_{\mathbf{f}}($ Pet. ether $/$ EtOAc $=90 / 10): 0.41 ;{ }^{1} \mathbf{H}$ NMR (400 MHz, CDCl $) \delta 7.55\left(\mathrm{dd}, J_{1}=7.7 \mathrm{~Hz}, J_{2}\right.$ $=0.8 \mathrm{~Hz}, 1 \mathrm{H}), 7.38-7.29(\mathrm{~m}, 4 \mathrm{H}), 7.23-7.19(\mathrm{~m}, 1 \mathrm{H}), 7.06(\mathrm{~d}, J=8.5 \mathrm{~Hz}, 2 \mathrm{H}), 4.95\left(\mathrm{dd}, J_{1}=\right.$ $\left.6.0 \mathrm{~Hz}, J_{2}=3.8 \mathrm{~Hz}, 1 \mathrm{H}\right), 2.12-2.08(\mathrm{~m}, 1 \mathrm{H}), 2.08-1.96(\mathrm{~m}, 1 \mathrm{H}), 0.98(\mathrm{~d}, J=6.7 \mathrm{~Hz}, 3 \mathrm{H}), 0.87$ $(\mathrm{d}, J=6.8 \mathrm{~Hz}, 3 \mathrm{H}) .{ }^{13} \mathbf{C}$ NMR (100 MHz, $\left.\mathbf{C D C l} 3\right) \delta 145.7,136.3,134.0,132.3,131.7,131.0$, 128.6, 128.2, 127.7, 120.4, 76.4, 34.8, 19.7, 17.4. HRMS (ESI) m/z: $[\mathrm{M}+\mathrm{Na}]^{+}$calcd for (mass of corresponding oxidized product using PDC) $\mathrm{C}_{16} \mathrm{H}_{15} \mathrm{BrNaOS}$ 356.9919; found 356.9922 . FTIR (cm-1) 3419, 2926, 2851, 1664, 1470, 1385, 1088, 1062, 1007.

\section{1,4-Phenylenebis((2-((4-bromophenyl)thio)phenyl)methanol) (4y)}

Following the general procedure, treatment of terephthalaldehyde $1 \mathbf{y}(0.035 \mathrm{~g}, 0.25 \mathrm{mmol}), 2-$<smiles>OC(c1ccc(Br)cc1)c1ccccc1Sc1ccc(Br)cc1</smiles>
(trimethylsilyl)phenyl trifluoro methanesulfonate 2a (0.298 g, 242 $\mu \mathrm{L}, 1.0 \mathrm{mmol})$ and 4-bromobenzenethiol 3a $(0.189 \mathrm{~g}, 1.0 \mathrm{mmol})$ in the presence of KF $(0.146 \mathrm{~g}, 2.5 \mathrm{mmol})$ and 18-crown-6 (0.660 g, $2.5 \mathrm{mmol})$ in $\operatorname{THF}(2.0 \mathrm{~mL})$ at $25^{\circ} \mathrm{C}$ for $12 \mathrm{~h}$ followed by purification via silica gel flash column chromatography (Pet. ether $/$ EtOAc $=75 / 25)$ of the crude reaction mixture afforded 1,4phenylenebis((2-((4-bromophenyl)thio) phenyl) methanol) $\mathbf{4 y}$ as a white solid (83 g, 50\% yield).

$\boldsymbol{R}_{\mathbf{f}}($ Pet. ether $/$ EtOAc $=90 / 10): 0.25 ;{ }^{1} \mathbf{H}$ NMR (400 MHz, $\left.\mathbf{C D C l}_{3}\right) \delta 7.60(\mathrm{~d}, J=6.8 \mathrm{~Hz}, 2 \mathrm{H})$, 7.37-7.28 (m, 8H), 7.25-7.21 (m, 6H), $6.93(\mathrm{dd}, J 1=8.5 \mathrm{~Hz}, J 2=1.4 \mathrm{~Hz}, 4 \mathrm{H}), 6.27(\mathrm{~d}, J=2.8$ $\mathrm{Hz}, 2 \mathrm{H}), 2.61$ (d, $J=3.0 \mathrm{~Hz}, 2 \mathrm{H}) .{ }^{13} \mathbf{C}$ NMR (100 MHz, CDCl$) \delta 145.39,145.36,142.20$, $142.17,136.01,134.54,134.50,132.20,131.74,131.69$, 130.99, 130.94, 129.35, 128.99, 128.96, 128.68, 127.85, 127.84, 127.17, 127.16, 126.48, 120.38, 120.36, 73.08. HRMS (ESI) $\mathrm{m} / \mathrm{z}:[\mathrm{M}+\mathrm{Na}]^{+}$calcd for (mass of corresponding oxidized product using PDC) $\mathrm{C}_{32} \mathrm{H}_{20} \mathrm{Br}_{2} \mathrm{NaO}_{2} \mathrm{~S}_{2}$ 680.9164; found 680.9161. FTIR (cm $\left.{ }^{-1}\right)$ 3583, 3418, 3058, 3011, 2899, $1565,1469,1384,1218,1088$. 


\section{(4-Chlorophenyl)(2-(p-tolylthio)phenyl)methanol (4z)}

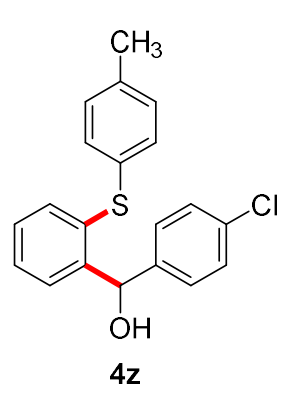

Following the general procedure, treatment of 4-chlorobenzaldehyde 1a (0.035 g, $0.25 \mathrm{mmol}), 2$-(trimethylsilyl)phenyl trifluoromethanesulfonate 2a $(0.149 \mathrm{~g}, 121 \mu \mathrm{L}, 0.5 \mathrm{mmol})$ and 4-methylbenzenethiol 3b $(0.062 \mathrm{~g}, 0.5$ $\mathrm{mmol})$ in the presence of KF $(0.073 \mathrm{~g}, 1.25 \mathrm{mmol})$ and 18-crown-6 $(0.330$ $\mathrm{g}, 1.25 \mathrm{mmol})$ in THF $(1.0 \mathrm{~mL})$ at $25{ }^{\circ} \mathrm{C}$ for $12 \mathrm{~h}$ followed by purification via silica gel flash column chromatography $($ Pet. ether $/$ EtOAc $=90 / 10)$ of the crude reaction mixture afforded (4-chlorophenyl)(2-(p-tolylthio)phenyl)methanol $\mathbf{4 z}$ as a white sticky solid $(0.078 \mathrm{~g}, 91 \%$ yield $)$.

$\boldsymbol{R}_{\mathbf{f}}($ Pet. ether $/$ EtOAc $=90 / 10): 0.41 ;{ }^{1} \mathbf{H}$ NMR $\left(\mathbf{4 0 0} \mathbf{~ M H z}, \mathbf{C D C l}_{3}\right) \delta 7.47(\mathrm{~d}, J=7.5 \mathrm{~Hz}, 1 \mathrm{H})$, 7.28-7.16 (m, 7H), 7.11-7.05 (m, 4H), 6.30 (s, 1H), 2.67 (s, 1H), 2.31 (s, 3H). ${ }^{13}$ C NMR (100 MHz, CDCl3) $\delta$ 144.0, 141.5, 137.4, 134.0, 133.3, 133.0, 131.9, 131.1, 130.2, 128.6, 128.6, 128.4, 128.0, 127.7, 72.6, 21.2. HRMS (ESI) m/z: $[\mathrm{M}+\mathrm{Na}]^{+}$calcd for (mass of corresponding oxidized product using PDC) $\mathrm{C}_{20} \mathrm{H}_{15} \mathrm{ClNaOS}$ 361.0424; found 361.0428. FTIR (cm $\left.{ }^{-1}\right)$ 3384, 3060, 2919, 1900, 1593, 1490, 1179, 1089, 1013, 757.

\section{(4-Chlorophenyl)(2-(phenylthio)phenyl)methanol (4aa)}

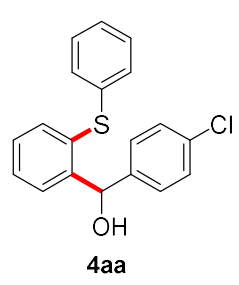

Following the general procedure, treatment of 4-chlorobenzaldehyde 1a $(0.035$ g, $0.25 \mathrm{mmol})$, 2-(trimethylsilyl)phenyl trifluoromethanesulfonate $2 \mathbf{a}(0.149 \mathrm{~g}$, $121 \mu \mathrm{L}, 0.5 \mathrm{mmol})$ and benzenethiol $3 \mathrm{c}(0.055 \mathrm{~g}, 51 \mu \mathrm{L}, 0.5 \mathrm{mmol})$ in the presence of KF $(0.073 \mathrm{~g}, 1.25 \mathrm{mmol})$ and 18 -crown-6 $(0.330 \mathrm{~g}, 1.25 \mathrm{mmol})$ in

THF $(1.0 \mathrm{~mL})$ at $25^{\circ} \mathrm{C}$ for $12 \mathrm{~h}$ followed by purification via silica gel flash column chromatography (Pet. ether $/$ EtOAc $=90 / 10)$ of the crude reaction mixture (4chlorophenyl)(2-(phenylthio)phenyl)methanol 4aa as a light brown sticky oil $(0.077 \mathrm{~g}, 94 \%$ yield).

$\boldsymbol{R}_{\mathbf{f}}($ Pet. ether $/$ EtOAc $=90 / 10): 0.51 ;{ }^{1} \mathbf{H}$ NMR $\left(400\right.$ MHz, $\left.\mathbf{C D C l}_{3}\right) \delta$ 7.52-7.50 (m, 1H), 7.33$7.30(\mathrm{~m}, 2 \mathrm{H}), 7.27-7.13(\mathrm{~m}, 10 \mathrm{H}), 6.30$ (s, 1H), 2.66 (s, 1H). ${ }^{13} \mathbf{C}$ NMR (100 MHz, CDCl3) $\delta$ $144.9,141.4,136.3,134.3,133.3,132.7,129.9,129.3,128.7,128.7,128.6,128.4,127.8,126.8$, 72.7. HRMS (ESI) $\mathrm{m} / \mathrm{z}$ : $[\mathrm{M}+\mathrm{Na}]^{+}$calcd for (mass of corresponding oxidized product using PDC) $\mathrm{C}_{19} \mathrm{H}_{13} \mathrm{ClNaOS}$ 347.0268; found 347.0269. FTIR (cm-1) 3362, 3057, 2920, 2366, 1581, 1484, 1437, 1089, 1017. 
(4-Chlorophenyl)(2-((4-chlorophenyl)thio)phenyl)methanol (4ab)

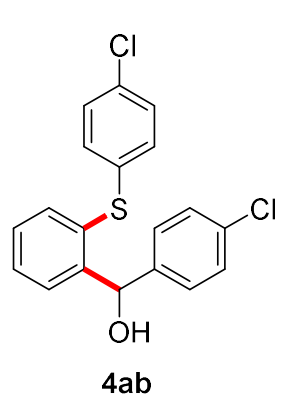

Following the general procedure, treatment of 4-chlorobenzaldehyde 1a (0.035 g, $0.25 \mathrm{mmol}), 2$-(trimethylsilyl)phenyl trifluoromethanesulfonate 2a $(0.149 \mathrm{~g}, 121 \mu \mathrm{L}, 0.5 \mathrm{mmol})$ and 4-chlorobenzenethiol 3d (0.072 g, 0.5 $\mathrm{mmol})$ in the presence of KF $(0.073 \mathrm{~g}, 1.25 \mathrm{mmol})$ and 18-crown-6 $(0.330$ $\mathrm{g}, 1.25 \mathrm{mmol})$ in THF $(1.0 \mathrm{~mL})$ at $25^{\circ} \mathrm{C}$ for $12 \mathrm{~h}$ followed by purification via silica gel flash column chromatography $($ Pet. ether $/$ EtOAc $=90 / 10)$ of the crude reaction mixture afforded (4-chlorophenyl)(2-((4-chlorophenyl)thio)phenyl) methanol 4ab as a light yellow sticky oil (0.073 g, 81\% yield).

$\boldsymbol{R}_{\mathbf{f}}($ Pet. ether $/$ EtOAc $=90 / 10): 0.46 ;{ }^{1} \mathbf{H}$ NMR (400 MHz, $\left.\mathbf{C D C l}_{3}\right) \delta 7.58(\mathrm{~d}, J=7.8 \mathrm{~Hz}, 1 \mathrm{H})$, $7.37\left(\mathrm{td}, J_{1}=7.6 \mathrm{~Hz}, J_{2}=1.1 \mathrm{~Hz}, 1 \mathrm{H}\right), 7.33-7.31(\mathrm{~m}, 1 \mathrm{H}), 7.28-7.18(\mathrm{~m}, 7 \mathrm{H}), 7.06-7.04(\mathrm{~m}$, 2H), $6.30(\mathrm{~d}, J=3.7 \mathrm{~Hz}, 1 \mathrm{H}), 2.55(\mathrm{~d}, J=3.7 \mathrm{~Hz}, 1 \mathrm{H}) .{ }^{13} \mathbf{C}$ NMR (100 MHz, CDCl$) \delta 145.0$, $141.3,134.9,134.4,133.5,132.9,132.1,130.9,129.5,129.0,128.9,128.7,128.5,127.9,72.8$. HRMS (ESI) m/z: [M+Na $]^{+}$calcd for (mass of corresponding oxidized product using PDC) $\mathrm{C}_{19} \mathrm{H}_{12} \mathrm{Cl}_{2} \mathrm{NaOS} 380.9878$; found 380.9885. FTIR ( $\left.\mathbf{c m}^{-1}\right)$ 3418, 3081, 1645, 1469, 1411, 1179 , $1091,1012$.

\section{(4-Chlorophenyl)(2-((4-fluorophenyl)thio)phenyl)methanol (4ac)}

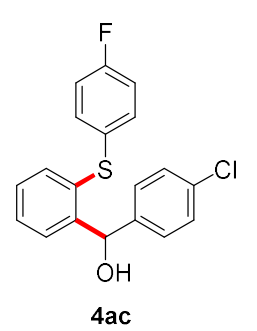

Following the general procedure, treatment of 4-chlorobenzaldehyde 1a $(0.035$ g, $0.25 \mathrm{mmol}), 2$-(trimethylsilyl)phenyl trifluoromethanesulfonate $2 \mathbf{a}(0.149 \mathrm{~g}$, $121 \mu \mathrm{L}, 0.5 \mathrm{mmol})$ and 4-fluorobenzenethiol $3 \mathrm{e}(0.064 \mathrm{~g}, 53 \mu \mathrm{L}, 0.5 \mathrm{mmol})$ in the presence of KF $(0.073 \mathrm{~g}, 1.25 \mathrm{mmol})$ and 18-crown-6 (0.330 g, $1.25 \mathrm{mmol})$ in THF $(1.0 \mathrm{~mL})$ at $25^{\circ} \mathrm{C}$ for $12 \mathrm{~h}$ followed by purification via silica gel flash column chromatography (Pet. ether $/ \mathrm{EtOAc}=90 / 10)$ of the crude reaction mixture afforded (4chlorophenyl)(2-((4-fluorophenyl)thio)phenyl)methanol 4ac as a colorless sticky liquid (0.059 $\mathrm{g}, 68 \%$ yield).

$\boldsymbol{R}_{\mathbf{f}}($ Pet. ether $/ \mathrm{EtOAc}=90 / 10): 0.44 ;{ }^{1} \mathbf{H}$ NMR (400 MHz, CDCl $) \delta 7.53(\mathrm{~d}, J=7.7 \mathrm{~Hz}, 1 \mathrm{H})$, 7.32-7.16 (m, 9H), 6.96 (t, $J=8.5 \mathrm{~Hz}, 2 \mathrm{H}), 6.30(\mathrm{~s}, 1 \mathrm{H}), 2.60(\mathrm{~s}, 1 \mathrm{H}) .{ }^{13} \mathrm{C}$ NMR (100 MHz, CDCl $\left._{3}\right) \delta 162.3(\mathrm{C}-\mathrm{F}, 1 \mathrm{JC}-\mathrm{F}=247.6 \mathrm{~Hz}), 143.9,141.4,133.7,133.5,133.0,132.9,130.6(\mathrm{C}-$ F, 3JC-F = 3.2 Hz), 128.8, 128.7, 128.5, 128.2, 127.7, $116.6(\mathrm{C}-\mathrm{F}, 2 \mathrm{JC}-\mathrm{F}=22.0 \mathrm{~Hz}), 72.7$. HRMS (ESI) m/z: [M+Na $]^{+}$calcd for (mass of corresponding oxidized product using PDC) $\mathrm{C}_{19} \mathrm{H}_{12} \mathrm{ClFNaOS}$ 365.0174; found 365.0184. FTIR (cm $\left.{ }^{-1}\right)$ 3393, 2923, 1896, 1589, 1488, 1227 , 1013,828 . 


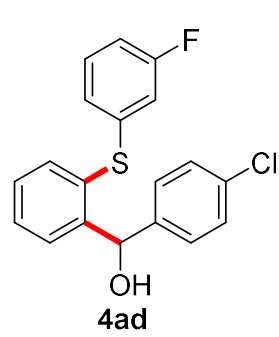

Following the general procedure, treatment of 4-chlorobenzaldehyde 1a (0.035 g, 0.25 mmol), 2-(trimethylsilyl)phenyl trifluoromethanesulfonate 2a $(0.149 \mathrm{~g}, 121 \mu \mathrm{L}, 0.5 \mathrm{mmol})$ and 3-fluorobenzenethiol 3f $(0.064 \mathrm{~g}, 42 \mu \mathrm{L}$, $0.5 \mathrm{mmol})$ in the presence of KF $(0.073 \mathrm{~g}, 1.25 \mathrm{mmol})$ and 18-crown-6 $(0.330 \mathrm{~g}, 1.25 \mathrm{mmol})$ in THF $(1.0 \mathrm{~mL})$ at $25{ }^{\circ} \mathrm{C}$ for $12 \mathrm{~h}$ followed by purification via silica gel flash column chromatography (Pet. ether $/$ EtOAc $=90 / 10)$ of the crude reaction mixture afforded (4-chlorophenyl)(2-((3-fluorophenyl)thio)phenyl)methanol 4ad as a colorless liquid ( $0.066 \mathrm{~g}, 77 \%$ yield).

$\boldsymbol{R}_{\mathbf{f}}($ Pet. ether $/$ EtOAc $=90 / 10): 0.42 ;{ }^{1} \mathbf{H}$ NMR (400 MHz, $\left.\mathbf{C D C l}_{3}\right) \delta 7.62\left(\mathrm{dd}, J_{1}=7.9 \mathrm{~Hz}, J_{2}\right.$ $=1.1 \mathrm{~Hz}, 1 \mathrm{H}), 7.44-7.41(\mathrm{~m}, 2 \mathrm{H}), 7.32-7.14(\mathrm{~m}, 6 \mathrm{H}), 6.86-6.82(\mathrm{~m}, 2 \mathrm{H}), 6.71\left(\mathrm{dt}, J_{1}=9.3 \mathrm{~Hz}\right.$, $\left.J_{2}=2.0 \mathrm{~Hz}, 1 \mathrm{H}\right), 6.30(\mathrm{~s}, 1 \mathrm{H}), 2.56(\mathrm{~s}, 1 \mathrm{H}) .{ }^{13} \mathbf{C}$ NMR (100 MHz, CDCl $) \delta 163.1$ (C-F, 1JC$\mathrm{F}=248.7 \mathrm{~Hz}), 145.9,141.3,139.4(\mathrm{C}-\mathrm{F}, 3 \mathrm{JC}-\mathrm{F}=7.7 \mathrm{~Hz}), 135.7,133.5,130.8,130.5(\mathrm{C}-\mathrm{F}$, $3 \mathrm{JC}-\mathrm{F}=8.5 \mathrm{~Hz}), 129.7,129.0,128.6,128.5,128.0,124.1(\mathrm{C}-\mathrm{F}, 4 \mathrm{JC}-\mathrm{F}=3.0 \mathrm{~Hz}), 115.4(\mathrm{C}-\mathrm{F}$, 2JC-F $=23.7 \mathrm{~Hz}), 113.3(\mathrm{C}-\mathrm{F}, 2 \mathrm{JC}-\mathrm{F}=21.3 \mathrm{~Hz}), 72.9 . \mathbf{H R M S}(\mathbf{E S I}) \mathrm{m} / \mathrm{z}:[\mathrm{M}+\mathrm{Na}]^{+}$calcd for (mass of corresponding oxidized product using PDC) $\mathrm{C}_{19} \mathrm{H}_{12} \mathrm{ClFNaOS}$ 365.0174; found 365.0175. FTIR (cm-1) 3509, 3068, 2924, 2365, 1592, 1481, 1304, 1225, 1150, 1015.

\section{(2-((3-Bromophenyl)thio)phenyl)(4-chlorophenyl)methanol (4ae)}

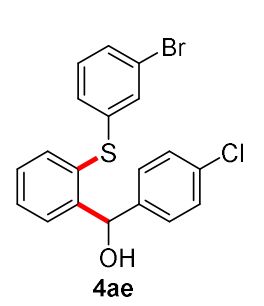

Following the general procedure, treatment of 4-chlorobenzaldehyde 1a (0.035 g, $0.25 \mathrm{mmol}), 2$-(trimethylsilyl)phenyl trifluoromethanesulfonate 2a $(0.149 \mathrm{~g}, 121 \mu \mathrm{L}, 0.5 \mathrm{mmol})$ and 3-bromobenzenethiol 3g $(0.094 \mathrm{~g}, 52 \mu \mathrm{L}, 0.5$ $\mathrm{mmol})$ in the presence of KF $(0.073 \mathrm{~g}, 1.25 \mathrm{mmol})$ and 18-crown-6 $(0.330 \mathrm{~g}$, $1.25 \mathrm{mmol})$ in THF $(1.0 \mathrm{~mL})$ at $25{ }^{\circ} \mathrm{C}$ for $12 \mathrm{~h}$ followed by purification via silica gel flash column chromatography (Pet. ether $/$ EtOAc $=90 / 10)$ of the crude reaction mixture afforded (2-((3-bromophenyl)thio)phenyl)(4-chlorophenyl)methanol 4ae as a colorless oil (0.066 g, 65\% yield).

$\boldsymbol{R}_{\mathbf{f}}($ Pet. ether $/$ EtOAc $=90 / 10): 0.45 ;{ }^{1} \mathbf{H}$ NMR (400 MHz, $\left.\mathbf{C D C l}_{3}\right) \delta 7.63(\mathrm{~d}, J=6.8 \mathrm{~Hz}, 1 \mathrm{H})$, 7.48-7.39 (m, 2H), 7.33-7.20 (m, 6H), 7.12 (t, $J=1.6 \mathrm{~Hz}, 1 \mathrm{H}), 7.05$ (t, $J=7.9 \mathrm{~Hz}, 1 \mathrm{H}), 6.99$ $6.97(\mathrm{~m}, 1 \mathrm{H}), 6.30(\mathrm{~d}, J=3.3 \mathrm{~Hz}, 1 \mathrm{H}), 2.56(\mathrm{~d}, J=3.8 \mathrm{~Hz}, 1 \mathrm{H}) .{ }^{13} \mathbf{C}$ NMR (100 MHz, CDCl $\left.\mathbf{3}\right)$ $\delta 145.8,141.2,139.2,135.6,133.5,131.1,130.8,130.5,129.6,129.4,129.0,128.6,128.5$, 128.0, 127.2, 123.2, 72.9. HRMS (ESI) $\mathrm{m} / \mathrm{z}:[\mathrm{M}+\mathrm{H}]^{+}$calcd for (mass of corresponding 
oxidized product using PDC) $\mathrm{C}_{19} \mathrm{H}_{13} \mathrm{BrClOS}$ 402.9554; found 402.9556. FTIR (cm $\left.{ }^{-1}\right)$ 3355, 3057, 2852, 2365, 1566, 1460, 1403, 1089, 1013.

\section{(4-Chlorophenyl)(2-(o-tolylthio)phenyl)methanol (4af)}

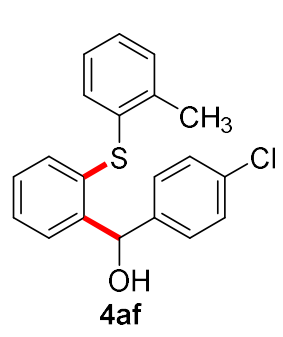

Following the general procedure, treatment of 4-chlorobenzaldehyde 1a (0.035 g, $0.25 \mathrm{mmol}), 2$-(trimethylsilyl)phenyl trifluoromethanesulfonate 2a $(0.149 \mathrm{~g}, 121 \mu \mathrm{L}, 0.5 \mathrm{mmol})$ and 2-methylbenzenethiol 3h (0.062 g, 0.5 $\mathrm{mmol})$ in the presence of KF $(0.073 \mathrm{~g}, 1.25 \mathrm{mmol})$ and 18-crown-6 $(0.330$ $\mathrm{g}, 1.25 \mathrm{mmol})$ in THF $(1.0 \mathrm{~mL})$ at $25{ }^{\circ} \mathrm{C}$ for $12 \mathrm{~h}$ followed by purification via silica gel flash column chromatography $($ Pet. ether $/$ EtOAc $=90 / 10)$ of the crude reaction mixture afforded (4-chlorophenyl)(2-(o-tolylthio)phenyl)methanol 4af as a pale brown sticky liquid (0.077 g, 90\% yield).

$\boldsymbol{R}_{\mathbf{f}}($ Pet. ether $/$ EtOAc $=90 / 10): 0.41 ;{ }^{1} \mathbf{H}$ NMR (400 $\left.\mathbf{M H z}, \mathbf{C D C l}_{3}\right) \delta 7.50(\mathrm{~d}, J=7.4 \mathrm{~Hz}, 1 \mathrm{H})$, 7.28-7.13 (m, 8H), 7.10-6.98 (m, 3H), 6.26 (s, 1H), 2.71 (s, 1H), 2.30 (s, 3H). ${ }^{13}$ C NMR (100 MHz, CDCl $) \delta 143.9,141.3,138.8,134.3,133.3,133.1,132.4,131.5,130.7,128.7,128.5$, 128.4, 127.87, 127.7, 127.5, 126.9, 72.58, 20.6. HRMS (ESI) m/z: $[\mathrm{M}+\mathrm{Na}]^{+}$calcd for (mass of corresponding oxidized product using PDC) $\mathrm{C}_{20} \mathrm{H}_{15} \mathrm{ClNaOS} 361.0424$; found 361.0432 . FTIR (cm $\left.{ }^{-1}\right)$ 3388, 3058, 1589, 1490, 1463, 1090, 1013, 753.

\section{(4-Chlorophenyl)(2-((2-chlorophenyl)thio)phenyl)methanol (4ag)}

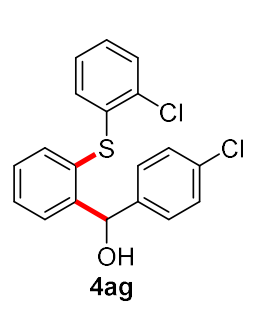

Following the general procedure, treatment of 4-chlorobenzaldehyde 1a (0.035 g, $0.25 \mathrm{mmol})$, 2-(trimethylsilyl)phenyl trifluoromethanesulfonate 2a $(0.149 \mathrm{~g}, 121 \mu \mathrm{L}, 0.5 \mathrm{mmol})$ and 2-chlorobenzenethiol $3 \mathbf{i}(0.072 \mathrm{~g}, 0.5 \mathrm{mmol})$ in the presence of KF $(0.073 \mathrm{~g}, 1.25 \mathrm{mmol})$ and 18-crown-6 $(0.330 \mathrm{~g}, 1.25$ mmol) in THF $(1.0 \mathrm{~mL})$ at $25{ }^{\circ} \mathrm{C}$ for $12 \mathrm{~h}$ followed by purification via silica gel flash column chromatography (Pet. ether $/$ EtOAc $=90 / 10)$ of the crude reaction mixture (4chlorophenyl)(2-((2-chlorophenyl)thio)phenyl)methanol 4ag as a colorless sticky liquid (0.041 g, $45 \%$ yield).

$\boldsymbol{R}_{\mathbf{f}}($ Pet. ether $/$ EtOAc $=90 / 10)$ : 0.44; ${ }^{1} \mathbf{H}$ NMR (400 MHz, $\left.\mathbf{C D C l}_{3}\right) \delta 7.65(\mathrm{~d}, J=7.7 \mathrm{~Hz}, 1 \mathrm{H})$, 7.46-7.29 (m, 4H), 7.24-6.15 (m, 4H), 7.07 (t, $J=7.2 \mathrm{~Hz}, 1 \mathrm{H}), 6.96$ (t, $J=7.4 \mathrm{~Hz}, 1 \mathrm{H}), 6.59$ (d, $J=7.6 \mathrm{~Hz}, 1 \mathrm{H}), 6.29$ (s, 1H), 2.79 (s, 1H). ${ }^{13} \mathbf{C}$ NMR (100 MHz, CDCl $) \delta 146.4,141.2$, $136.3,136.1,133.3,132.4,129.9,129.8,129.7,129.0,129.0,128.5,128.4,128.0,127.2,127.0$, 72.8. HRMS (ESI) $\mathrm{m} / \mathrm{z}$ : $[\mathrm{M}+\mathrm{Na}]^{+}$calcd for (mass of corresponding oxidized product using 
PDC) $\mathrm{C}_{19} \mathrm{H}_{12} \mathrm{Cl}_{2} \mathrm{NaOS} 380.9878$; found 380.9882. FTIR (cm $\left.{ }^{-1}\right)$ 3379, 3058, 2921, 2364, 1570, 1489, 1451, 1089, 1020.

\section{(4-Chlorophenyl)(2-((2-fluorophenyl)thio)phenyl)methanol (4ah)}

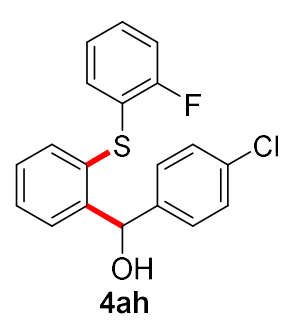

Following the general procedure, treatment of 4-chlorobenzaldehyde 1a (0.035 g, $0.25 \mathrm{mmol}), 2$-(trimethylsilyl)phenyl trifluoromethanesulfonate 2a $(0.149 \mathrm{~g}, 121 \mu \mathrm{L}, 0.5 \mathrm{mmol})$ and 2-fluorobenzenethiol 3j $(0.064 \mathrm{~g}, 53 \mu \mathrm{L}$, $0.5 \mathrm{mmol})$ in the presence of KF $(0.073 \mathrm{~g}, 1.25 \mathrm{mmol})$ and 18-crown-6

$(0.330 \mathrm{~g}, 1.25 \mathrm{mmol})$ in THF $(1.0 \mathrm{~mL})$ at $25{ }^{\circ} \mathrm{C}$ for $12 \mathrm{~h}$ followed by purification via silica gel flash column chromatography (Pet. ether $/$ EtOAc $=90 / 10$ ) of the crude reaction mixture afforded (4-chlorophenyl)(2-((2-fluorophenyl)thio)phenyl)methanol 4ah as a pale brown sticky liquid ( $0.037 \mathrm{~g}, 43 \%$ yield).

$\boldsymbol{R}_{\mathbf{f}}($ Pet. ether $/$ EtOAc $=90 / 10): 0.42 ;{ }^{1} \mathbf{H}$ NMR (400 MHz, $\left.\mathbf{C D C l}_{3}\right) \delta$ 7.57-7.56 (m, 1H), 7.37$7.19(\mathrm{~m}, 8 \mathrm{H}), 7.06(\mathrm{t}, J=8.9 \mathrm{~Hz}, 1 \mathrm{H}), 7.01-6.95(\mathrm{~m}, 2 \mathrm{H}), 6.37(\mathrm{~s}, 1 \mathrm{H}), 2.76(\mathrm{~s}, 1 \mathrm{H}) .{ }^{13} \mathbf{C ~ N M R}$ $\left(\mathbf{1 0 0} \mathrm{MHz}, \mathrm{CDCl}_{3}\right) \delta 160.6(\mathrm{C}-\mathrm{F}, 1 \mathrm{JC}-\mathrm{F}=246.6 \mathrm{~Hz}), 144.9,141.3,134.0,134.3,133.2(\mathrm{C}-\mathrm{F}$, 4JC-F = 1.0 Hz), 131.3, 129.0, 129.0, $128.8(\mathrm{C}-\mathrm{F}, 3 \mathrm{JC}-\mathrm{F}=4.4 \mathrm{~Hz}), 128.6,128.5,127.9,124.8$ $(\mathrm{C}-\mathrm{F}, 3 \mathrm{JC}-\mathrm{F}=3.7 \mathrm{~Hz}), 123.2(\mathrm{C}-\mathrm{F}, 2 \mathrm{JC}-\mathrm{F}=17.4 \mathrm{~Hz}), 116.0(\mathrm{C}-\mathrm{F}, 2 \mathrm{JC}-\mathrm{F}=21.9 \mathrm{~Hz}), 72.7$. HRMS (ESI) m/z: [M+Na $]^{+}$calcd for (mass of corresponding oxidized product using PDC) $\mathrm{C}_{19} \mathrm{H}_{12} \mathrm{ClFNaOS} 365.0174$; found 365.0180. FTIR (cm $\left.{ }^{-1}\right)$ 3384, 3062, 2923, 1900, 1571, 1468, $1222,1092,1015$.

\section{(4-Chlorophenyl)(2-(naphthalen-2-ylthio)phenyl)methanol (4ai)}

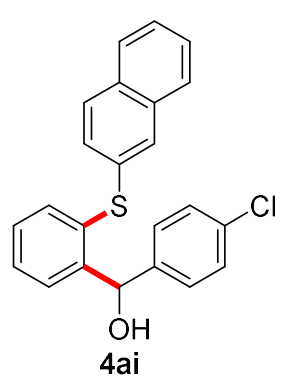

Following the general procedure, treatment of 4-chlorobenzaldehyde 1a (0.035 g, $0.25 \mathrm{mmol}), 2$-(trimethylsilyl)phenyl trifluoromethanesulfonate 2a $(0.149 \mathrm{~g}, 121 \mu \mathrm{L}, 0.5 \mathrm{mmol})$ and naphthalene-2-thiol 3k (0.080 g, $0.5 \mathrm{mmol})$ in the presence of KF $(0.073 \mathrm{~g}, 1.25 \mathrm{mmol})$ and 18-crown-6 (0.330 g, 1.25 $\mathrm{mmol})$ in THF $(1.0 \mathrm{~mL})$ at $25^{\circ} \mathrm{C}$ for $12 \mathrm{~h}$ followed by purification via silica gel flash column chromatography (Pet. ether $/$ EtOAc $=90 / 10)$ of the crude

reaction mixture (4-chlorophenyl)(2-(naphthalen-2-ylthio)phenyl)methanol 4ai as a pale brown sticky liquid ( $0.060 \mathrm{~g}, 64 \%$ yield).

$\boldsymbol{R}_{\mathbf{f}}\left(\right.$ Pet. ether $/$ EtOAc $=$ 90/10): 0.49; ${ }^{1} \mathbf{H}$ NMR (400 MHz, $\left.\mathbf{C D C l}_{3}\right) \delta$ 7.81-7.79 (m, 1H), 7.72 $(\mathrm{d}, J=8.6 \mathrm{~Hz}, 1 \mathrm{H}), 7.68-7.59(\mathrm{~m}, 3 \mathrm{H}), 7.50-7.45(\mathrm{~m}, 2 \mathrm{H}), 7.39-7.35(\mathrm{~m}, 2 \mathrm{H}), 7.31-7.22(\mathrm{~m}$, 6H), 6.38 (s, 1H), 2.71 (s, 1H). ${ }^{13} \mathbf{C}$ NMR (100 MHz, CDCl $) \delta 144.7,141.4,134.1,133.8$, 
$133.4,133.4,132.8,132.2,129.0,128.8,128.7,128.6,128.6,128.5,127.8,127.8,127.7,127.4$, 126.8, 126.3, 72.8. HRMS (ESI) $\mathrm{m} / \mathrm{z}$ : $[\mathrm{M}+\mathrm{Na}]^{+}$calcd for (mass of corresponding oxidized product using PDC) $\mathrm{C}_{23} \mathrm{H}_{15} \mathrm{ClNaOS}$ 397.0424; found 397.0437. FTIR (cm') 3417, 3055, $1625,1588,1492,1180,1090,1014$.

\section{(2-((4-Bromophenyl)thio)-4,5-dimethylphenyl)(4-chlorophenyl)methanol (4aj)}<smiles>Cc1cc(Sc2ccc(Br)cc2)c(C(O)c2ccc(Cl)cc2)cc1C</smiles>

chromatography (Pet. ether $/$ EtOAc $=90 / 10)$ of the crude reaction mixture (2-((4bromophenyl)thio)-4,5-dimethylphenyl)(4-chlorophenyl)methanol 4aj as a brown sticky solid (0.081 g, 75\% yield).

$\boldsymbol{R}_{\mathbf{f}}($ Pet. ether $/$ EtOAc $=90 / 10): 0.52 ;{ }^{1} \mathbf{H}$ NMR (400 MHz, $\left.\mathbf{C D C l}_{3}\right) \delta 7.32(\mathrm{~s}, 1 \mathrm{H}), 7.31-7.27$ (m, 2H), 7.25-7.19 (m, 5H), 6.90-6.87 (m, 2H), 6.24 (d, $J=3.1 \mathrm{~Hz}, 1 \mathrm{H}), 2.48(\mathrm{~d}, J=3.2 \mathrm{~Hz}$, 1H), 2.28 (s, 3H), 2.22 (s, 3H). ${ }^{13} \mathbf{C}$ NMR (100 MHz, $\left.\mathbf{C D C l}_{3}\right) \delta 143.5,141.7,138.9,137.8$, 137.1, 137.0, 133.3, 132.1, 129.5, 129.3, 128.5, 128.2, 127.1, 119.7, 72.7, 19.9, 19.4. HRMS (ESI) $\mathrm{m} / \mathrm{z}$ : $[\mathrm{M}+\mathrm{Na}]^{+}$calcd for (mass of corresponding oxidized product using PDC) $\mathrm{C}_{21} \mathrm{H}_{16} \mathrm{BrClNaOS}$ 452.9686; found 452.9693. FTIR (cm $\left.{ }^{-1}\right)$ 3415, 2918, 2857, 2364, 1598, 1473, 1087, 1007.

\section{(6-((4-Bromophenyl)thio)benzo[d][1,3] dioxol-5-yl)(4-chlorophenyl)methanol (4ak)}

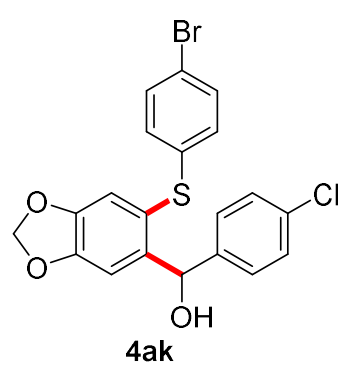

Following the general procedure, treatment of 4-chlorobenzaldehyde 1a (0.035 g, $0.25 \mathrm{mmol}), \quad$ 6-(trimethylsilyl)benzo[d][1,3]dioxol-5-yl

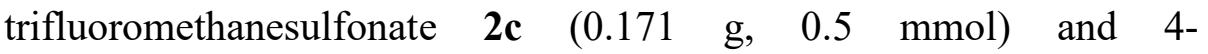
bromobenzenethiol $3 \mathrm{a}(0.094 \mathrm{~g}, 0.5 \mathrm{mmol})$ in the presence of KF $(0.073$ $\mathrm{g}, 1.25 \mathrm{mmol})$ and 18 -crown-6 (0.330 g, $1.25 \mathrm{mmol})$ in THF $(1.0 \mathrm{~mL})$ at $25{ }^{\circ} \mathrm{C}$ for $12 \mathrm{~h}$ followed by purification via silica gel flash column chromatography (Pet. ether $/$ EtOAc $=90 / 10)$ of the crude reaction mixture (6-((4bromophenyl)thio)benzo[d][1,3]dioxol-5-yl)(4-chlorophenyl)methanol 4ak as a brown sticky liquid $(0.069 \mathrm{~g}, 61 \%$ yield $)$. 
$\boldsymbol{R}_{\mathbf{f}}($ Pet. ether $/$ EtOAc $=90 / 10)$ : 0.33; ${ }^{1} \mathbf{H}$ NMR (400 $\left.\mathbf{M H z}, \mathbf{C D C l}_{3}\right) \delta$ 7.32-7.30 (m, 2H), 7.23 (s, 4H), $7.05(\mathrm{~s}, 1 \mathrm{H}), 6.91-6.88(\mathrm{~m}, 3 \mathrm{H}), 6.30(\mathrm{~s}, 1 \mathrm{H}), 6.00-5.98(\mathrm{~m}, 2 \mathrm{H}), 2.37(\mathrm{~s}, 1 \mathrm{H}) .{ }^{13} \mathrm{C}$ NMR (100 MHz, CDCl 3 ) $\delta 149.4,147.9,141.5,141.2,137.1,133.5,132.2,129.3,128.7$, 128.1, 122.4, 119.9, 115.3, 108.2, 102.0, 72.5. HRMS (ESI) m/z: $[\mathrm{M}+\mathrm{Na}]^{+}$calcd for (mass of corresponding oxidized product using PDC) $\mathrm{C}_{20} \mathrm{H}_{12} \mathrm{BrClNaO}_{3} \mathrm{~S} 468.9271$; found 468.9274 . FTIR $\left(\mathbf{c m}^{-1}\right)$ 3588, 2895, 2364, 2333, 1473, 1234, 1038, 1008.

(6-((4-Bromophenyl)thio)-2,3-dihydro-1H-inden-5-yl)(4-chlorophenyl)methanol (4al)

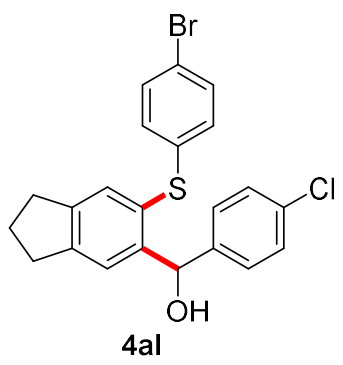

Following the general procedure, treatment of 4-chlorobenzaldehyde 1a (0.035 g, $0.25 \mathrm{mmol})$, 6-(trimethylsilyl)-2,3-dihydro-1H-inden-5-yl trifluoromethanesulfonate $\mathbf{2 d}(0.169 \mathrm{~g}, \quad 0.5 \mathrm{mmol})$ and $4-$ bromobenzenethiol 3a $(0.094 \mathrm{~g}, 0.5 \mathrm{mmol})$ in the presence of $\mathrm{KF}(0.073$ $\mathrm{g}, 1.25 \mathrm{mmol})$ and 18 -crown-6 $(0.330 \mathrm{~g}, 1.25 \mathrm{mmol})$ in THF $(1.0 \mathrm{~mL})$ at $25^{\circ} \mathrm{C}$ for $12 \mathrm{~h}$ followed by purification via silica gel flash column chromatography (Pet. ether $/$ EtOAc $=90 / 10)$ of the crude reaction mixture (6-(4bromophenyl)thio)-2,3-dihydro-1H-inden-5-yl)(4-chlorophenyl)methanol 4al as a brown sticky liquid (0.079 g, 71\% yield).

$\boldsymbol{R}_{\mathbf{f}}($ Pet. ether $/$ EtOAc $=90 / 10)$ : 0.52; ${ }^{1} \mathbf{H}$ NMR (400 MHz, $\left.\mathbf{C D C l}_{3}\right) \delta 7.39(\mathrm{~s}, 1 \mathrm{H}), 7.32-7.21$ (m, 7H), 6.93-6.90 (m, 2H), $6.30(\mathrm{~d}, J=4.0 \mathrm{~Hz}, 1 \mathrm{H}), 2.93-2.85(\mathrm{~m}, 4 \mathrm{H}), 2.46$ (d, $J=4.0 \mathrm{~Hz}$, 1H), 2.08 (quint, $J=7.5 \mathrm{~Hz}, 2 \mathrm{H}) .{ }^{13} \mathbf{C}$ NMR (100 MHz, CDCl $) \delta 146.7,145.6,144.0,141.8$, 137.2 , 133.3, 132.2, 131.7, 129.7, 128.6, 128.3, 128.1, 124.0, 119.8, 72.9, 33.0, 32.4, 25.6. HRMS (ESI) $\mathrm{m} / \mathrm{z}$ : $[\mathrm{M}+\mathrm{H}]^{+}$calcd for (mass of corresponding oxidized product using PDC) $\mathrm{C}_{22} \mathrm{H}_{17} \mathrm{BrClOS}$ 442.9867; found 442.9864. FTIR ( $\left.\mathbf{c m}^{-1}\right)$ 3384, 2955, 2842, 2365, 1568, 1467 , 1386, 1089, 1006.

\section{(2-((4-Bromophenyl)thio)-4,5-difluorophenyl)(4-chlorophenyl)methanol (4am)}<smiles>OC(c1ccc(Cl)cc1)c1cc(F)c(F)cc1Sc1ccc(Br)cc1</smiles>
chromatography (Pet. ether $/$ EtOAc $=90 / 10)$ of the crude reaction mixture (2-((4(0.035 g, $0.25 \mathrm{mmol}), \quad$ 4,5-difluoro-2-(trimethylsilyl)phenyl trifluoromethanesulfonate $\quad \mathbf{2 e} \quad(0.167 \mathrm{~g}, \quad 0.5 \mathrm{mmol})$ and 4bromobenzenethiol 3a $(0.094 \mathrm{~g}, 0.5 \mathrm{mmol})$ in the presence of KF $(0.073$ $\mathrm{g}, 1.25 \mathrm{mmol})$ and 18 -crown-6 (0.330 g, $1.25 \mathrm{mmol})$ in THF $(1.0 \mathrm{~mL})$ at $25{ }^{\circ} \mathrm{C}$ for $12 \mathrm{~h}$ followed by purification via silica gel flash column

Following the general procedure, treatment of 4-chlorobenzaldehyde 1a 
bromophenyl)thio)-4,5-difluorophenyl)(4-chlorophenyl)methanol 4am as a colorless sticky liquid $(0.075 \mathrm{~g}, 68 \%$ yield $)$.

$\boldsymbol{R}_{\mathbf{f}}($ Pet. ether $/$ EtOAc $=90 / 10): 0.49 ;{ }^{1} \mathbf{H}$ NMR (400 MHz, $\left.\mathbf{C D C l}_{3}\right) \delta 7.48\left(\mathrm{dd}, J_{1}=11.3 \mathrm{~Hz}, J_{2}\right.$ $=8.2 \mathrm{~Hz}, 1 \mathrm{H}), 7.39-7.36(\mathrm{~m}, 2 \mathrm{H}), 7.27-7.21(\mathrm{~m}, 4 \mathrm{H}), 7.09\left(\mathrm{dd}, J_{1}=10.1 \mathrm{~Hz}, J_{2}=7.7 \mathrm{~Hz}, 1 \mathrm{H}\right)$, 6.99-6.94 (m, 2H), 6.19 (s, 1H), 2.06 (s, 1H). $\left.{ }^{13} \mathbf{C ~ N M R ~ ( 1 0 0 ~ M H z , ~ C D C l} 3\right) \delta 150.6$ (C-F, 1JC$\mathrm{F}=251.5 \mathrm{~Hz}, \mathrm{C}-\mathrm{F}, 2 \mathrm{JC}-\mathrm{F}=12.3 \mathrm{~Hz}), 149.8(\mathrm{C}-\mathrm{F}, 1 \mathrm{JC}-\mathrm{F}=248.6 \mathrm{~Hz}, \mathrm{C}-\mathrm{F}, 2 \mathrm{JC}-\mathrm{F}=12.7 \mathrm{~Hz})$, 142.0 (unresolved dd), 140.5, 134.0, 132.6, 131.4, 128.9, 128.5, 128.0 (C-F, 3JC-F = 5.6 Hz, $\mathrm{C}-\mathrm{F}, 3 \mathrm{JC}-\mathrm{F}=5.9 \mathrm{~Hz}), 122.5(\mathrm{C}-\mathrm{F}, 2 \mathrm{JC}-\mathrm{F}=18.3 \mathrm{~Hz}), 121.5,116.7(\mathrm{C}-\mathrm{F}, 2 \mathrm{JC}-\mathrm{F}=18.8 \mathrm{~Hz})$, 72.0. HRMS (ESI) $\mathrm{m} / \mathrm{z}$ : $[\mathrm{M}+\mathrm{Na}]^{+}$calcd for (mass of corresponding oxidized product using PDC) $\mathrm{C}_{19} \mathrm{H}_{10} \mathrm{BrClF}_{2} \mathrm{NaOS}$ 460.9185; found 460.9193. FTIR (cm-1) 3322, 2365, 1597, 1492, 1390, 1286, 1133, 1089, 1007.

\section{(2-((4-Bromophenyl)thio)-3,6-dimethylphenyl)(4-chlorophenyl)methanol (4an)}

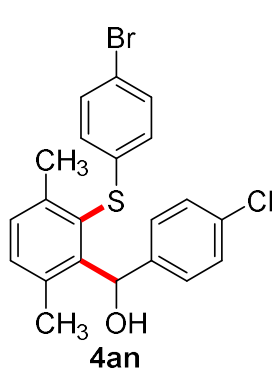

Following the general procedure, treatment of 4-chlorobenzaldehyde 1a (0.035 g, $0.25 \mathrm{mmol}), \quad$ 3,6-dimethyl-2-(trimethylsilyl)phenyl trifluoromethanesulfonate $2 \mathbf{f}(0.163 \mathrm{~g}, 0.5 \mathrm{mmol})$ and 4-bromobenzenethiol 3a $(0.094 \mathrm{~g}, 0.5 \mathrm{mmol})$ in the presence of $\mathrm{KF}(0.073 \mathrm{~g}, 1.25 \mathrm{mmol})$ and 18crown-6 $(0.330 \mathrm{~g}, 1.25 \mathrm{mmol})$ in THF $(1.0 \mathrm{~mL})$ at $25^{\circ} \mathrm{C}$ for $12 \mathrm{~h}$ followed by purification via silica gel flash column chromatography (Pet. ether

/EtOAc $=90 / 10)$ of the crude reaction mixture (2-((4-bromophenyl)thio)-3,6dimethylphenyl)(4-chlorophenyl)methanol 4an as a brown sticky liquid (0.077 g, 71\% yield). $\boldsymbol{R}_{\mathbf{f}}($ Pet. ether $/$ EtOAc $=90 / 10): 0.51 ;{ }^{1} \mathbf{H}$ NMR (400 MHz, $\left.\mathbf{C D C l}_{3}\right) \delta$ 7.27-7.17 (m, 6H), 7.08 (d, $J=8.3 \mathrm{~Hz}, 2 \mathrm{H}), 6.75$ (d, $J=8.5 \mathrm{~Hz}, 2 \mathrm{H}), 6.58$ (d, $J=8.1 \mathrm{~Hz}, 1 \mathrm{H}), 3.45$ (bs, 1H), 2.31 (s, 3H), 2.29 (s, 3H). ${ }^{13} \mathbf{C}$ NMR (100 MHz, $\left.\mathbf{C D C l}_{3}\right) \delta$ 145.4, 142.5, 142.2, 136.5, 136.4, 133.5, 132.5, 132.2, 130.8, 129.7, 128.3, 127.7, 127.1, 119.1, 72.5, 21.5, 20.6. HRMS (ESI) m/z: $[\mathrm{M}+\mathrm{H}]^{+}$calcd for (mass of corresponding oxidized product using $\mathrm{PDC}$ ) $\mathrm{C}_{21} \mathrm{H}_{17} \mathrm{BrClOS}$ 430.9867; found 430.9865. FTIR ( $\left.\mathbf{c m}^{-1}\right)$ 3446, 2922, 2853, 1897, 1591, 1493, 1466, 1382, $1087,1006$.

\section{(3-((4-Bromophenyl)thio)naphthalen-2-yl)(4-chlorophenyl)methanol (4ao)}

Following the general procedure, treatment of 4-chlorobenzaldehyde $1 \mathrm{a}(0.035 \mathrm{~g}, 0.25 \mathrm{mmol})$, 3-(trimethylsilyl)naphthalen-2-yl trifluoromethanesulfonate $2 \mathrm{~g}(0.174 \mathrm{~g}, 0.5 \mathrm{mmol})$ and 4bromobenzenethiol 3a $(0.094 \mathrm{~g}, 0.5 \mathrm{mmol})$ in the presence of KF $(0.073 \mathrm{~g}, 1.25 \mathrm{mmol})$ and 18 - 
crown-6 $(0.330 \mathrm{~g}, 1.25 \mathrm{mmol})$ in THF $(1.0 \mathrm{~mL})$ at $25^{\circ} \mathrm{C}$ for $12 \mathrm{~h}$ followed by purification via silica gel flash column chromatography $($ Pet. ether $/$ EtOAc $=90 / 10)$ of the crude reaction mixture (3-((4-bromophenyl)thio)naphthalen-2-yl)(4-chlorophenyl)methanol $4 \mathbf{a o}$ as a pale brown sticky liquid ( $0.072 \mathrm{~g}, 63 \%$ yield).

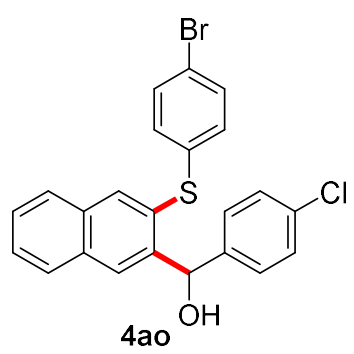

$\boldsymbol{R}_{\mathbf{f}}($ Pet. ether $/$ EtOAc $=90 / 10): 0.45 ;{ }^{1} \mathbf{H}$ NMR (400 MHz, CDCl $) \delta$ $8.06(\mathrm{~s}, 1 \mathrm{H}), 7.90(\mathrm{~s}, 1 \mathrm{H}), 7.86(\mathrm{~d}, J=7.6 \mathrm{~Hz}, 1 \mathrm{H}), 7.74(\mathrm{~d}, J=7.6 \mathrm{~Hz}$, $1 \mathrm{H}), 7.55-7.48(\mathrm{~m}, 2 \mathrm{H}), 7.33-7.22(\mathrm{~m}, 6 \mathrm{H}), 6.95(\mathrm{~d}, J=8.5 \mathrm{~Hz}, 2 \mathrm{H})$, $6.31(\mathrm{~s}, 1 \mathrm{H}), 6.20(\mathrm{bs}, 1 \mathrm{H}) .{ }^{13} \mathbf{C}$ NMR (100 MHz, CDCl $) \delta 141.9$, $141.2,136.1,135.4,133.7,133.3,132.4,130.5,129.3,128.9,128.7$, 128.2, 127.4, 127.4, 127.4, 127.1, 120.5, 73.3. HRMS (ESI) m/z: $[\mathrm{M}+\mathrm{Na}]^{+}$calcd for (mass of corresponding oxidized product using PDC) $\mathrm{C}_{23} \mathrm{H}_{14} \mathrm{BrClNaOS}$ 474.9529; found 474.9536. FTIR (cm-1) 3402, 2923, 2365, 1588, 1467, 1382, 1088, 1007, 898 .

\section{(1-((4-Bromophenyl)thio)naphthalen-2-yl)(4-chlorophenyl)methanol (4ap) and} (2-((4-Bromophenyl)thio)naphthalen-1-yl)(4-chlorophenyl)methanol (4ap')<smiles>OC(c1ccc(Cl)cc1)c1ccc2ccccc2c1Sc1ccc(Br)cc1</smiles><smiles>OC(c1ccc(Cl)cc1)c1c(Sc2ccc(Br)cc2)ccc2ccccc12</smiles>
Following the general procedure, treatment of 4-chlorobenzaldehyde 1a (0.035 g, $0.25 \mathrm{mmol}), \quad 1-$ (trimethylsilyl)naphthalen-2-yl trifluoromethanesulfonate $\mathbf{2 h}(0.174$ $\mathrm{g}, 0.5 \mathrm{mmol})$ and 4-bromobenzenethiol 3a $(0.095 \mathrm{~g}, 0.5 \mathrm{mmol})$ in the presence of KF $(0.073 \mathrm{~g}$, $1.25 \mathrm{mmol})$ and 18 -crown-6 $(0.330 \mathrm{~g}, 1.25 \mathrm{mmol})$ in THF $(1.0 \mathrm{~mL})$ at $25^{\circ} \mathrm{C}$ for $12 \mathrm{~h}$ followed by purification via silica gel flash column chromatography (Pet. ether $/ \mathrm{EtOAc}=90 / 10)$ of the crude reaction mixture afforded (1-((4-bromophenyl)thio)naphthalen-2-yl)(4-chlorophenyl) methanol (4ap) as a white solid (0.043 g, 38\% yield) and (2-((4-bromophenyl)thio)naphthalen1-yl)(4-chlorophenyl)methanol (4ap') as a white solid (0.045 g, 40\% yield) as separable mixture of regioisomers.

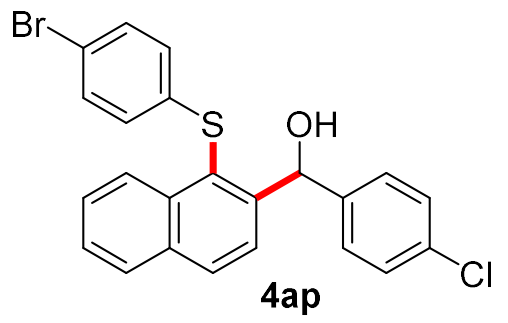

Data of 4ap: $\boldsymbol{R}_{\mathbf{f}}($ Pet. ether $/$ EtOAc $=90 / 10): 0.45 ;{ }^{1} \mathbf{H}$ NMR (400 MHz, $\left.\mathbf{C D C l}_{3}\right) \delta$ 7.45-7.42 (m, 1H), $7.99(\mathrm{~d}, J=8.4 \mathrm{~Hz}$, $1 \mathrm{H}), 7.89-7.86(\mathrm{~m}, 1 \mathrm{H}), 7.77$ (d, $J=8.7 \mathrm{~Hz}, 1 \mathrm{H}), 7.55-7.50$ (m, 2H), 7.28-7.25 (m, 2H), 7.21-7.19 (m, 4H), 6.77 (d, $J=3.2$ $\mathrm{Hz}, 1 \mathrm{H}), 6.71(\mathrm{~d}, J=8.6 \mathrm{~Hz}, 2 \mathrm{H}), 2.55(\mathrm{~d}, J=3.9 \mathrm{~Hz}, 1 \mathrm{H}) .{ }^{13} \mathrm{C}$ NMR (100 MHz, CDCl3) $\delta$ 147.13, 141.64, 137.09, 134.99, 
$133.98,133.39,132.11,131.61,128.72,128.62,128.20,128.01,127.71,126.91,126.68$, 125.68, 125.12, 118.96, 73.43. HRMS (ESI) $\mathrm{m} / \mathrm{z}$ : $[\mathrm{M}+\mathrm{K}]^{+}$calcd for $\mathrm{C}_{23} \mathrm{H}_{16} \mathrm{BrClOSK}$ 492.9425; found 492.9441. FTIR (cm-1) 3359, 3058, 3009, 1772, 1625, 1495, 1468, 1413. CCDC 2040671 (4ap) contains the supplementary crystallographic data for this paper. These data can be obtained free of charge from Data Centre via www.ccdc.cam.ac.uk/data request/cif.

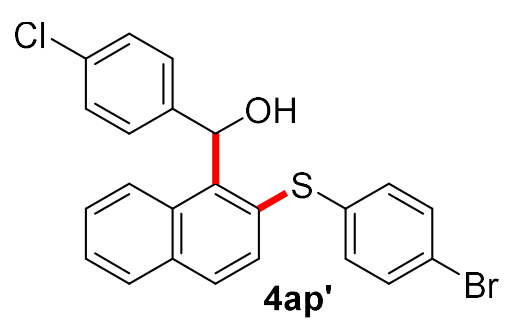

Data of 4ap': $\boldsymbol{R}_{\mathbf{f}}($ Pet. ether $/$ EtOAc $=90 / 10): 0.43 ;{ }^{1} \mathbf{H}$ NMR $\left(400 \mathrm{MHz}, \mathbf{C D C l}_{3}\right) \delta 8.13(\mathrm{~d}, J=8.6 \mathrm{~Hz}, 1 \mathrm{H}), 7.82(\mathrm{~d}, J=8.1$ $\mathrm{Hz}, 1 \mathrm{H}), 7.77$ (d, $J=8.9 \mathrm{~Hz}, 1 \mathrm{H}), 7.48-7.43$ (m, 2H), 7.39-7.37 $(\mathrm{m}, 3 \mathrm{H}), 7.26-7.24(\mathrm{~m}, 4 \mathrm{H}), 7.15-7.10(\mathrm{~m}, 3 \mathrm{H}), 3.16(\mathrm{~d}, J=$ $4.3 \mathrm{~Hz}, 1 \mathrm{H}) .{ }^{13} \mathrm{C}$ NMR (100 MHz, CDCl 3$) \delta 141.84,140.33$, $135.79,134.37,132.78,132.50,131.72,131.41,130.60,130.07,128.81,128.54,127.19$, 126.86, 126.73, 126.37, 120.99, 72.17. HRMS (ESI) m/z: $[\mathrm{M}+\mathrm{K}]^{+}$calcd for $\mathrm{C}_{23} \mathrm{H}_{16} \mathrm{BrClOSK}$ 492.9425; found 492.9438. FTIR (cm $\left.\mathbf{c m}^{-1}\right)$ 3449, 3078, 1897, 1708, 1588, 1495, 1467, 1391.

\section{(2-((4-Bromophenyl)thio)-4-methylphenyl)(4-chlorophenyl)methanol (4aq) and}

\section{( 2-((4-Bromophenyl)thio)-5-methylphenyl)(4-chlorophenyl)methanol (4aq')}

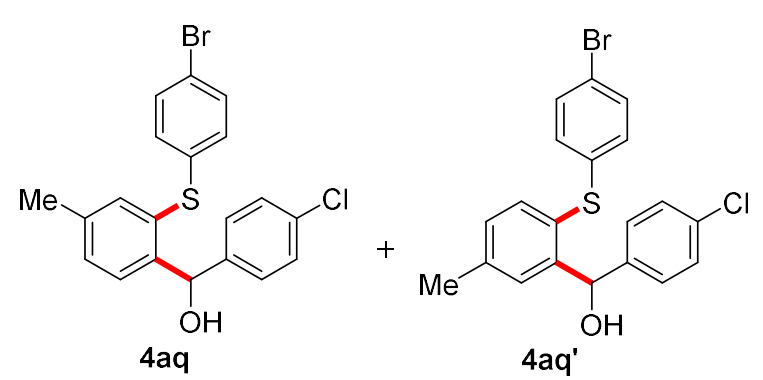

Following the general procedure, treatment of 4-chlorobenzaldehyde $1 \mathbf{a}(0.035 \mathrm{~g}, 0.25 \mathrm{mmol})$, 4-methyl-2-(trimethylsilyl)phenyl trifluoromethanesulfonate $\mathbf{2 i}(0.156 \mathrm{~g}, \quad 0.5$ $\mathrm{mmol})$ and 4-bromobenzenethiol 3a $(0.094 \mathrm{~g}$, $0.5 \mathrm{mmol})$ in the presence of KF $(0.073 \mathrm{~g}, 1.25$ mmol $)$ and 18-crown-6 $(0.330 \mathrm{~g}, 1.25 \mathrm{mmol})$ in THF $(1.0 \mathrm{~mL})$ at $25^{\circ} \mathrm{C}$ for $12 \mathrm{~h}$ followed by purification via silica gel flash column chromatography (Pet. ether $/$ EtOAc $=90 / 10)$ of the crude reaction mixture afforded inseparable (1:1.1) regioisomeric mixture of (2-((4bromophenyl)thio)-4-methylphenyl)(4-chlorophenyl)methanol $\mathbf{4 a q}$ and (2-((4bromophenyl)thio)-5-methylphenyl)(4-chlorophenyl)methanol 4aq' as a colorless sticky liquid (0.093 g, 89\% yield).

$\boldsymbol{R}_{\mathbf{f}}($ Pet. ether $/$ EtOAc $=90 / 10): 0.47 ;{ }^{1} \mathbf{H}$ NMR (400 $\left.\mathbf{~ M H z}, \mathbf{C D C l}_{3}\right)$ of Major isomer $\delta$ 7.45$7.40(\mathrm{~m}, 1 \mathrm{H}), 7.34-7.29(\mathrm{~m}, 3 \mathrm{H}), 7.25-7.19(\mathrm{~m}, 4 \mathrm{H}), 7.10\left(\mathrm{dd}, J_{l}=7.8 \mathrm{~Hz}, J_{2}=1.2 \mathrm{~Hz}, 1 \mathrm{H}\right)$, $6.95(\mathrm{~d}, J=8.5 \mathrm{~Hz}, 2 \mathrm{H}), 6.26$ (s, 1H), 2.49 (bs, 1H), 2.30 (s, 3H). ${ }^{13} \mathbf{C}$ NMR (100 MHz, CDCl $)$ $\delta 142.6,141.6,138.9,136.8,135.5,133.3,132.3,131.1,130.5,129.9,128.7,128.6,128.4$, 
120.3, 72.7, 21.0. Representative peak for minor isomer: $\left.{ }^{1} \mathbf{H} \mathbf{~ N M R ~ ( 4 0 0 ~} \mathbf{~ M H z}, \mathbf{C D C l}_{3}\right) \delta$ $6.89(\mathrm{~d}, J=8.6 \mathrm{~Hz}, 2 \mathrm{H}), 6.27$ (s, 1H), 2.37 (s, 3H). ${ }^{13} \mathbf{C}$ NMR (100 MHz, CDCl 3$) \delta$ 145.7, 141.5, 140.0, 136.2, 135.8, 133.4, 132.2, 130.2, 129.8, 129.6, 128.3, 127.9, 127.4, 119.9, 72.8, 21.5. HRMS (ESI) $\mathrm{m} / \mathrm{z}$ : $[\mathrm{M}+\mathrm{H}]^{+}$calcd for (mass of corresponding oxidized product using PDC) $\mathrm{C}_{20} \mathrm{H}_{15} \mathrm{BrClOS}$ 416.9710; found 416.9715. FTIR (cm $\left.{ }^{-1}\right)$ 3383, 2910, 1895, 1598, 1491, 1469, 1383, 1089, 1008.

(2-((4-Bromophenyl)thio)-4-fluorophenyl)(4-chlorophenyl)methanol (4ar) and (2-((4-Bromophenyl)thio)-5-fluorophenyl)(4-chlorophenyl)methanol (4ar')

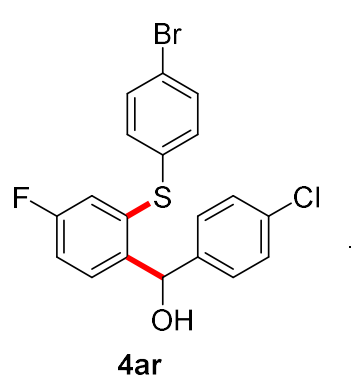

4ar

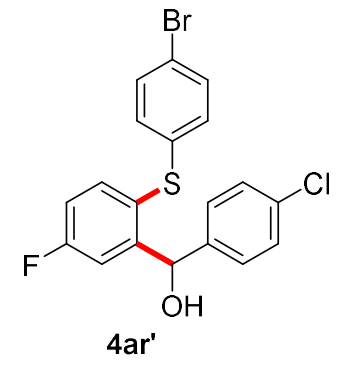

Following the general procedure, treatment of 4chlorobenzaldehyde 1a $(0.035 \mathrm{~g}, 0.25 \mathrm{mmol})$, 4methyl-2-(trimethylsilyl)phenyl trifluoromethanesulfonate $2 \mathbf{i}(0.156 \mathrm{~g}, 0.5 \mathrm{mmol})$ and 4-bromobenzenethiol 3a(0.094 g, $0.5 \mathrm{mmol})$ in the presence of KF $(0.073 \mathrm{~g}, 1.25 \mathrm{mmol})$ and

18-crown-6 $(0.330 \mathrm{~g}, 1.25 \mathrm{mmol})$ in THF $(1.0 \mathrm{~mL})$ at $25^{\circ} \mathrm{C}$ for $12 \mathrm{~h}$ followed by purification via silica gel flash column chromatography $($ Pet. ether $/$ EtOAc $=90 / 10)$ of the crude reaction mixture afforded inseparable (1:1.5) regioisomeric mixture of (2-((4-bromophenyl)thio)-4fluorophenyl)(4-chlorophenyl)methanol 4ar and (2-((4-bromophenyl)thio)-5-fluorophenyl)(4chlorophenyl)methanol 4ar' as a colorless sticky liquid (0.093 g, 88\% yield).

$\boldsymbol{R}_{\mathbf{f}}($ Pet. ether $/$ EtOAc $=90 / 10): 0.47 ;{ }^{1} \mathbf{H}$ NMR (400 $\left.\mathbf{~ M H z , ~} \mathbf{C D C l}_{3}\right)$ of minor isomer $\delta 7.53$ $\left(\mathrm{dd}, J_{l}=8.7 \mathrm{~Hz}, J_{2}=5.9 \mathrm{~Hz}, 1 \mathrm{H}\right), 7.42-7.38(\mathrm{~m}, 2 \mathrm{H}), 7.31-7.22(\mathrm{~m}, 5 \mathrm{H}), 7.02-6.97(\mathrm{~m}, 1 \mathrm{H})$, 6.89-6.84 (m, 2H), 6.23 (s, 1H), 2.52 (s, 1H). ${ }^{13} \mathbf{C ~ N M R ~ ( 1 0 0 ~ M H z , ~ C D C l ~} 3$ ) $\delta 162.3$ (C-F, 1JC$\mathrm{F}=250.5 \mathrm{~Hz}), 141.1,139.6(\mathrm{C}-\mathrm{F}, 3 \mathrm{JC}-\mathrm{F}=3.3 \mathrm{~Hz}), 136.2$, 135.4, $133.7(\mathrm{C}-\mathrm{F}, 3 \mathrm{JC}-\mathrm{F}=10.9$ $\mathrm{Hz}), 132.8,129.9,129.3(\mathrm{C}-\mathrm{F}, 3 \mathrm{JC}-\mathrm{F}=8.4 \mathrm{~Hz}), 128.4,122.1,119.0(\mathrm{C}-\mathrm{F}, 2 \mathrm{JC}-\mathrm{F}=23.5 \mathrm{~Hz})$, $115.3(\mathrm{C}-\mathrm{F}, 2 \mathrm{JC}-\mathrm{F}=21.3 \mathrm{~Hz}), 72.2$. Representative peak for major isomer: ${ }^{1} \mathrm{H}$ NMR (400 MHz, CDCl $) \delta 7.08(\mathrm{~d}, J=8.6 \mathrm{~Hz}, 2 \mathrm{H}) .{ }^{13} \mathrm{C}$ NMR (100 MHz, CDCl 3$) \delta 163.5$ (C-F, 1JC-F $=250.5 \mathrm{~Hz}), 148.6(\mathrm{C}-\mathrm{F}, 3 \mathrm{JC}-\mathrm{F}=7.2 \mathrm{~Hz}), 140.7,137.7(\mathrm{C}-\mathrm{F}, 3 \mathrm{JC}-\mathrm{F}=8.2 \mathrm{~Hz}), 135.3,133.9$, 132.7, 128.8, 128.6, 126.0 (C-F, 3JC-F = 3.2 Hz), 120.3, $116.2(\mathrm{C}-\mathrm{F}, 2 \mathrm{JC}-\mathrm{F}=22.0 \mathrm{~Hz}), 115.1$ (C-F, 2JC-F $=23.3 \mathrm{~Hz}$ ), 72.7. HRMS (ESI) m/z: $[\mathrm{M}+\mathrm{H}]^{+}$calcd for (mass of corresponding oxidized product using PDC) $\mathrm{C}_{19} \mathrm{H}_{12} \mathrm{BrClFOS} 420.9459$; found 420.9460. FTIR (cm-1) 3289, 2923, 2366, 1899, 1599, 1468, 1088, 1009. 


\section{Ethyl 2-(2-((4-bromophenyl)thio)phenyl)-2-hydroxy-2-phenylacetate (6a)}<smiles>CCOC(=O)C(O)(c1ccccc1)c1ccccc1Sc1ccc(Br)cc1</smiles>

$6 a$

Following the general procedure, treatment of ethyl 2-oxo-2-phenylacetate 5a (0.044 g, $0.25 \quad \mathrm{mmol}, \quad 40 \quad \mu \mathrm{L}), \quad$ 2-(trimethylsilyl)phenyl trifluoromethanesulfonate $\mathbf{2 a}(0.149 \mathrm{~g}, 121 \mu \mathrm{L}, 0.5 \mathrm{mmol})$ and 4bromobenzenethiol 3a $(0.94 \mathrm{~g}, 0.5 \mathrm{mmol})$ in the presence of KF $(0.073 \mathrm{~g}, 1.25$ $\mathrm{mmol})$ and 18 -crown-6 $(0.330 \mathrm{~g}, 1.25 \mathrm{mmol})$ in THF $(1.0 \mathrm{~mL})$ at $25^{\circ} \mathrm{C}$ for 12 $\mathrm{h}$ followed by purification via silica gel flash column chromatography (Pet. ether $/$ EtOAc $=90 / 10)$ of the crude reaction mixture afforded ethyl 2-(2-((4bromophenyl)thio)phenyl)-2-hydroxy-2-phenylacetate 6a as a colorless oil (0.078 g, 70\% yield).

$\boldsymbol{R}_{\mathbf{f}}\left(\right.$ Pet. ether $/$ EtOAc $=$ 90/10): 0.4; ${ }^{1} \mathbf{H}$ NMR (400 MHz, $\left.\mathbf{C D C l}_{3}\right) \delta$ 7.71-7.68 (m, 2H), 7.46$7.36(\mathrm{~m}, 6 \mathrm{H}), 7.31-7.01(\mathrm{~m}, 4 \mathrm{H}), 6.81\left(\mathrm{dd}, J_{1}=7.9 \mathrm{~Hz}, J_{2}=1.2 \mathrm{~Hz}, 1 \mathrm{H}\right), 4.36-4.28(\mathrm{~m}, 2 \mathrm{H})$,

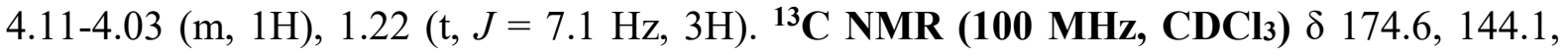
$140.3,137.1,135.7,135.1,132.1,131.5,130.2,129.3,128.5,128.4,127.4,127.1,120.6,81.4$, 63.0, 14.0. HRMS (ESI) m/z: [M+Na] $]^{+}$calcd for $\mathrm{C}_{22} \mathrm{H}_{19} \mathrm{BrNaO}_{3} \mathrm{~S} 465.0130$; found 465.0131 . FTIR (cm-1) 3758, 3469, 3272, 3060, 1729, 1492, 1469, 1239, 1165, 1065, 1008.

\section{1-(2-((4-Bromophenyl)thio)phenyl)-2,2,2-trifluoro-1-phenylethan-1-ol (8a)}

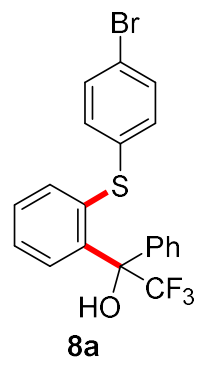

Following the general procedure, treatment of 2,2,2-trifluoro-1-phenylethan-1one $7 \mathbf{a} \quad(0.043 \mathrm{~g}, \quad 0.25 \mathrm{mmol}, \quad 35 \mu \mathrm{L}), \quad$ 2-(trimethylsilyl)phenyl trifluoromethanesulfonate $\mathbf{2 a}(0.149 \mathrm{~g}, \quad 121 \mu \mathrm{L}, 0.5 \mathrm{mmol})$ and 4bromobenzenethiol $3 \mathrm{a}(0.94 \mathrm{~g}, 0.5 \mathrm{mmol})$ in the presence of $\mathrm{KF}(0.073 \mathrm{~g}, 1.25$ mmol) and 18-crown-6 $(0.330 \mathrm{~g}, 1.25 \mathrm{mmol})$ in THF $(1.0 \mathrm{~mL})$ at $25^{\circ} \mathrm{C}$ for $12 \mathrm{~h}$ followed by purification via silica gel flash column chromatography (Pet. ether $/$ EtOAc $=95 / 05)$ of the crude reaction mixture afforded 1-(2-((4-bromophenyl)thio)phenyl)2,2,2-trifluoro-1-phenylethan-1-ol 8a as a colorless oil (0.057 g, 52\% yield).

$\boldsymbol{R}_{\mathbf{f}}\left(\right.$ Pet. ether $/$ EtOAc $=$ 95/05): 0.54; ${ }^{1} \mathbf{H}$ NMR (400 MHz, CDCl $) \delta 7.7(\mathrm{~d}, J=7.9 \mathrm{~Hz}, 1 \mathrm{H})$, 7.38-7.24 (m, 10H), $6.94(\mathrm{~d}, J=8.4 \mathrm{~Hz}, 2 \mathrm{H}), 5.13(\mathrm{~s}, 1 \mathrm{H}) .{ }^{13} \mathbf{C} \mathbf{N M R}\left(\mathbf{1 0 0} \mathbf{~ M H z}, \mathbf{C D C l}_{3}\right) \delta$ 140.2, 139.1, 136.2, 135.0, 134.7, 132.7, 132.5, 129.6, 128.8, 128.2, 127.6, 124.8 (C-F, 1JC-F $=285.9 \mathrm{~Hz}), 121.8,81.2(\mathrm{C}-\mathrm{F}, 2 \mathrm{JC}-\mathrm{F}=28.3 \mathrm{~Hz})$. HRMS (ESI) $\mathrm{m} / \mathrm{z}:[\mathrm{M}-\mathrm{OH}]^{+}$calcd for $\mathrm{C}_{20} \mathrm{H}_{13} \mathrm{BrF}_{3} \mathrm{~S} 420.9868$; found 420.9872. FTIR (cm $\left.{ }^{-1}\right)$ 3651, 3033, 2365, 1569, 1471,C-F, 1JCF 1160, 1005, 910 . 


\section{(2-((4-Bromophenyl)thio)phenyl)(4-chlorophenyl)methanol (10a)}

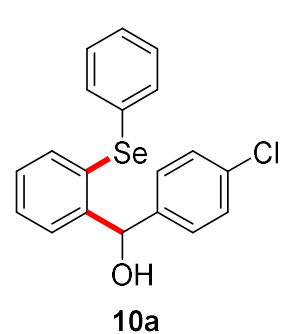

Following the general procedure, treatment of 4chlorobenzaldehyde 1a (0.035 g, $0.25 \mathrm{mmol})$, 2-(trimethylsilyl)phenyl trifluoromethanesulfonate $\mathbf{2 a}(0.149 \mathrm{~g}, 121 \mu \mathrm{L}, 0.5 \mathrm{mmol})$ and benzeneselenol 9a $(0.078 \mathrm{~g}, 0.5 \mathrm{mmol})$ in the presence of $\mathrm{KF}(0.073 \mathrm{~g}, 1.25$ mmol ) and 18-crown-6 $(0.330 \mathrm{~g}, 1.25 \mathrm{mmol})$ in THF $(1.0 \mathrm{~mL})$ at $25^{\circ} \mathrm{C}$ for

$12 \mathrm{~h}$ followed by purification via silica gel flash column chromatography (Pet. ether /EtOAc $=$ 90/10) of the crude reaction mixture afforded (2-((4-bromophenyl)selanyl)phenyl)(4chlorophenyl)methanol 10a as a light yellow sticky liquid (0.048 g, 51\% yield).

$\boldsymbol{R}_{\mathbf{f}}($ Pet. ether $/$ EtOAc $=90 / 10): 0.47 ;{ }^{1} \mathbf{H}$ NMR (400 MHz, $\left.\mathbf{C D C l}_{3}\right) \delta$ 7.48-7.42 (m, 2H), 7.36$7.31(\mathrm{~m}, 3 \mathrm{H}), 7.28-7.21(\mathrm{~m}, 7 \mathrm{H}), 7.20-7.16(\mathrm{~m}, 1 \mathrm{H}), 6.27$ (s, 1H), 2.58 (s, 1H). ${ }^{13}$ C NMR (100 MHz, CDCl3) $\delta 144.9,141.4,135.4,133.4,132.7,131.4,130.4,129.6,128.9,128.7,128.6$, 128.6, 127.9, 127.5, 74.6. HRMS (ESI) m/z: $\mathrm{M}^{+}$calcd for (mass of corresponding oxidized product using PDC) $\mathrm{C}_{19} \mathrm{H}_{13} \mathrm{ClOSe} 371.9820$; found 371.9808. FTIR (cm-1) 3384, 3056, 2923, $1575,1485,1435,1479,1091,1015$.

\section{Fe-Catalyzed Cyclisation of 4a}
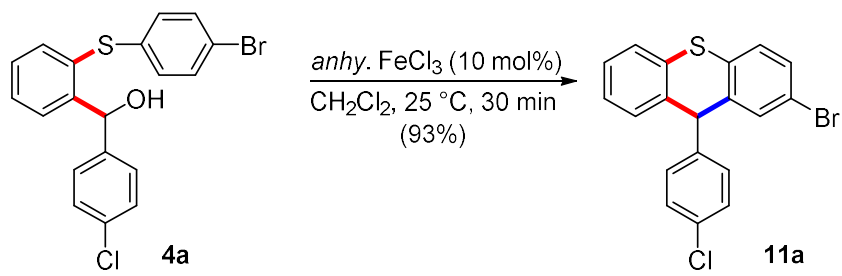

Following the literature procedure ${ }^{8}$ treatment of 2(2-((4-bromophenyl)thio)phenyl)(4chlorophenyl)methanol 4a $(0.101 \mathrm{~g}, 0.25 \mathrm{mmol})$ with $10 \mathrm{~mol} \% \mathrm{FeCl}_{3}(4 \mathrm{mg})$ in dry DCM (4 $\mathrm{mL}$ ) at $25^{\circ} \mathrm{C}$ for $30 \mathrm{~min}$ followed by purification via silica gel flash column chromatography (Pet. ether $/$ EtOAc $=95 / 5)$ of the crude reaction mixture afforded 2-bromo-9-(4-chlorophenyl)$9 H$-thioxanthene 11a as a white sticky liquid ( $0.09 \mathrm{~g}, 93 \%$ yield).

$\boldsymbol{R} \mathbf{f}\left(\right.$ Pet. ether $/$ EtOAc = 95/05): 0.41; ${ }^{1} \mathbf{H}$ NMR (400 MHz, CDCl3) $\delta 7.52$ (s, 1H), 7.43-7.22 (m, 6H), $7.14(\mathrm{~d}, J=8.4 \mathrm{~Hz}, 2 \mathrm{H}), 6.90(\mathrm{~d}, J=8.3 \mathrm{~Hz}, 2 \mathrm{H}), 5.21$ (s, 1H). ${ }^{13} \mathbf{C}$ NMR (100 MHz, $\left.\mathbf{C D C l}_{3}\right) \delta 139.1,138.8,136.4,132.8,132.8,132.6,132.3,130.2,129.7,129.2,128.8,128.6$, 127.4, 127.1, 120.4, 77.5, 77.2, 76.8, 52.3. HRMS (ESI) m/z: [M] calcd for $\mathrm{C}_{19} \mathrm{H}_{12} \mathrm{BrClS}$ 385.9532; found 385.9514. FTIR ( $\left.\mathbf{c m}^{-1}\right)$ 2364, 1748, 1578, 1489, 1462, 1395, 1089, 1011, 900.

\footnotetext{
${ }^{8}$ Das, S. K.; Singh, R.; Panda, G. Eur. J. Org. Chem. 2009, 4757.
} 
10. ${ }^{1} \mathrm{H}$ and ${ }^{13} \mathrm{C}$ NMR Spectra of Products

(2-((4-Bromophenyl)thio)phenyl)(4-chlorophenyl)methanol (4a)
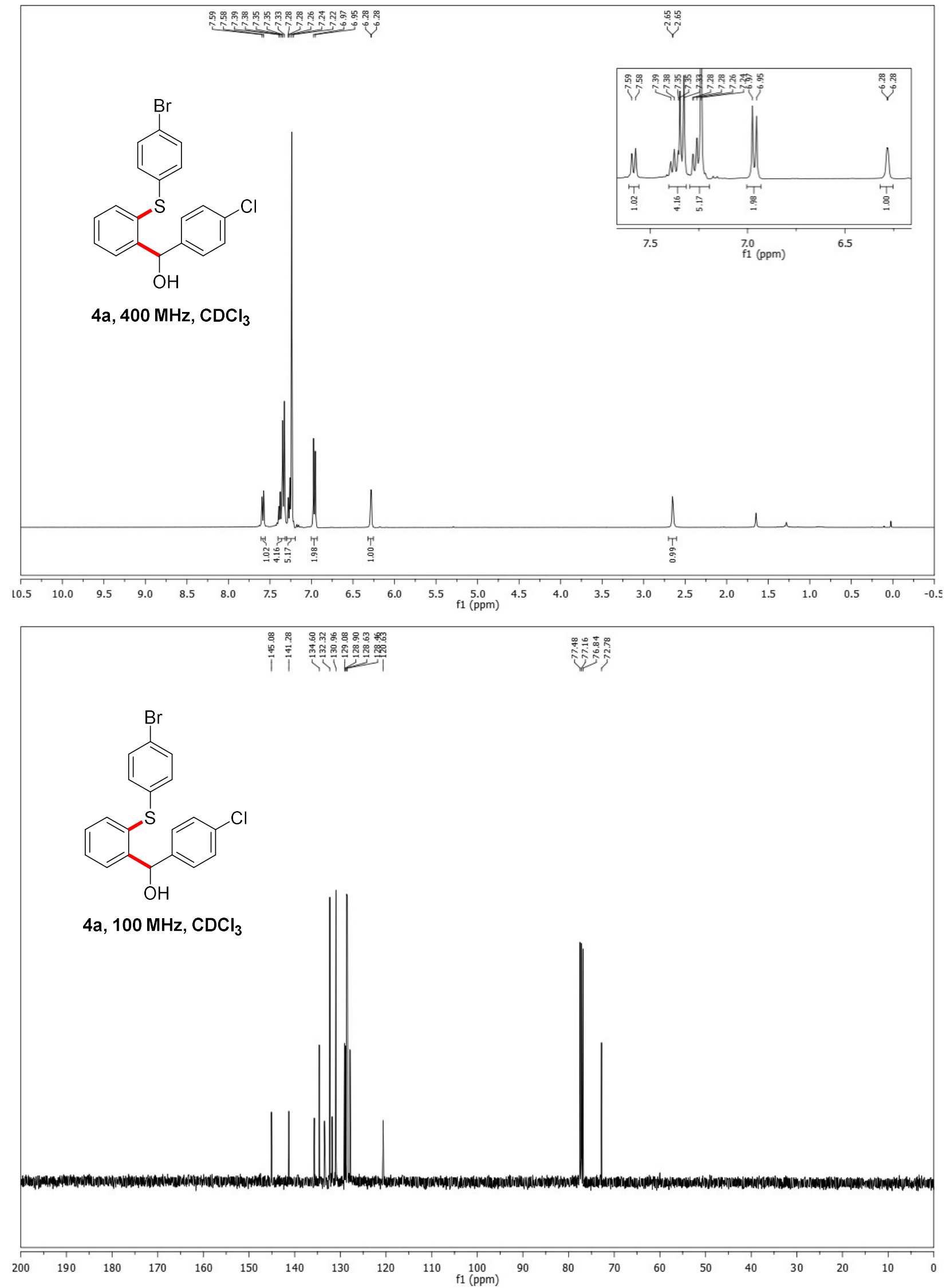


\section{2-((4-Bromophenyl)thio)phenyl)(4-methoxyphenyl)methanol (4b)}
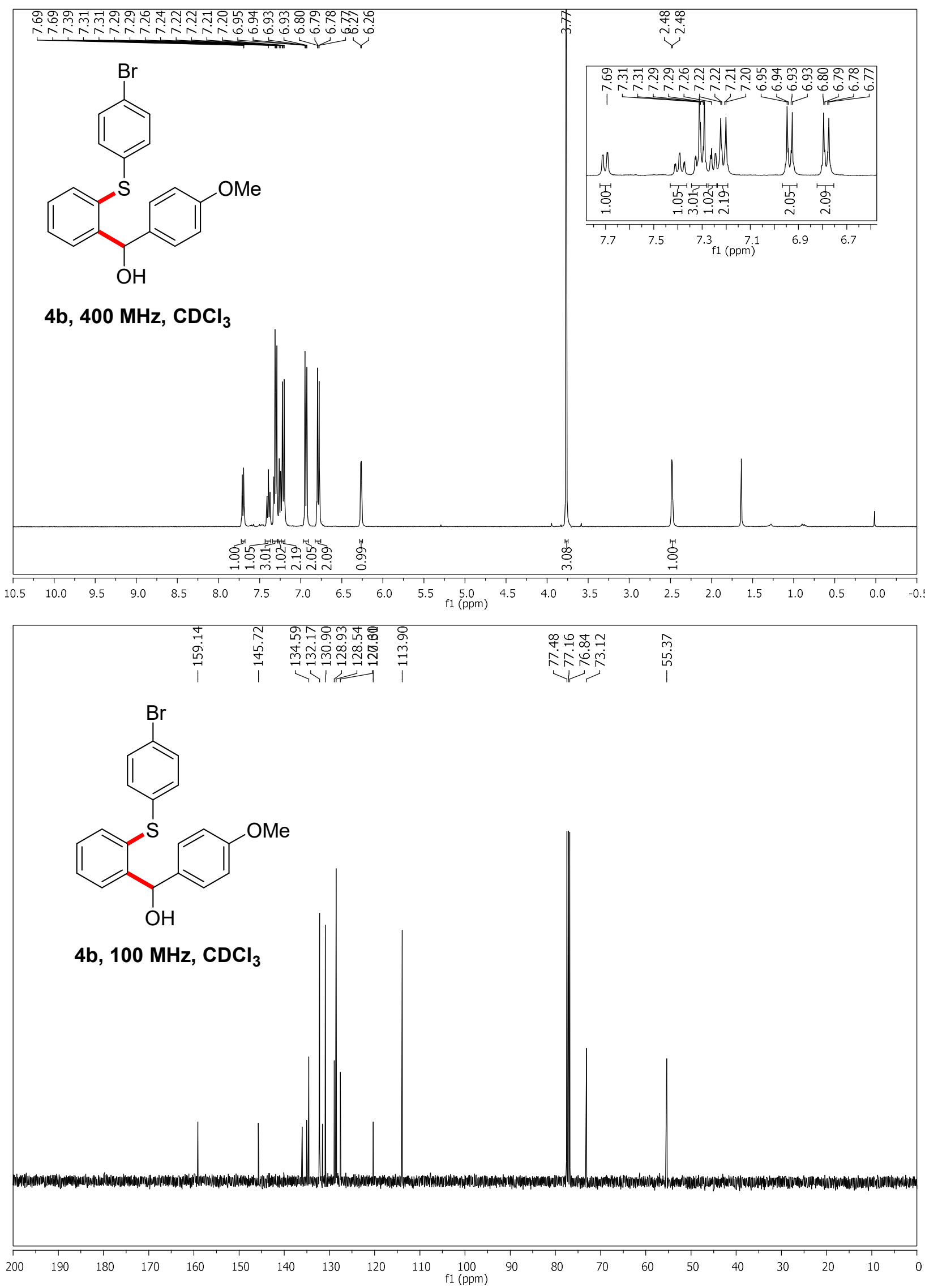
(2-((4-Bromophenyl)thio)phenyl)(p-tolyl)methanol (4c)
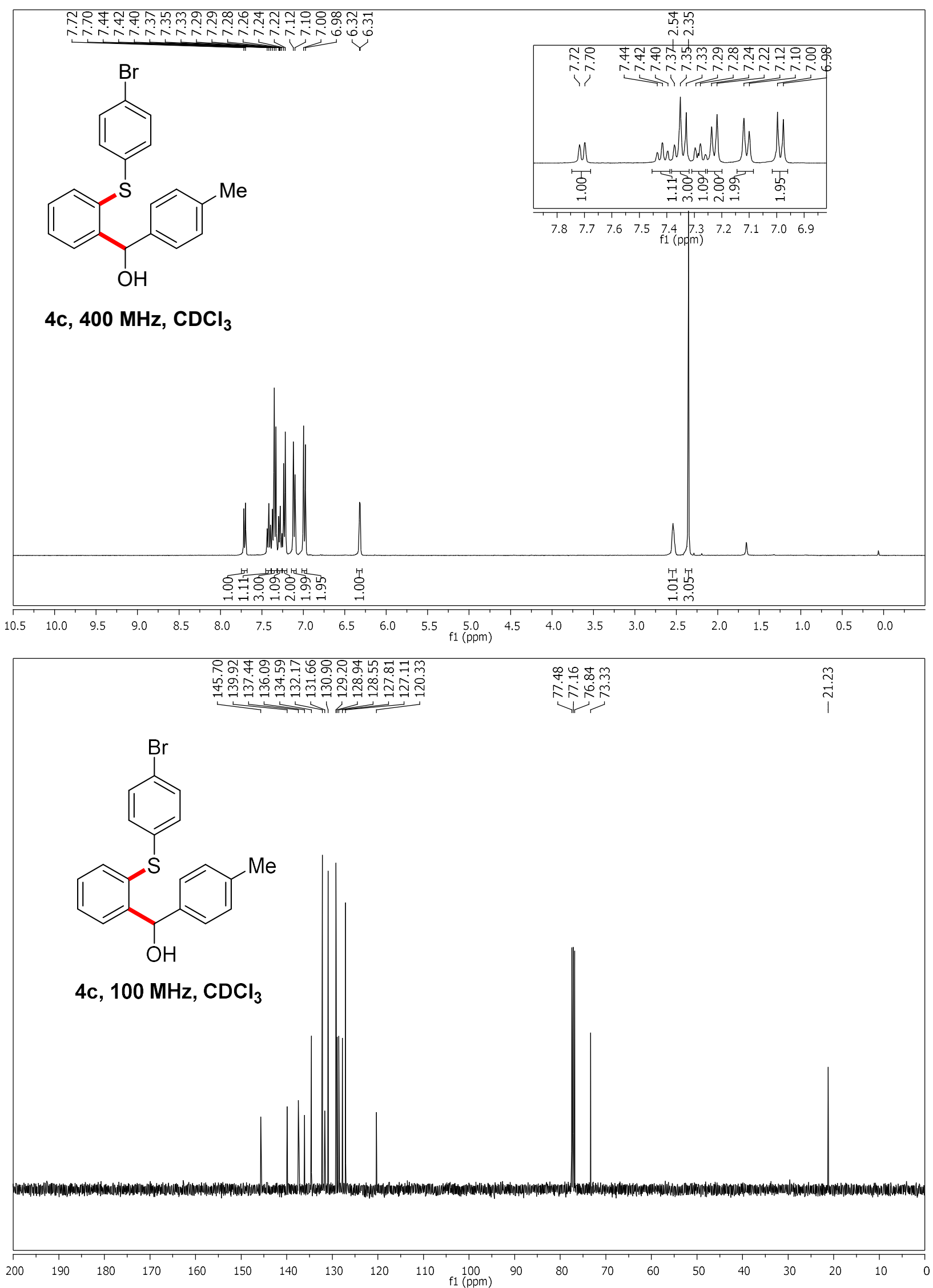
(2-((4-Bromophenyl)thio)phenyl)(phenyl)methanol (4d)
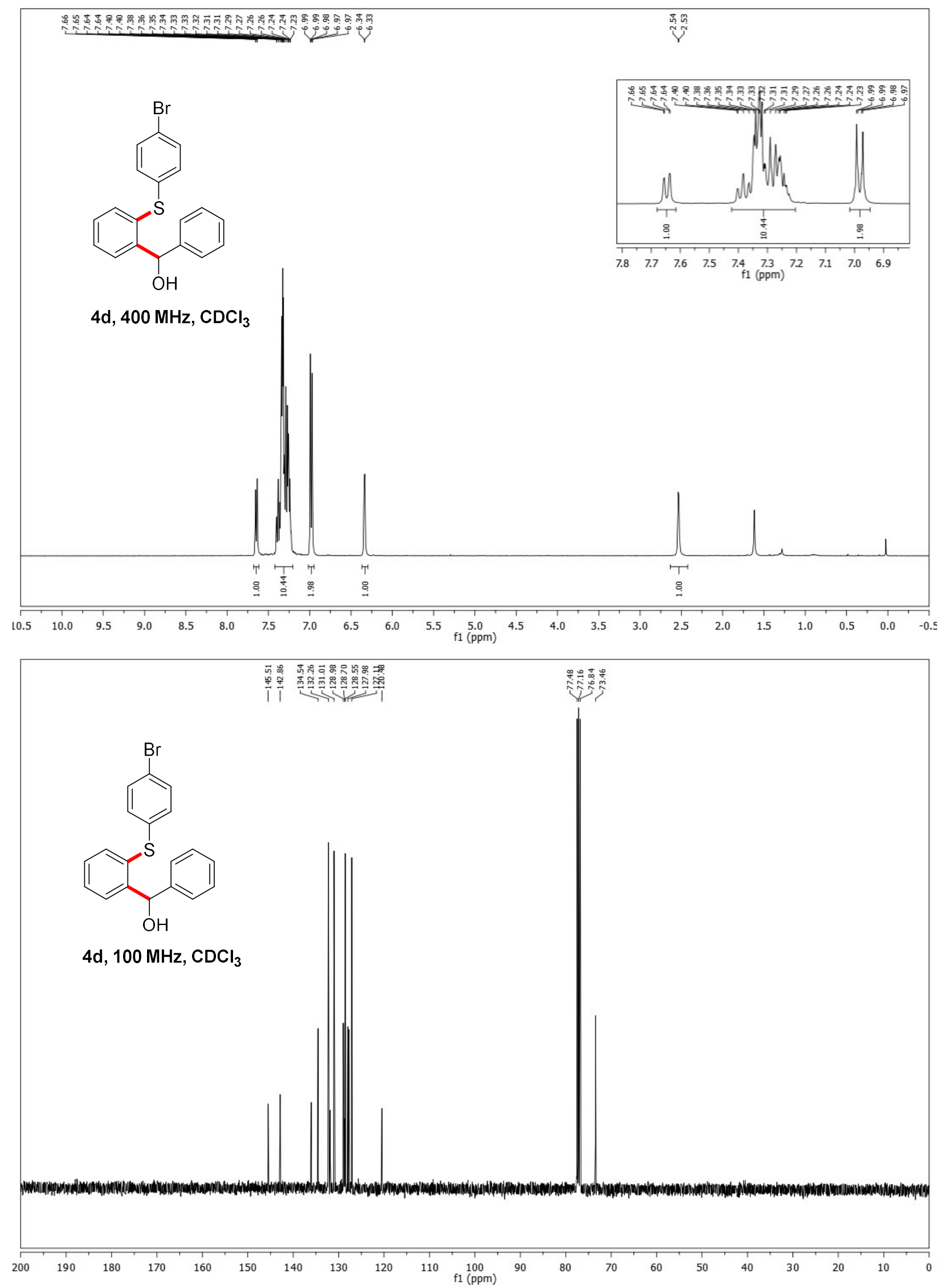
4-((2-((4-Bromophenyl)thio)phenyl)(hydroxy)methyl)benzonitrile (4e)
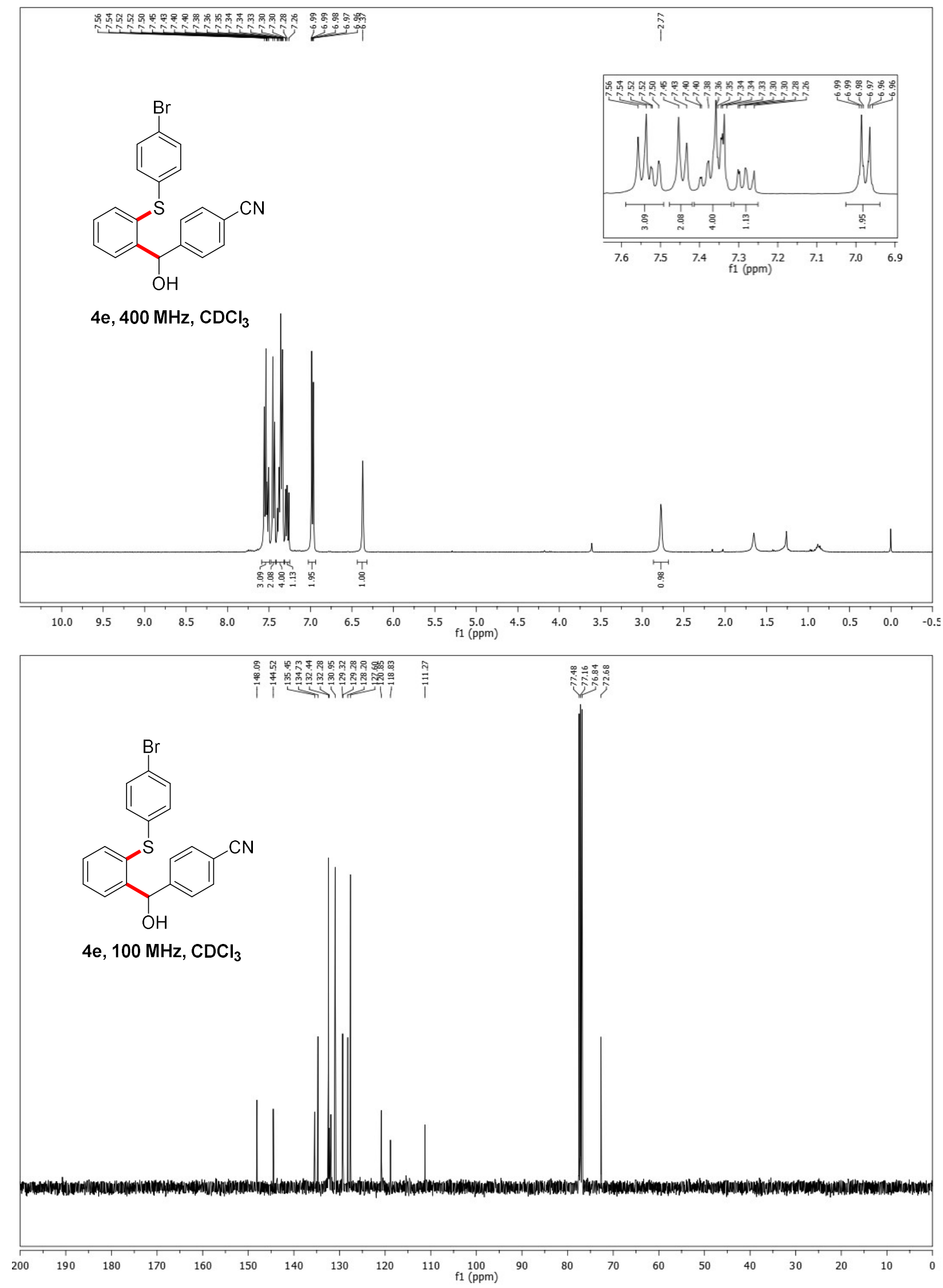
Methyl-4-((2-((4-bromophenyl)thio)phenyl)(hydroxy)methyl)benzoate (4f)
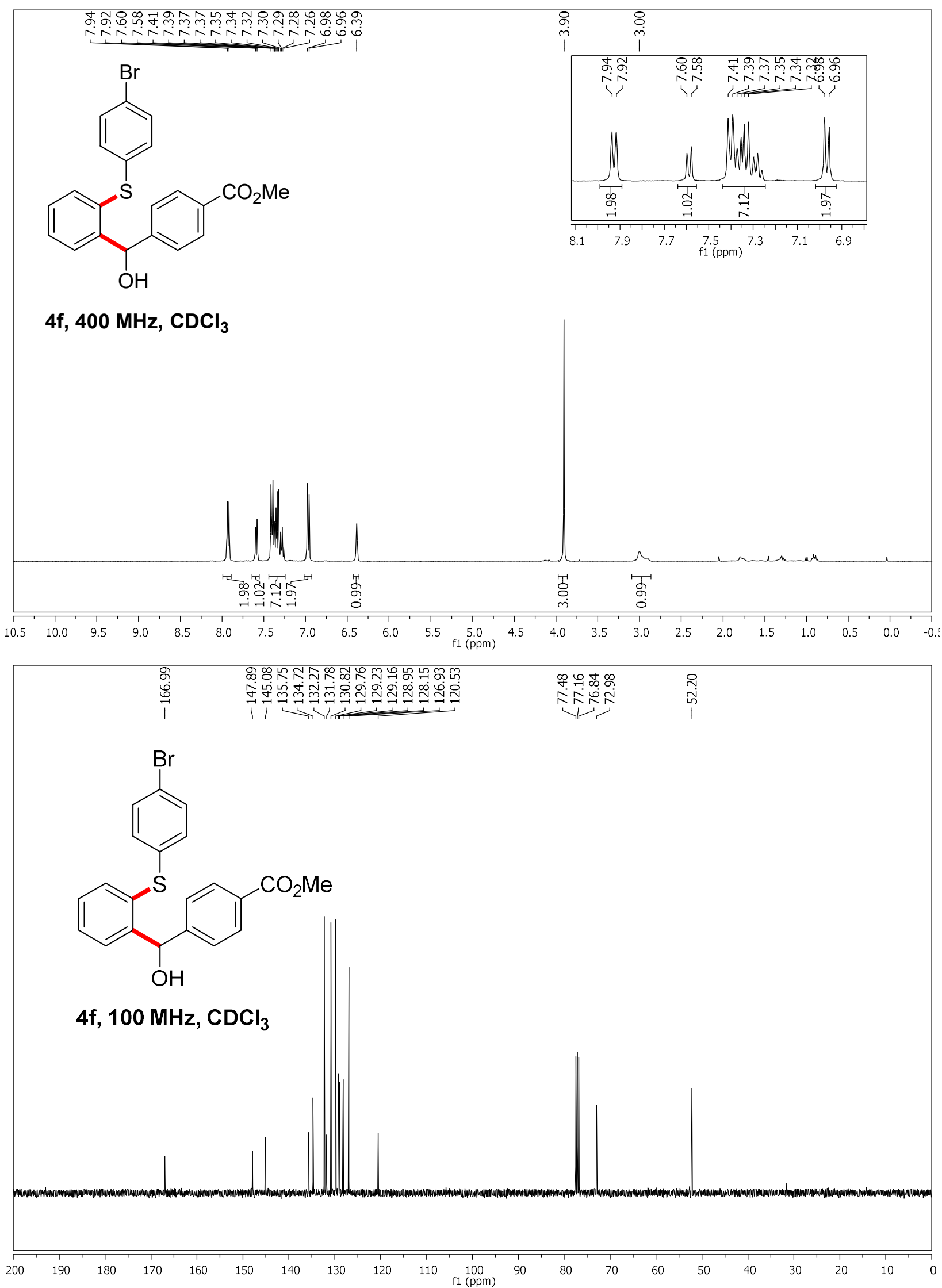
4-((2-((4-Bromophenyl)thio)phenyl)(hydroxy)methyl)benzaldehyde (4g)
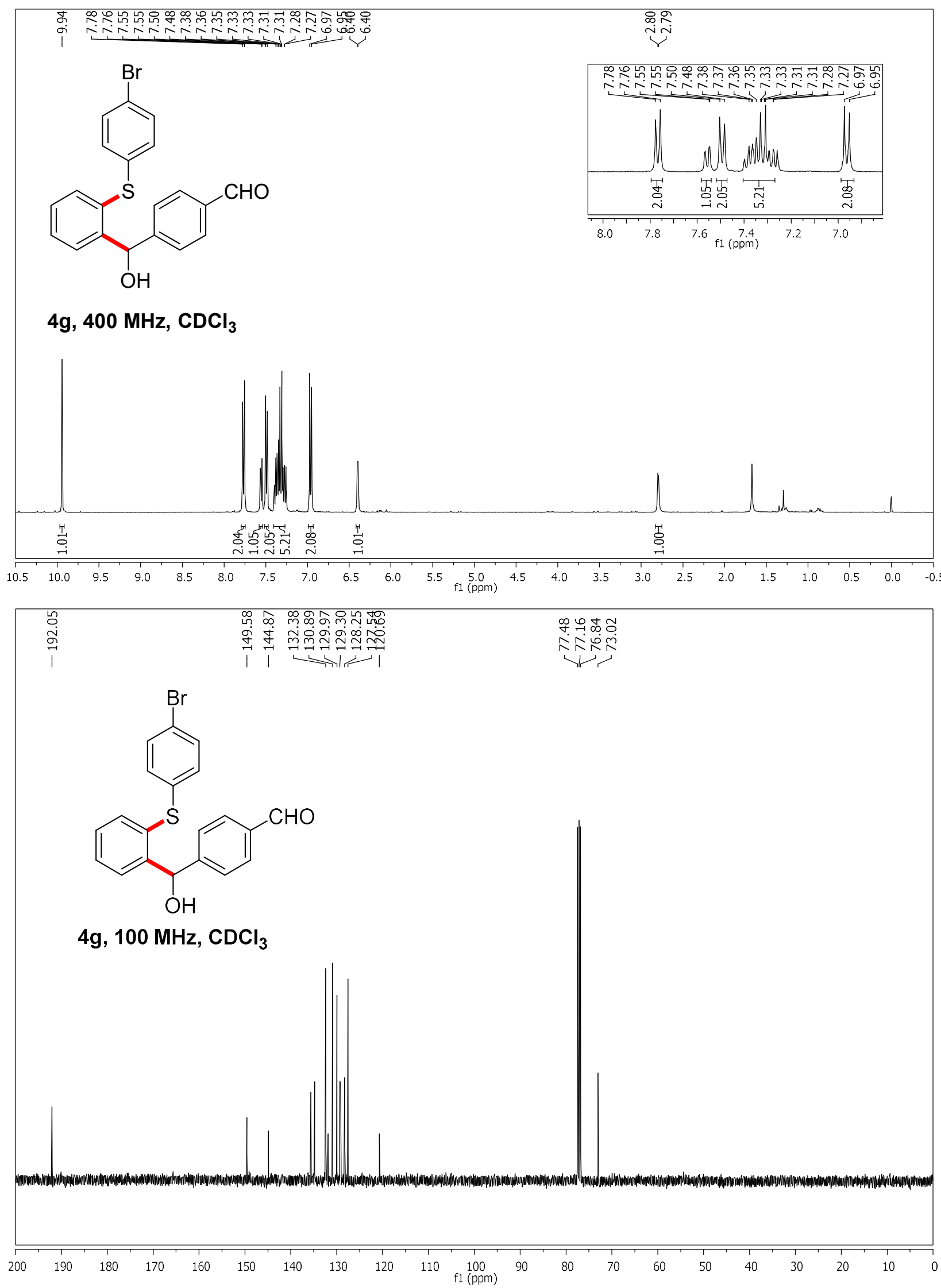
(2-((4-Bromophenyl)thio)phenyl)(m-tolyl)methanol (4h)
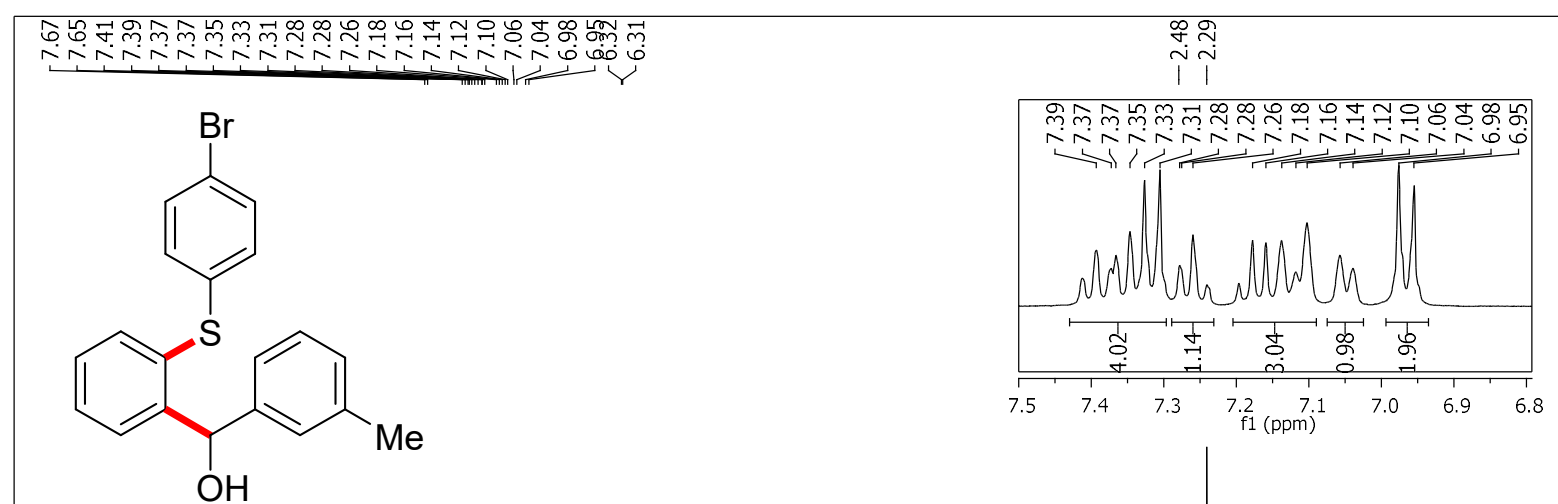

4h, $400 \mathrm{MHz}, \mathrm{CDCl}_{3}$
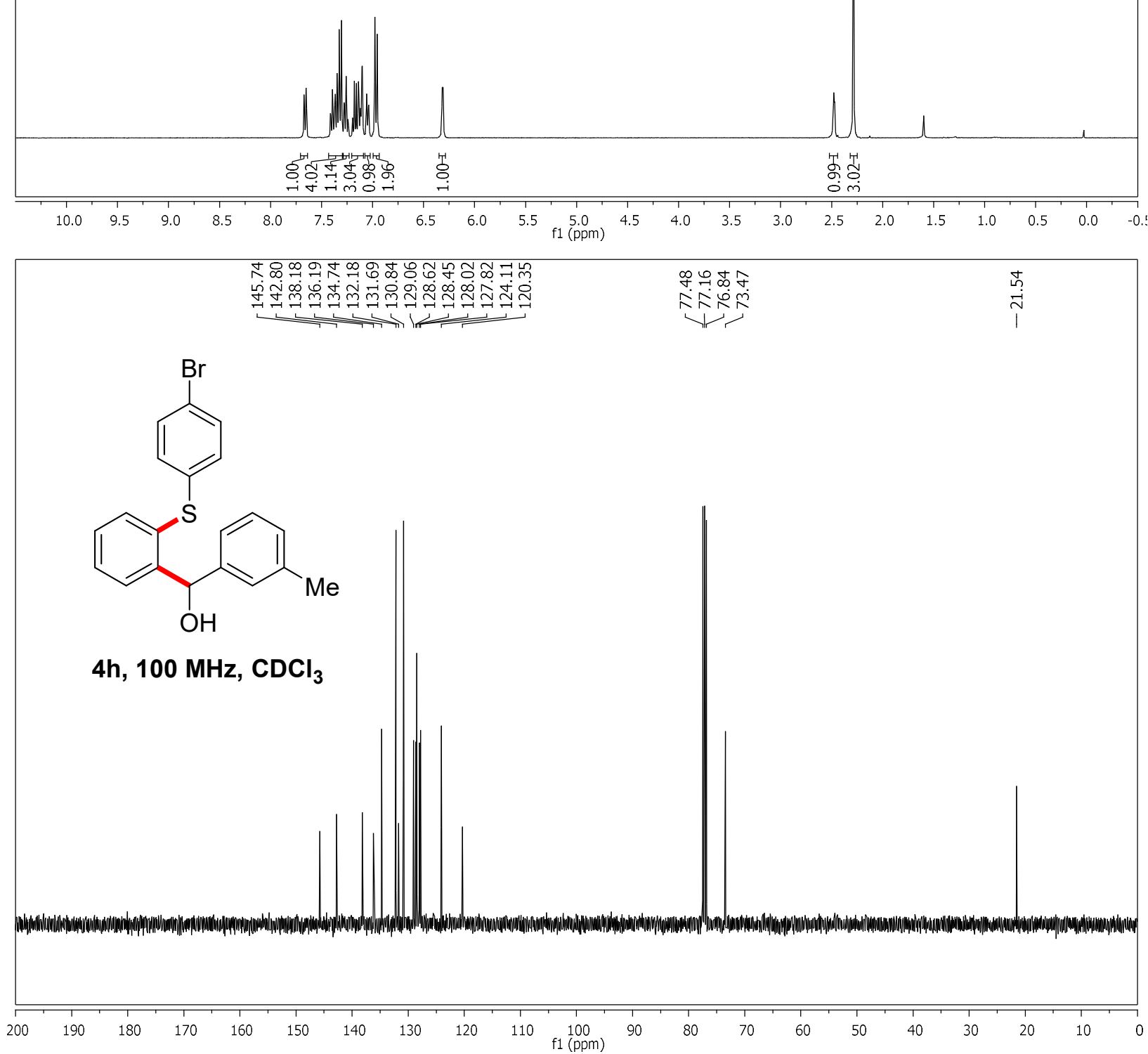
(3-Bromophenyl)(2-((4-bromophenyl)thio)phenyl)methanol (4i)
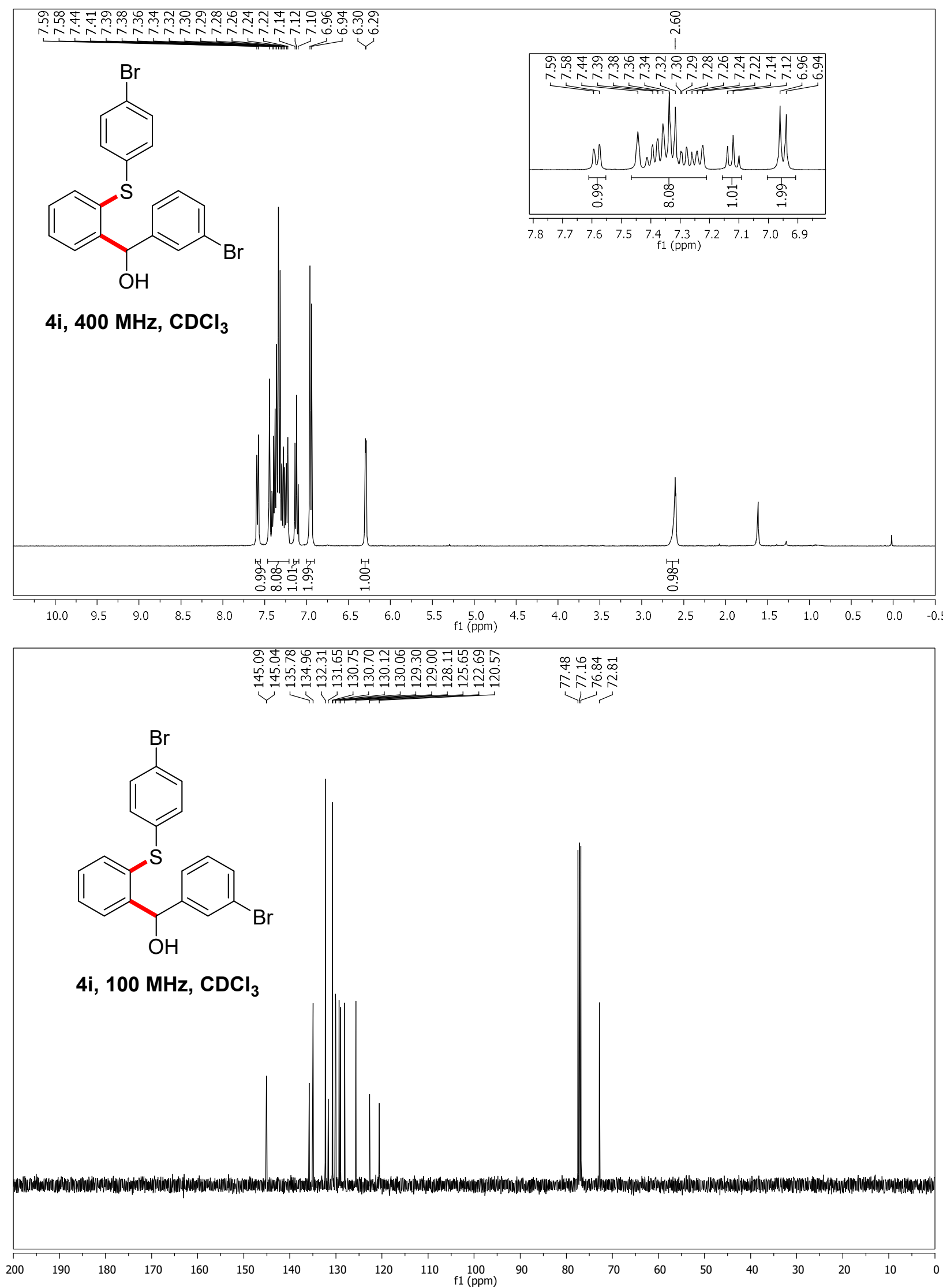
(2-((4-Bromophenyl)thio)phenyl)(3-chlorophenyl)methanol (4j)
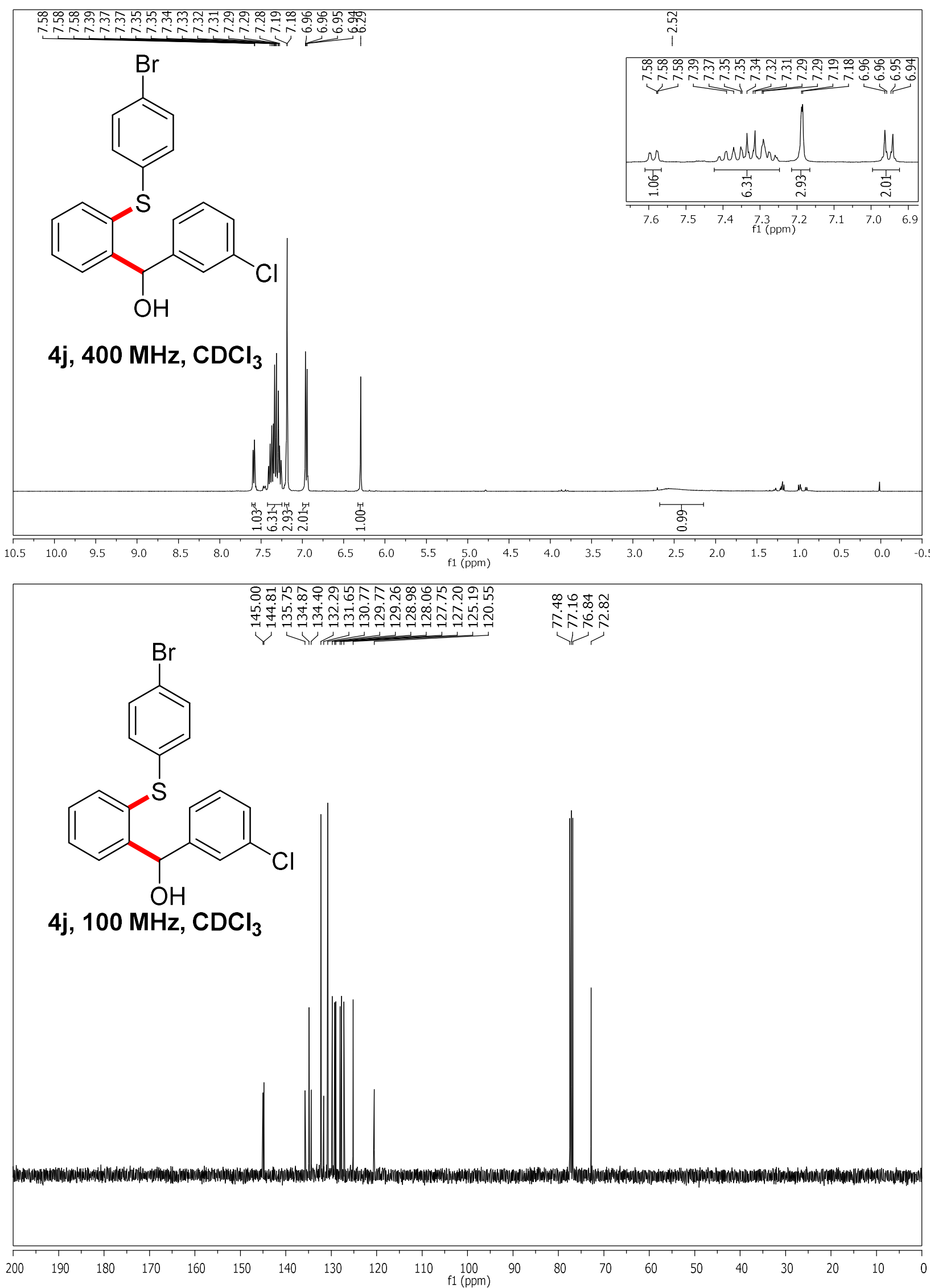
(2-((4-Bromophenyl)thio)phenyl)(3-nitrophenyl)methanol (4k)
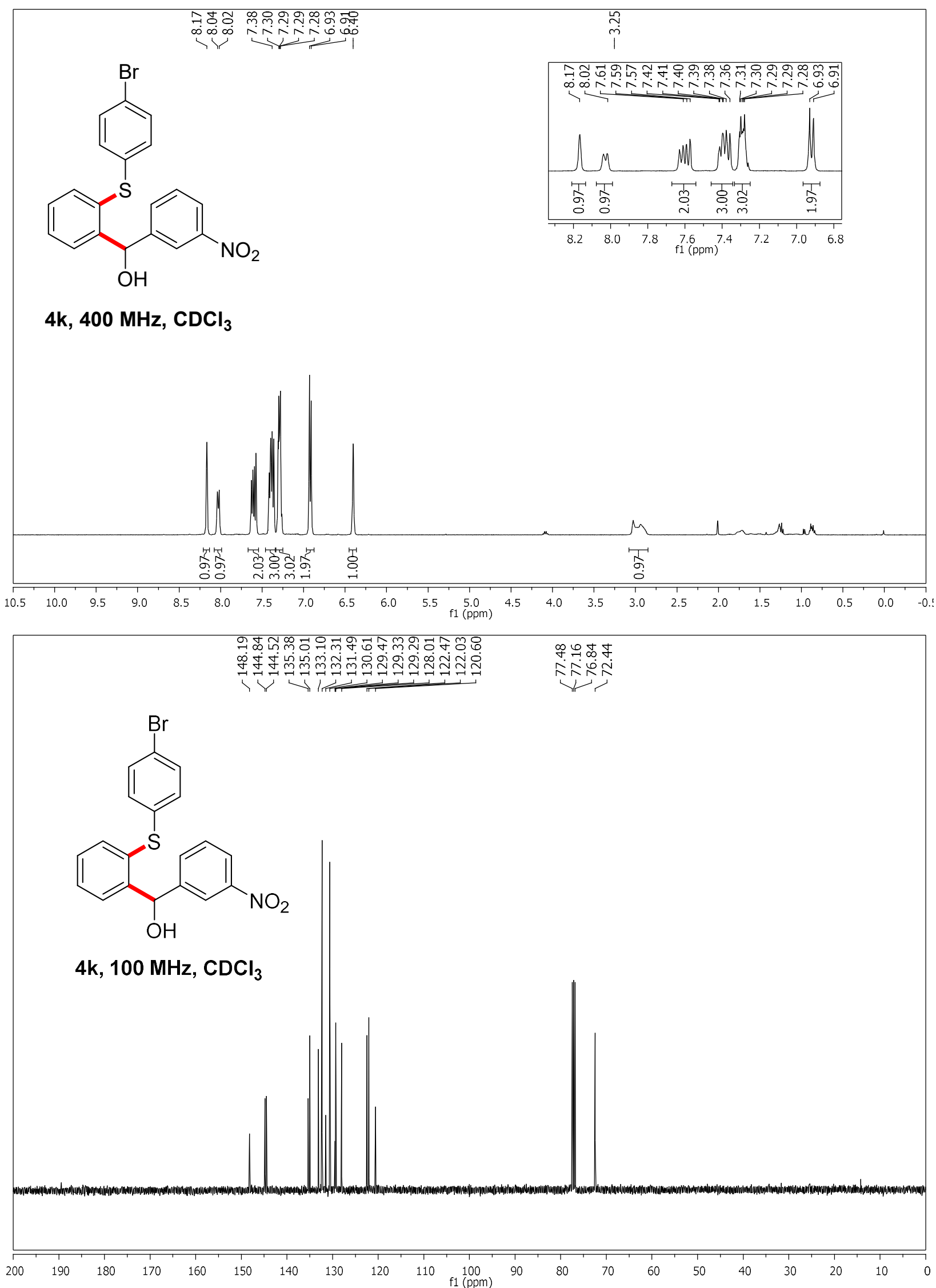
(2-((4-Bromophenyl)thio)phenyl)(o-tolyl)methanol (4I)
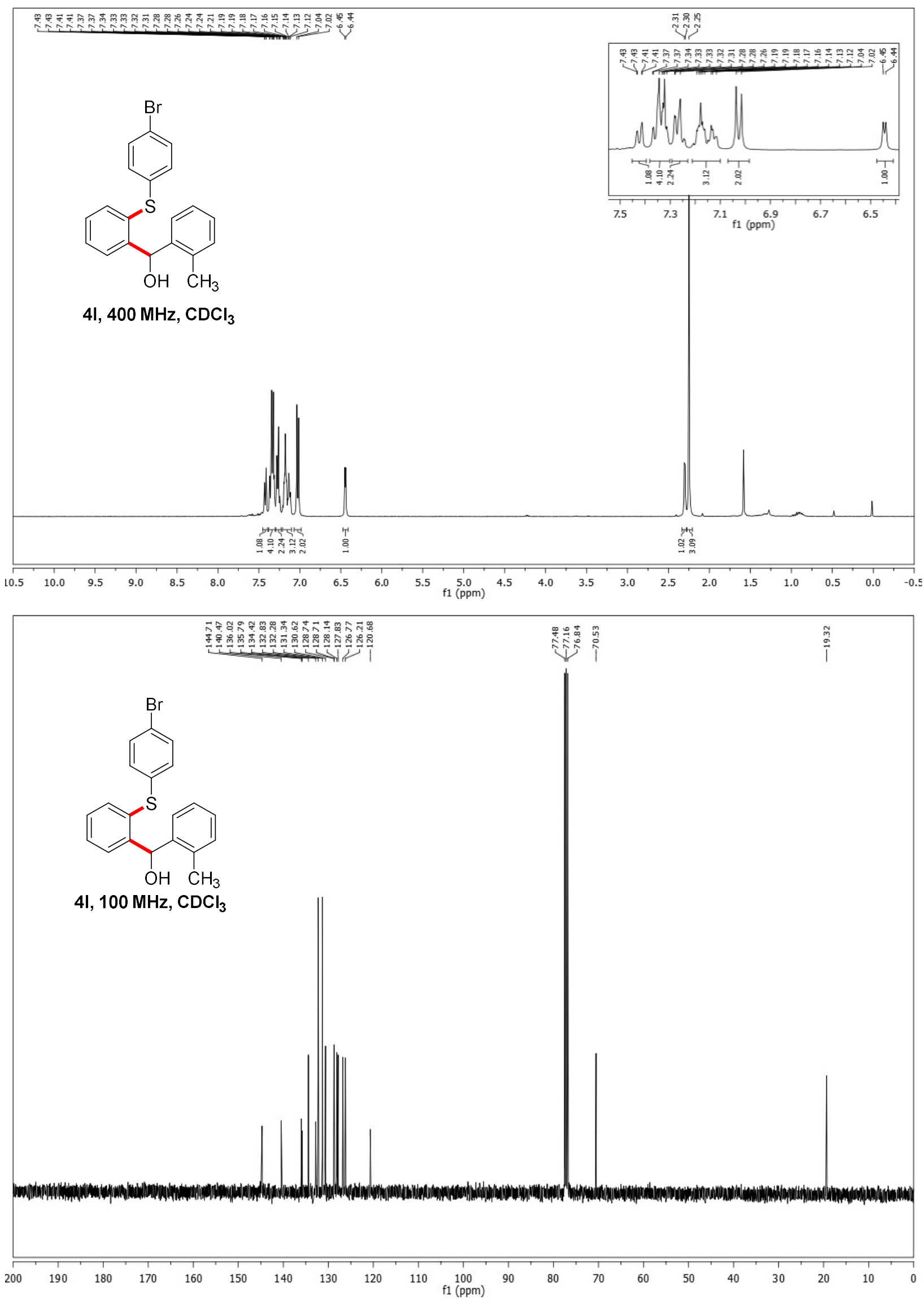
(2-((4-Bromophenyl)thio)phenyl)(2-chlorophenyl)methanol (4m)
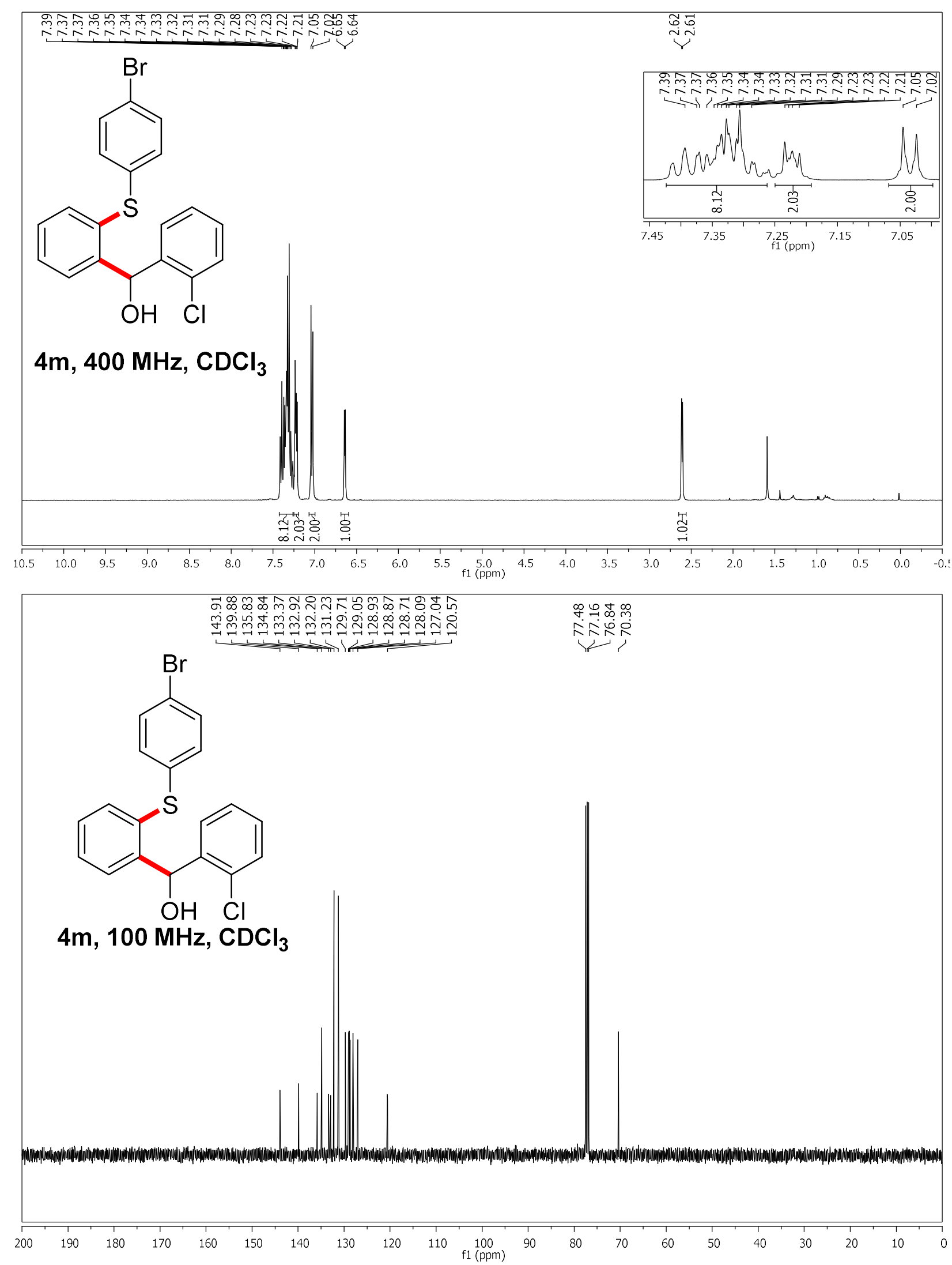
(2-((4-Bromophenyl)thio)phenyl)(naphthalen-2-yl)methanol (4n)
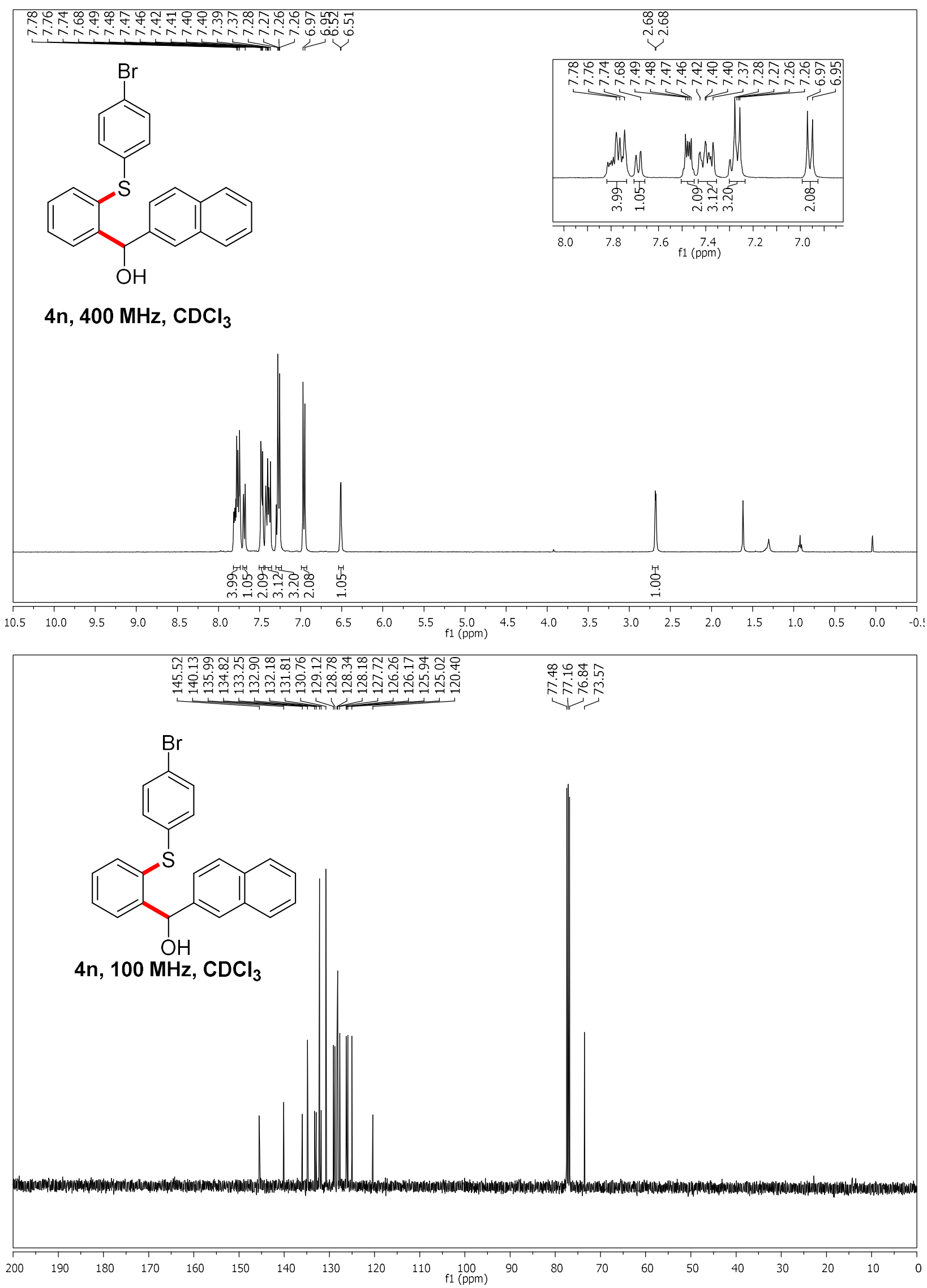
(2-((4-Bromophenyl)thio)phenyl)(3,5-dibromophenyl)methanol (40)
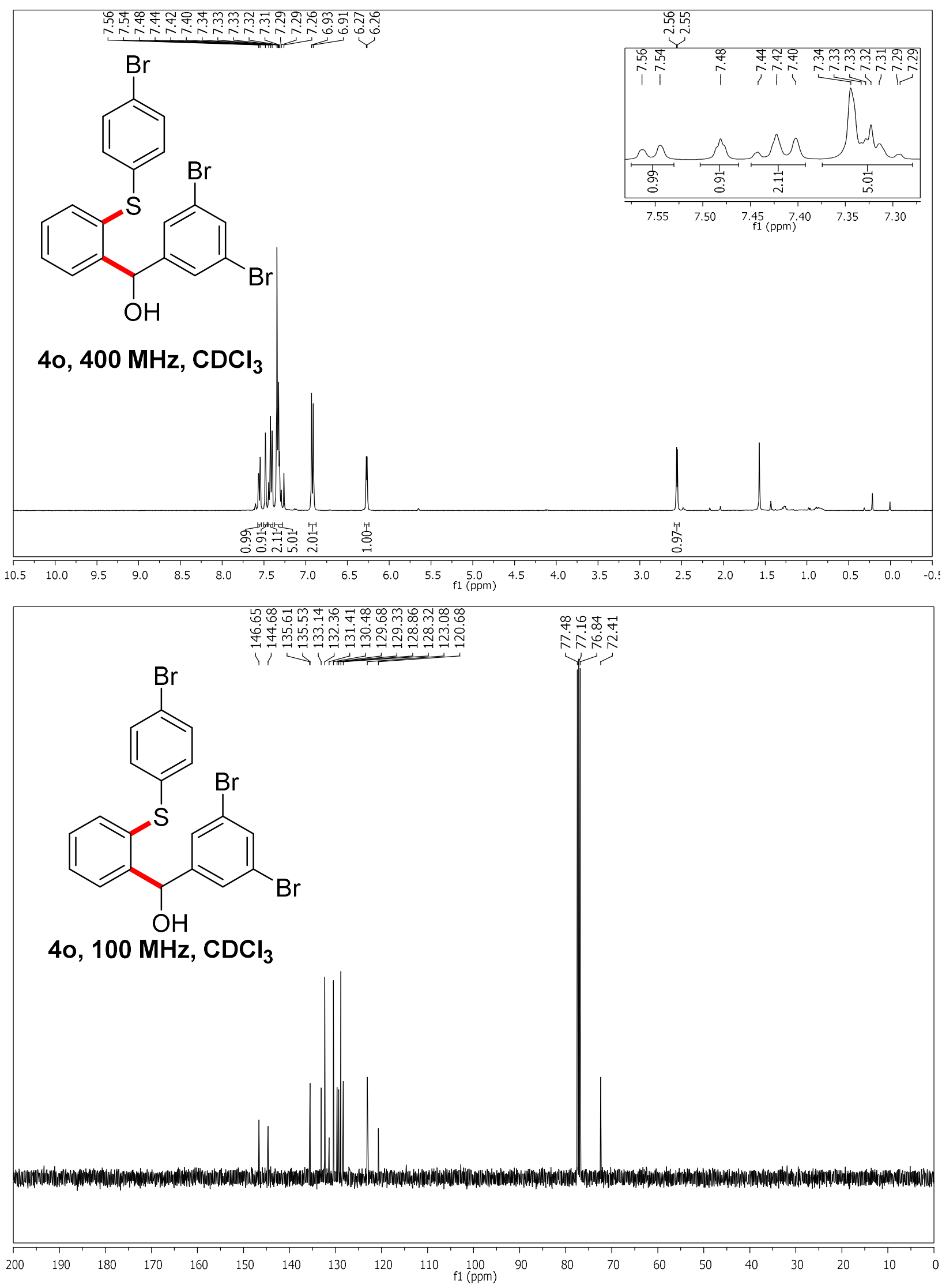


\section{(2-((4-Bromophenyl)thio)phenyl)(pyren-1-yl)methanol (4p)}
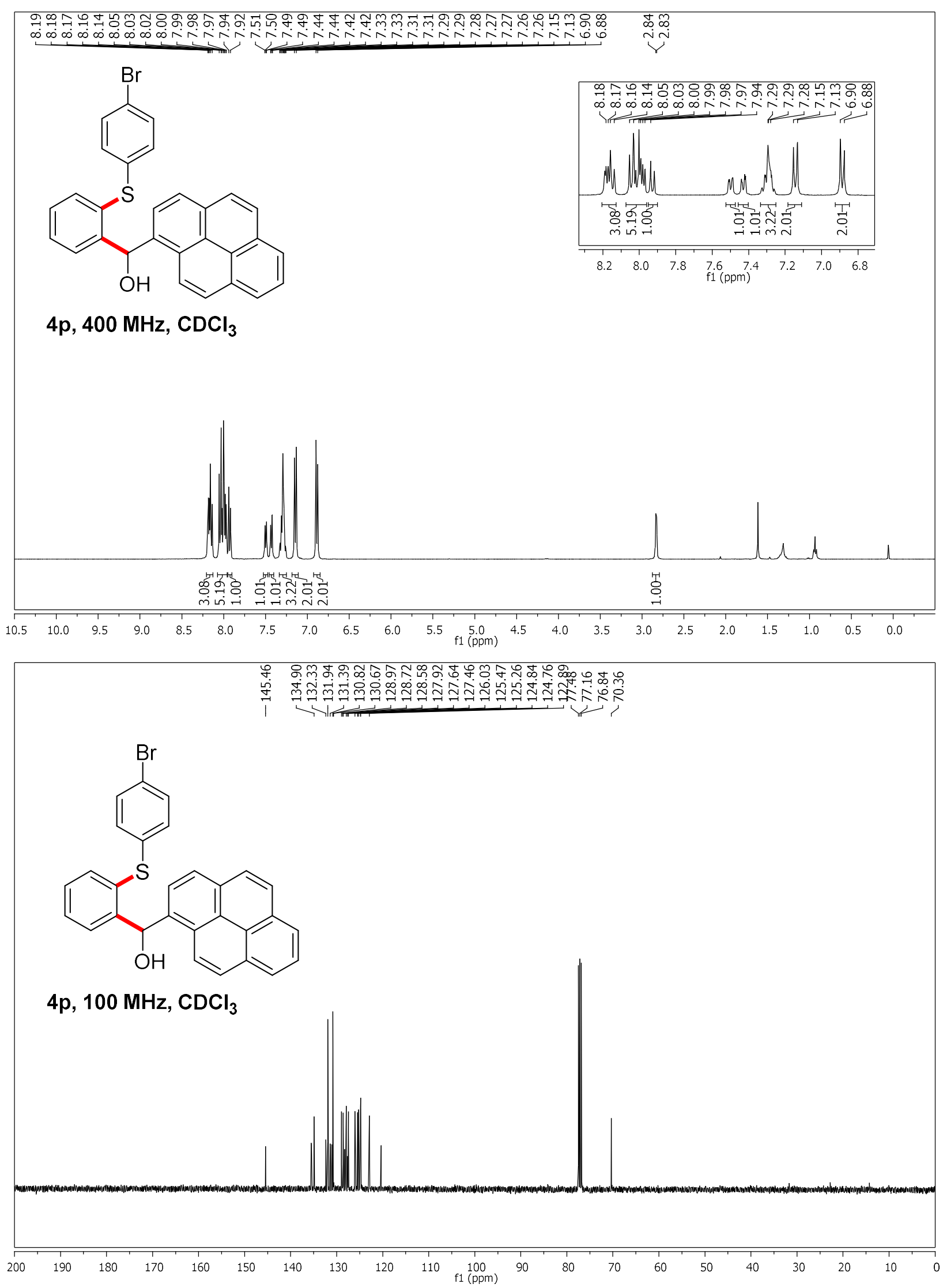
(2-((4-Bromophenyl)thio)phenyl)(thiophen-3-yl)methanol (4q)
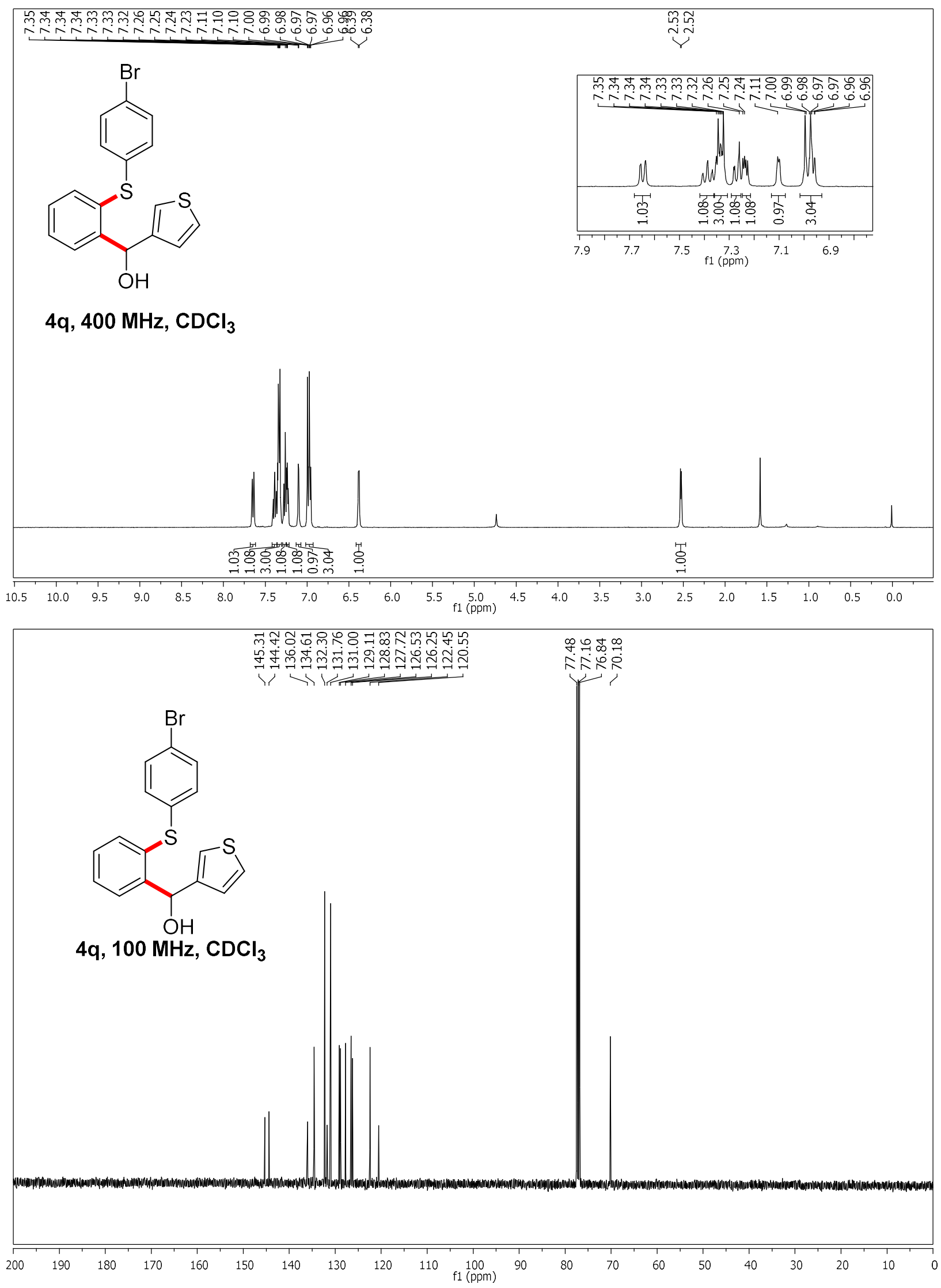
(2-((4-Bromophenyl)thio)phenyl)(thiophen-2-yl)methanol (4r)
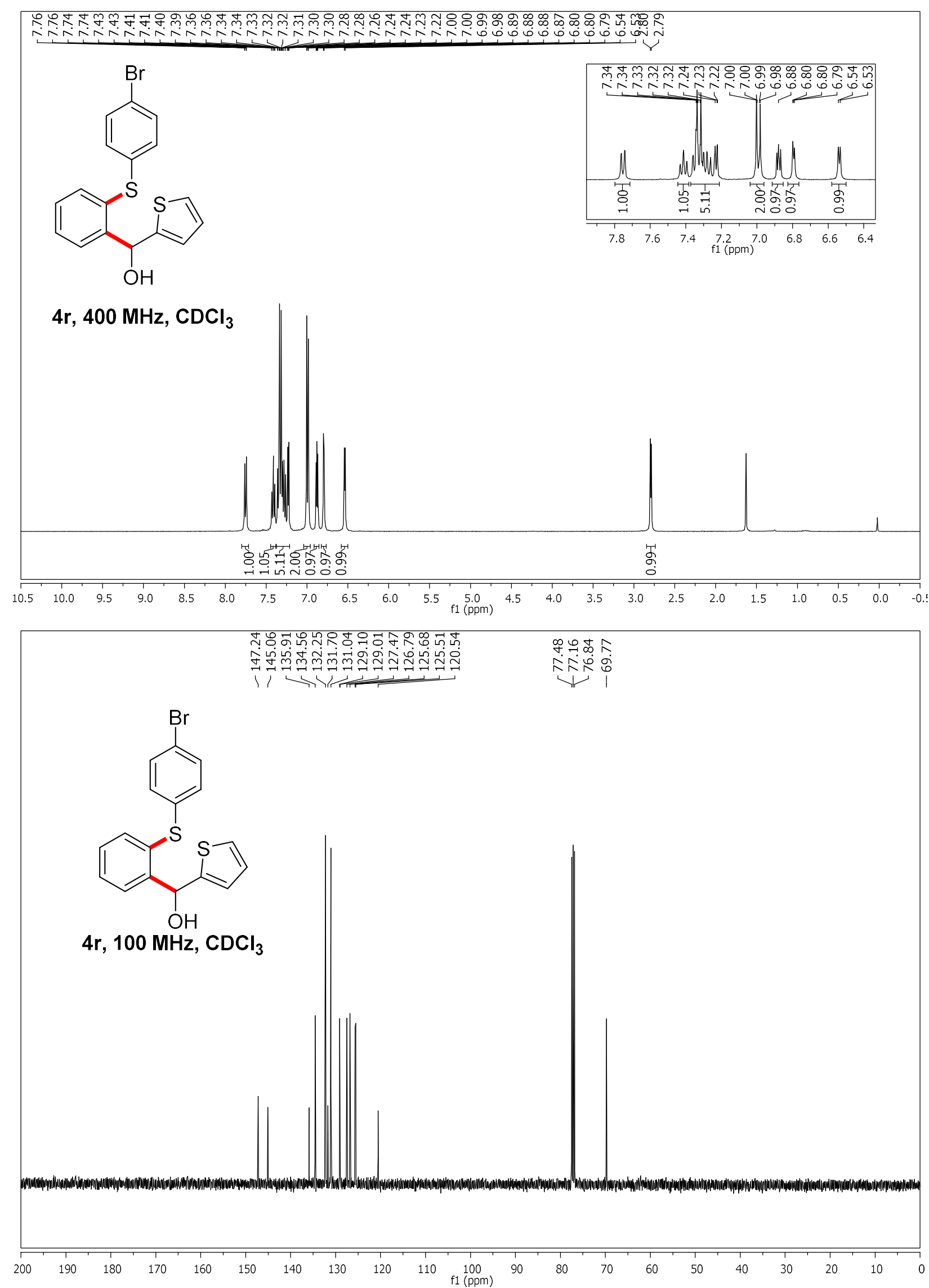
Benzofuran-2-yl(2-((4-bromophenyl)thio)phenyl)methanol (4s)
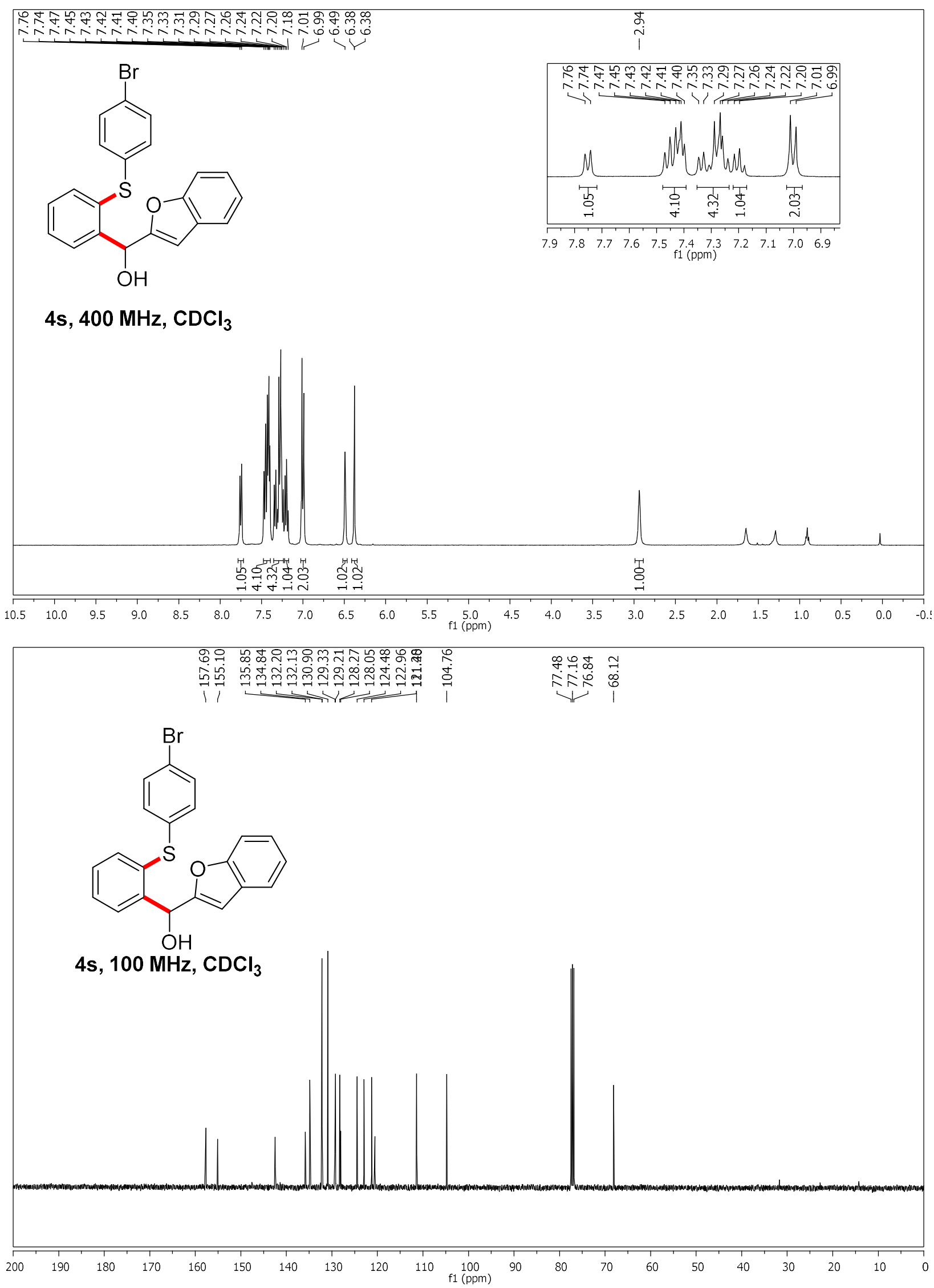
Benzo $[b]$ thiophen-3-yl(2-((4-bromophenyl)thio)phenyl)methanol (4t)
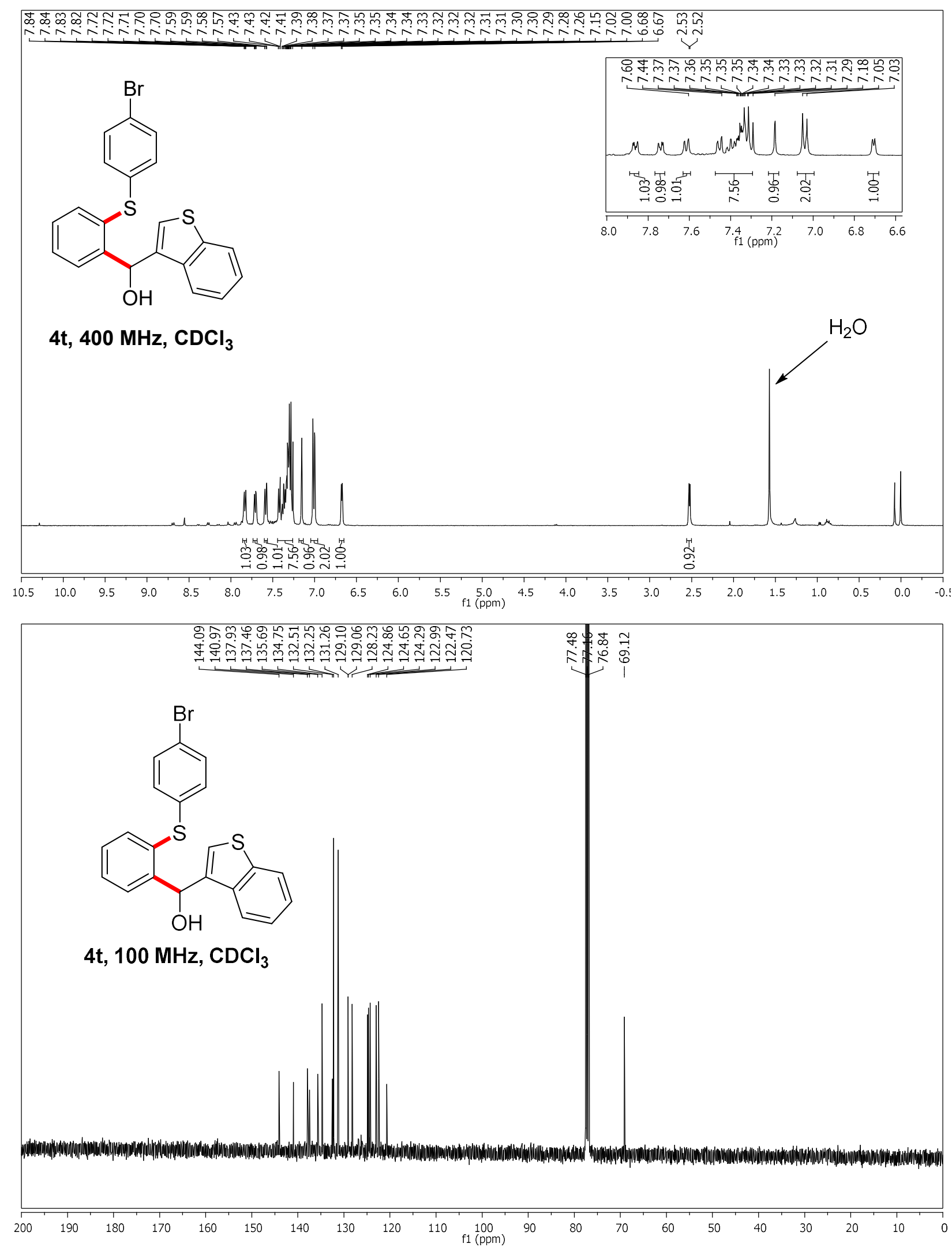
(E)-1-(2-((4-Bromophenyl)thio)phenyl)-3-phenylprop-2-en-1-ol (4u)
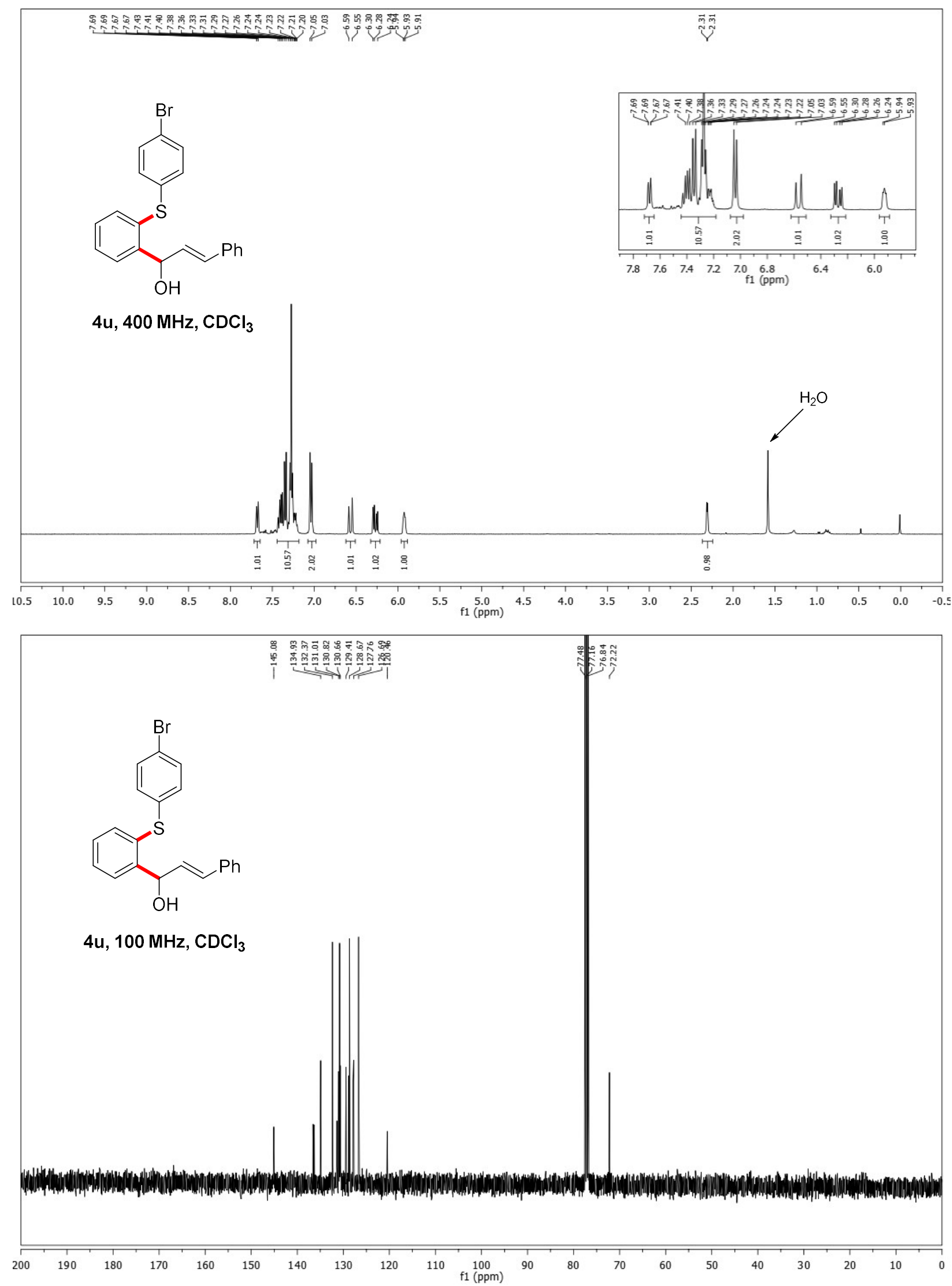
1-(2-((4-Bromophenyl)thio)phenyl)-3-phenylpropan-1-ol (4v)
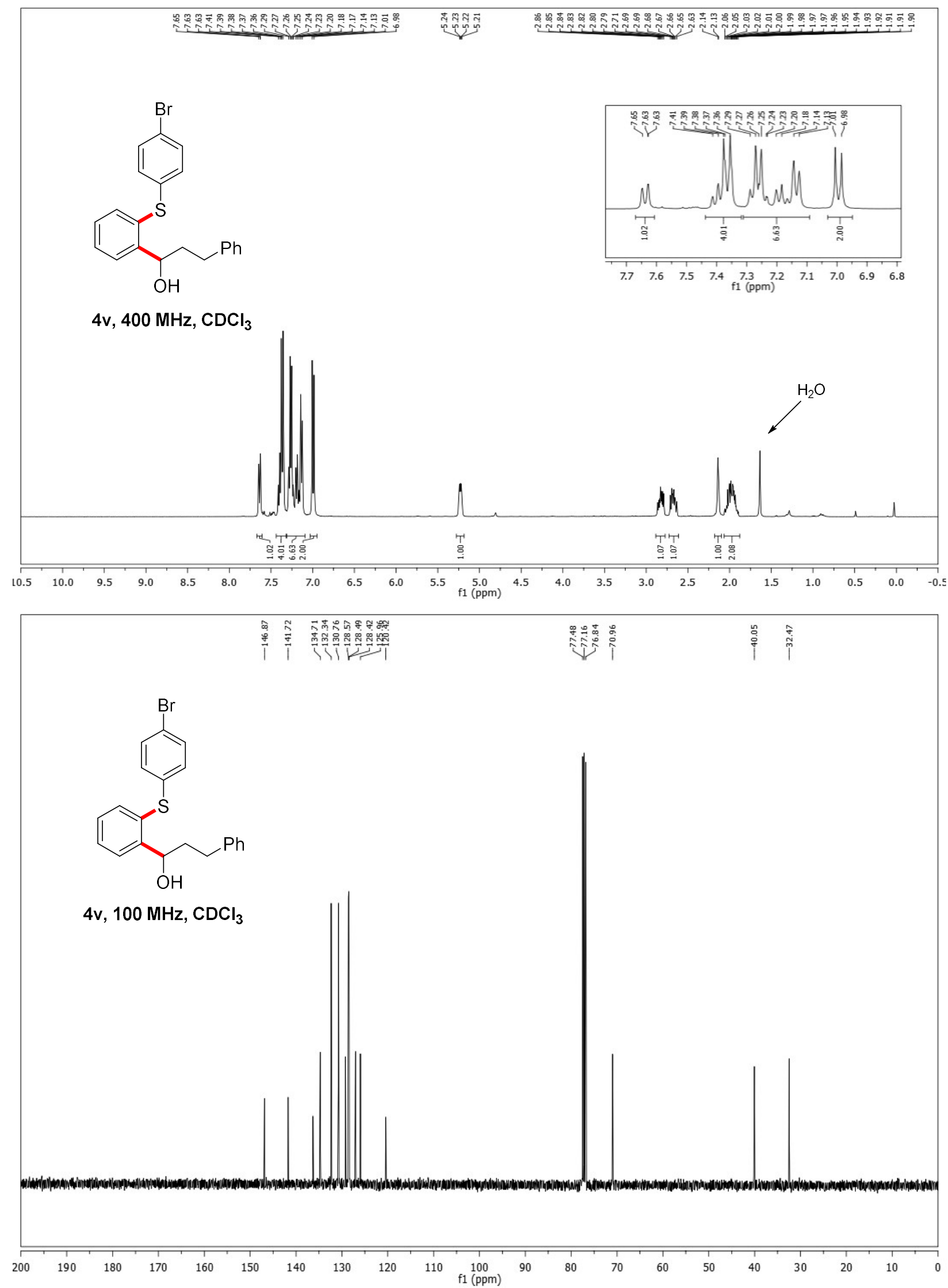
(2-((4-Bromophenyl)thio)phenyl)(cyclohexyl)methanol (4w)
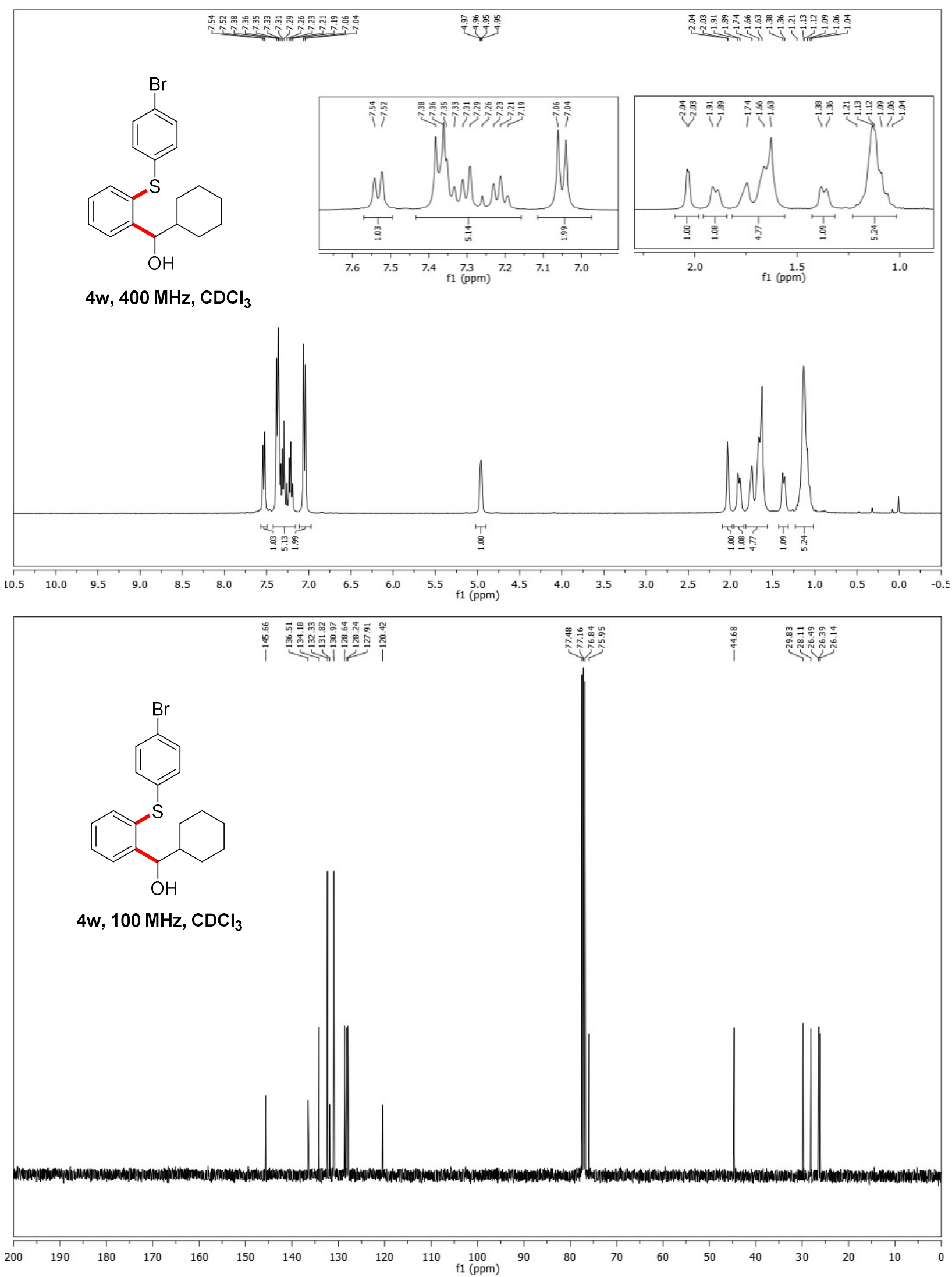
1-(2-((4-Bromophenyl)thio)phenyl)-2-methylpropan-1-ol (4x)
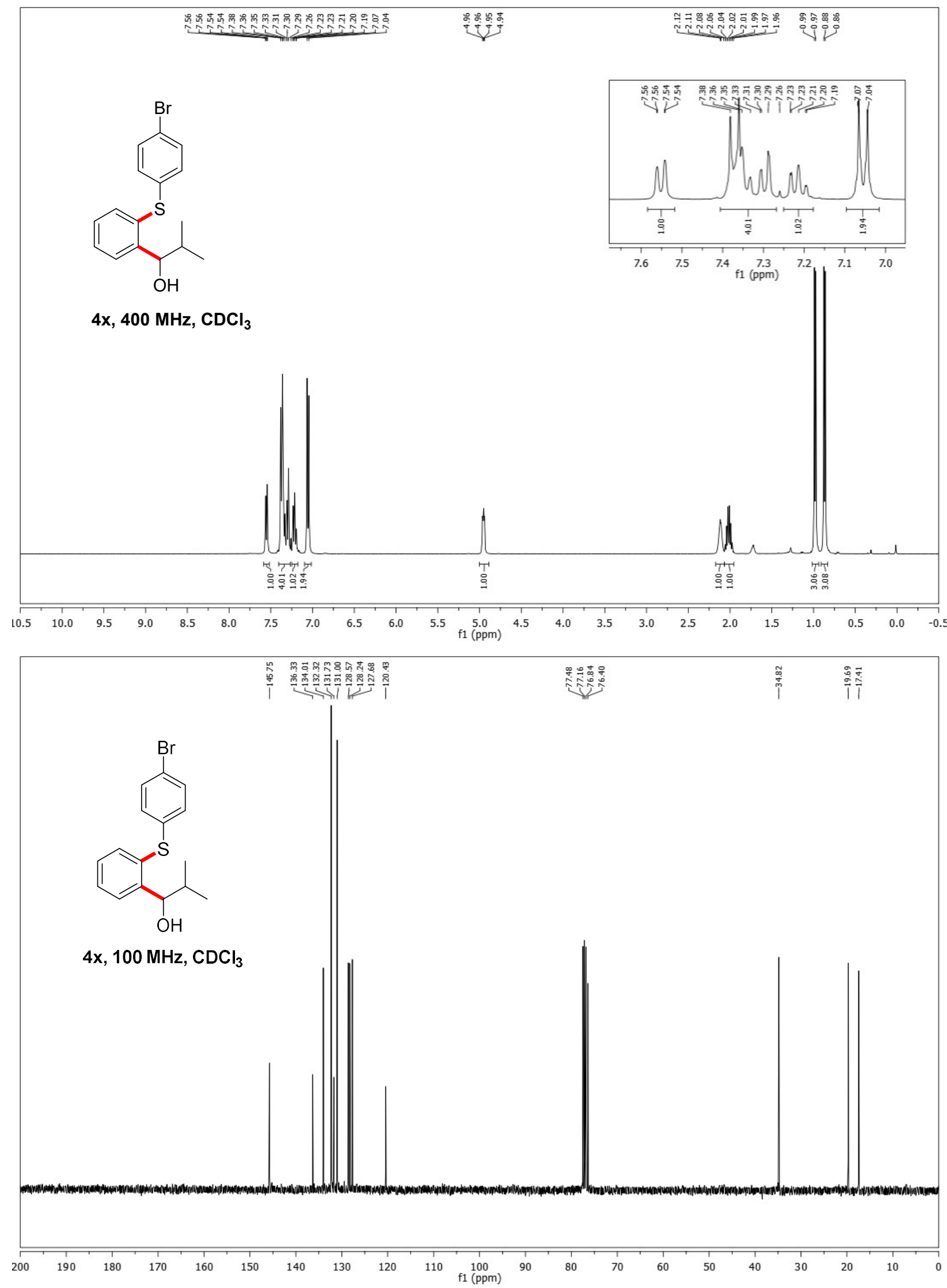


\section{1,4-Phenylenebis((2-((4-bromophenyl)thio)phenyl)methanol) (4y)}
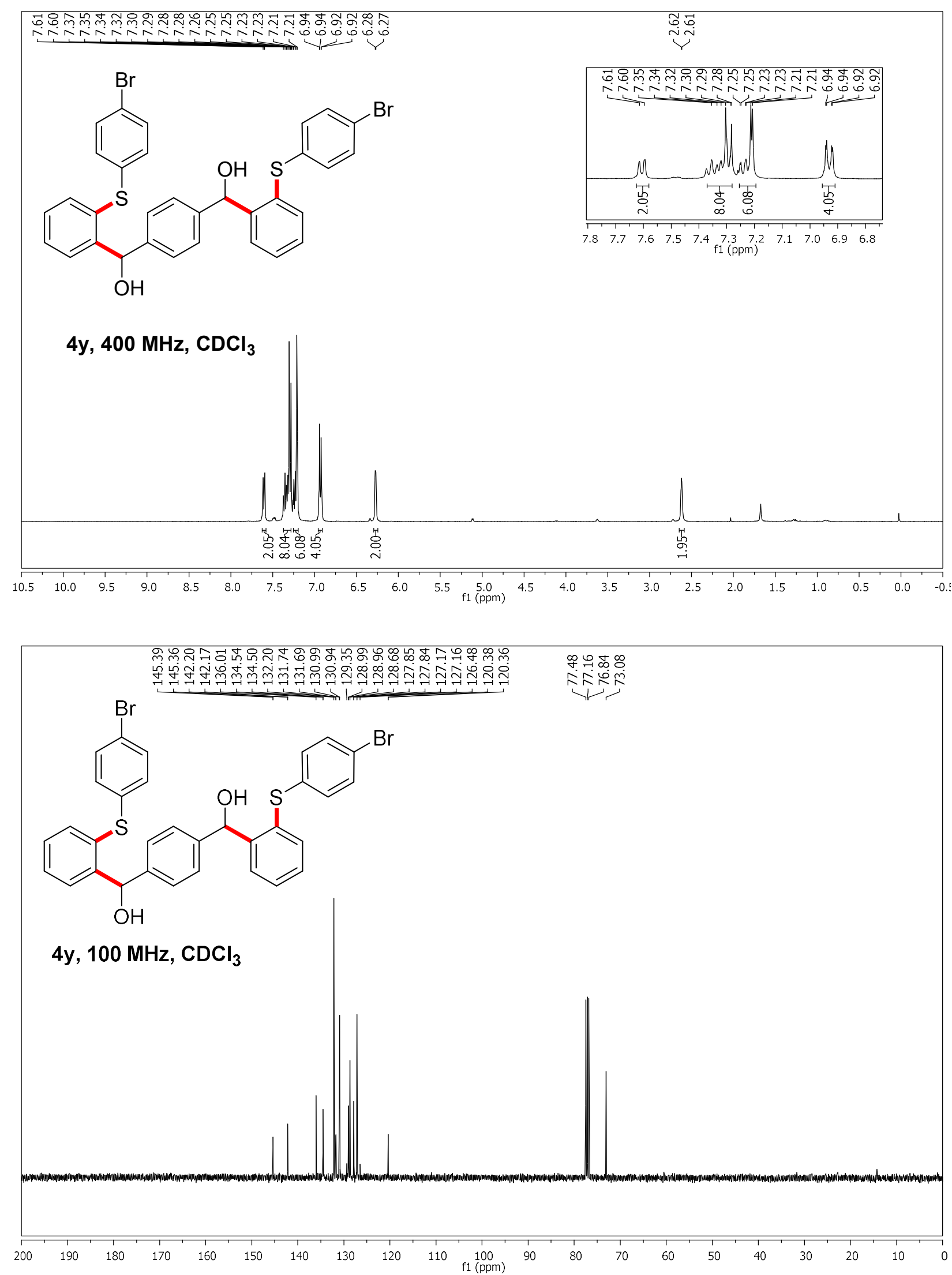
(4-Chlorophenyl)(2-(p-tolylthio)phenyl)methanol (4z)
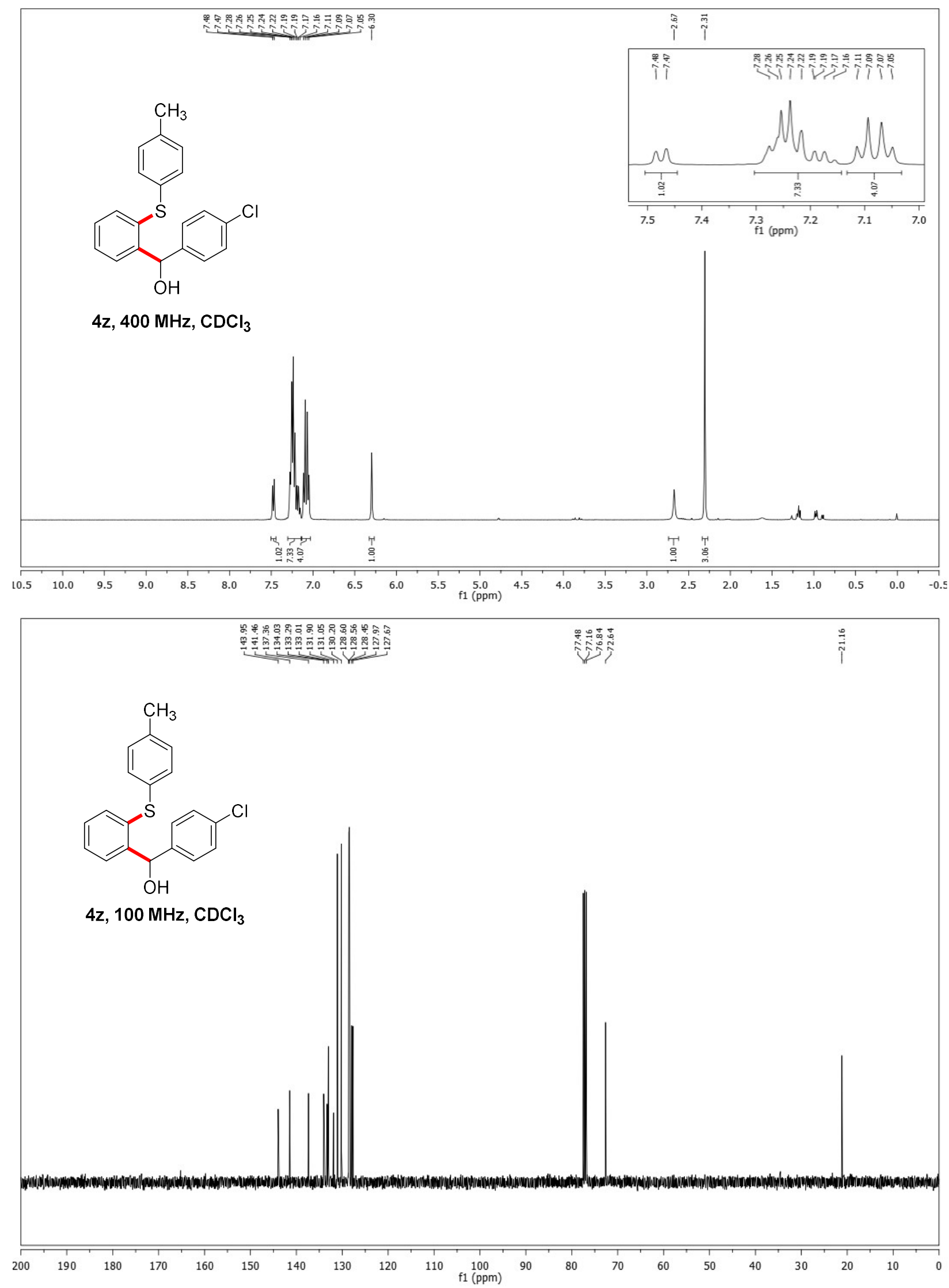
(4-Chlorophenyl)(2-(phenylthio)phenyl)methanol (4aa)
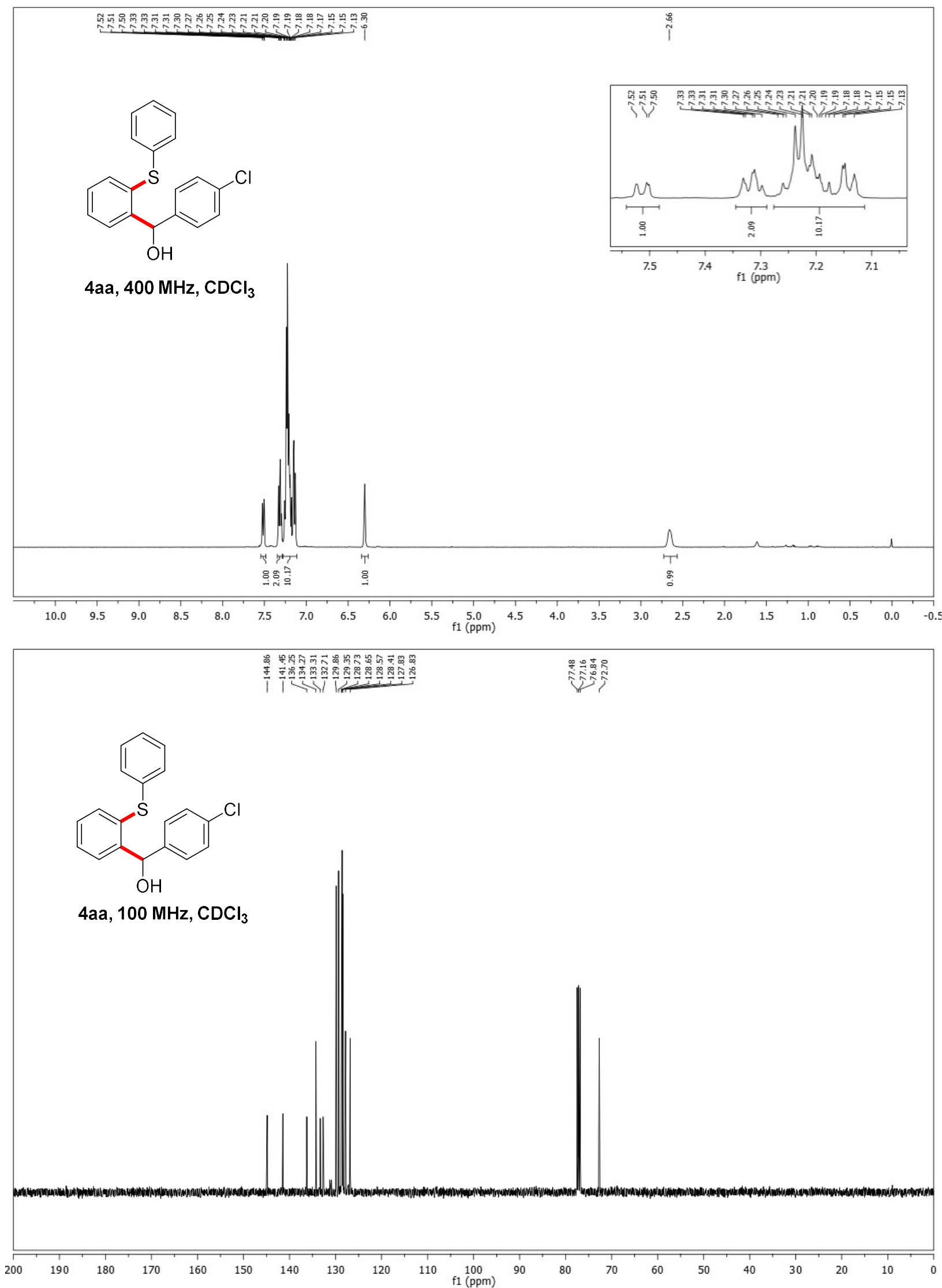
(4-Chlorophenyl)(2-((4-chlorophenyl)thio)phenyl)methanol (4ab)
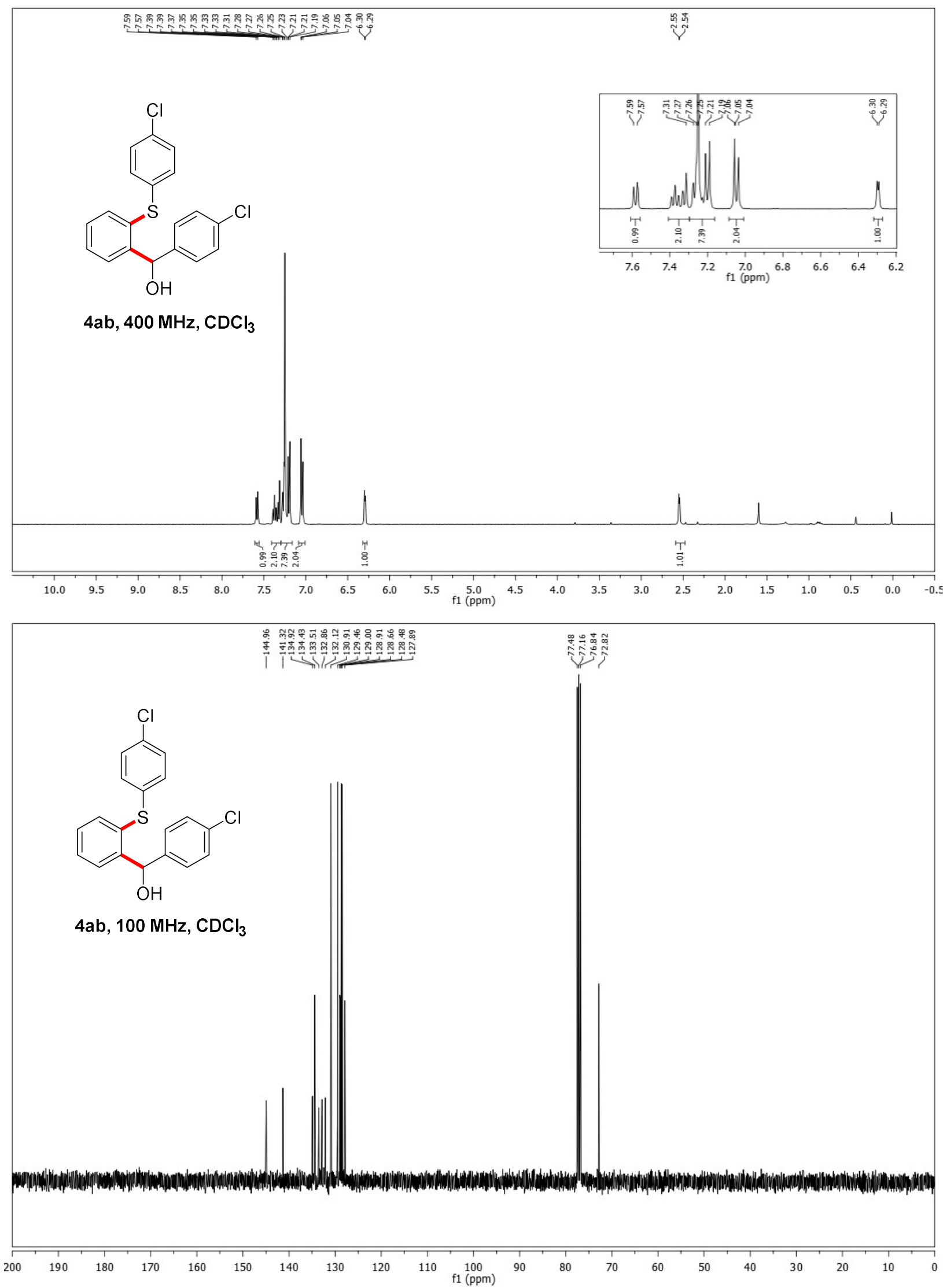
(4-Chlorophenyl)(2-((4-fluorophenyl)thio)phenyl)methanol (4ac)
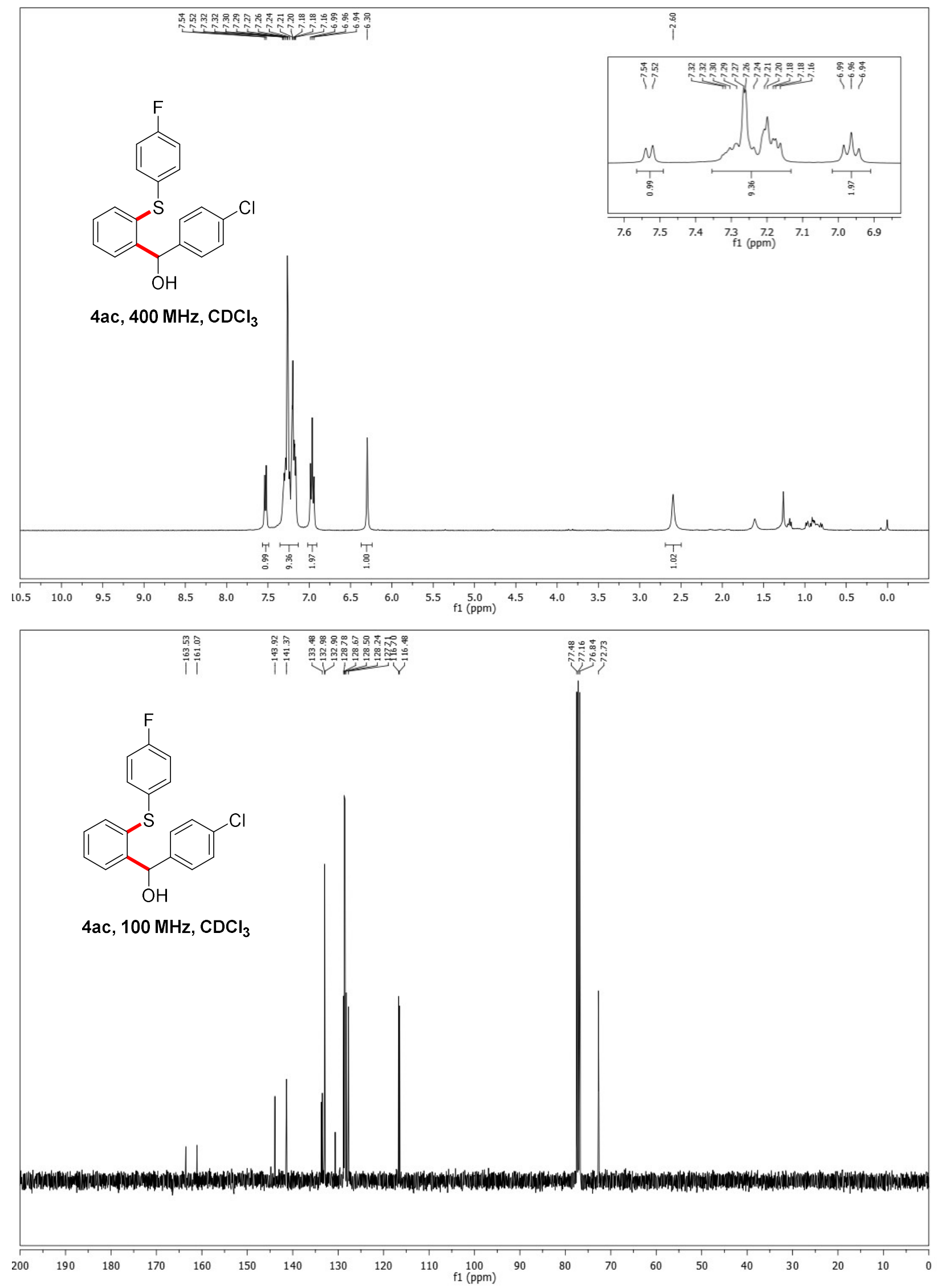
(4-Chlorophenyl)(2-((3-fluorophenyl)thio)phenyl)methanol (4ad)
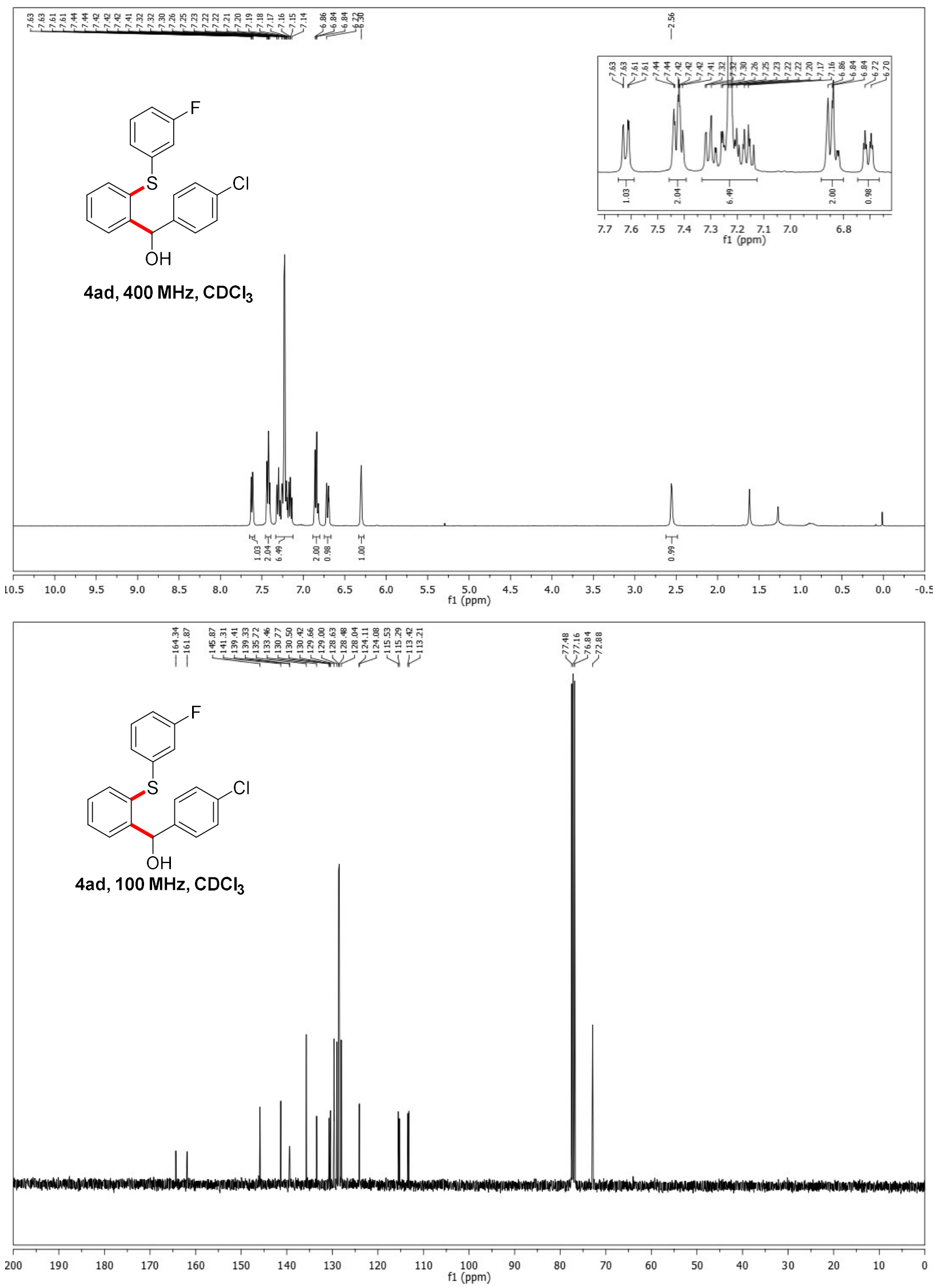
(2-((3-Bromophenyl)thio)phenyl)(4-chlorophenyl)methanol (4ae)
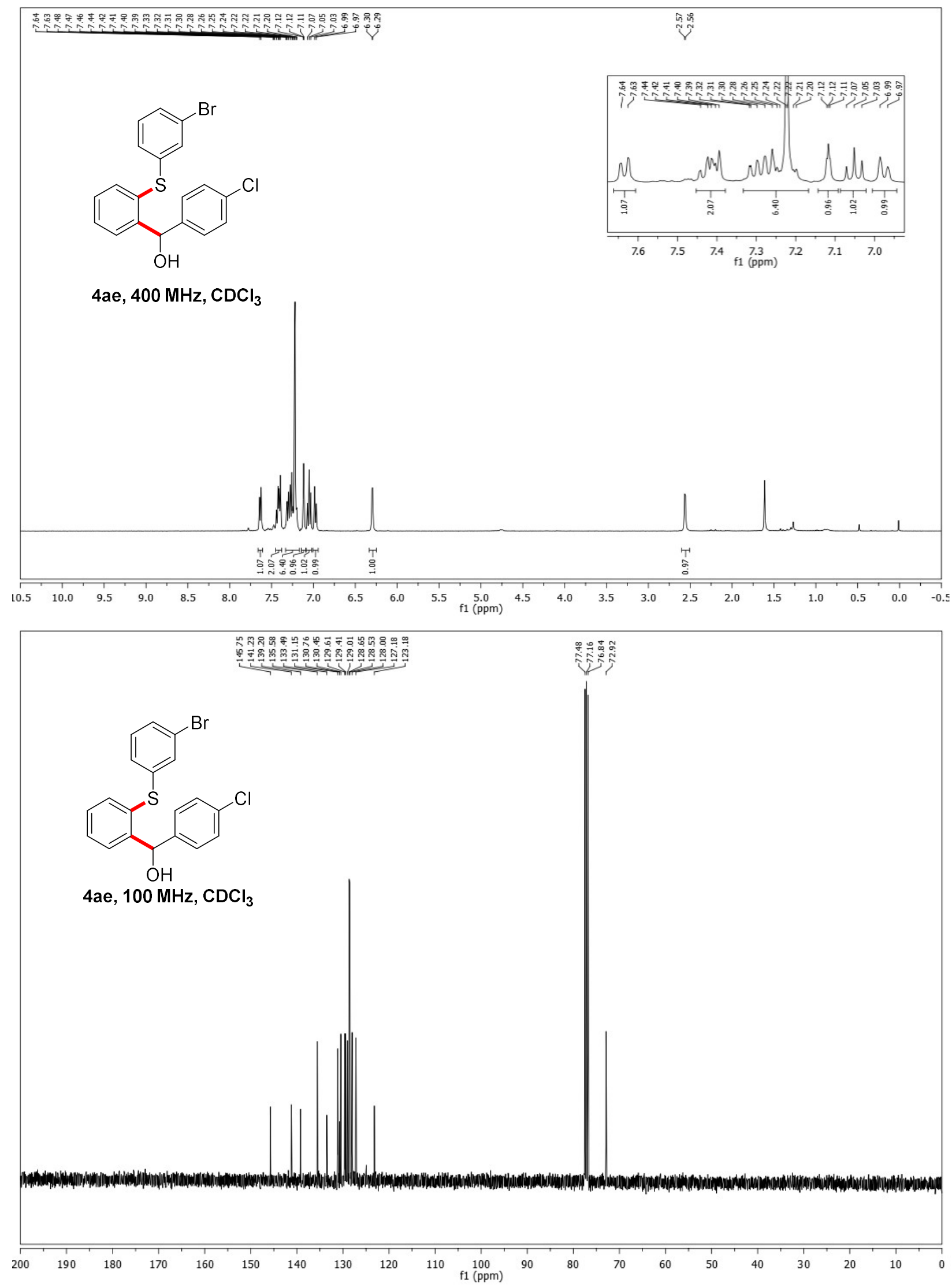
4-Chlorophenyl)(2-(o-tolylthio)phenyl)methanol (4af)
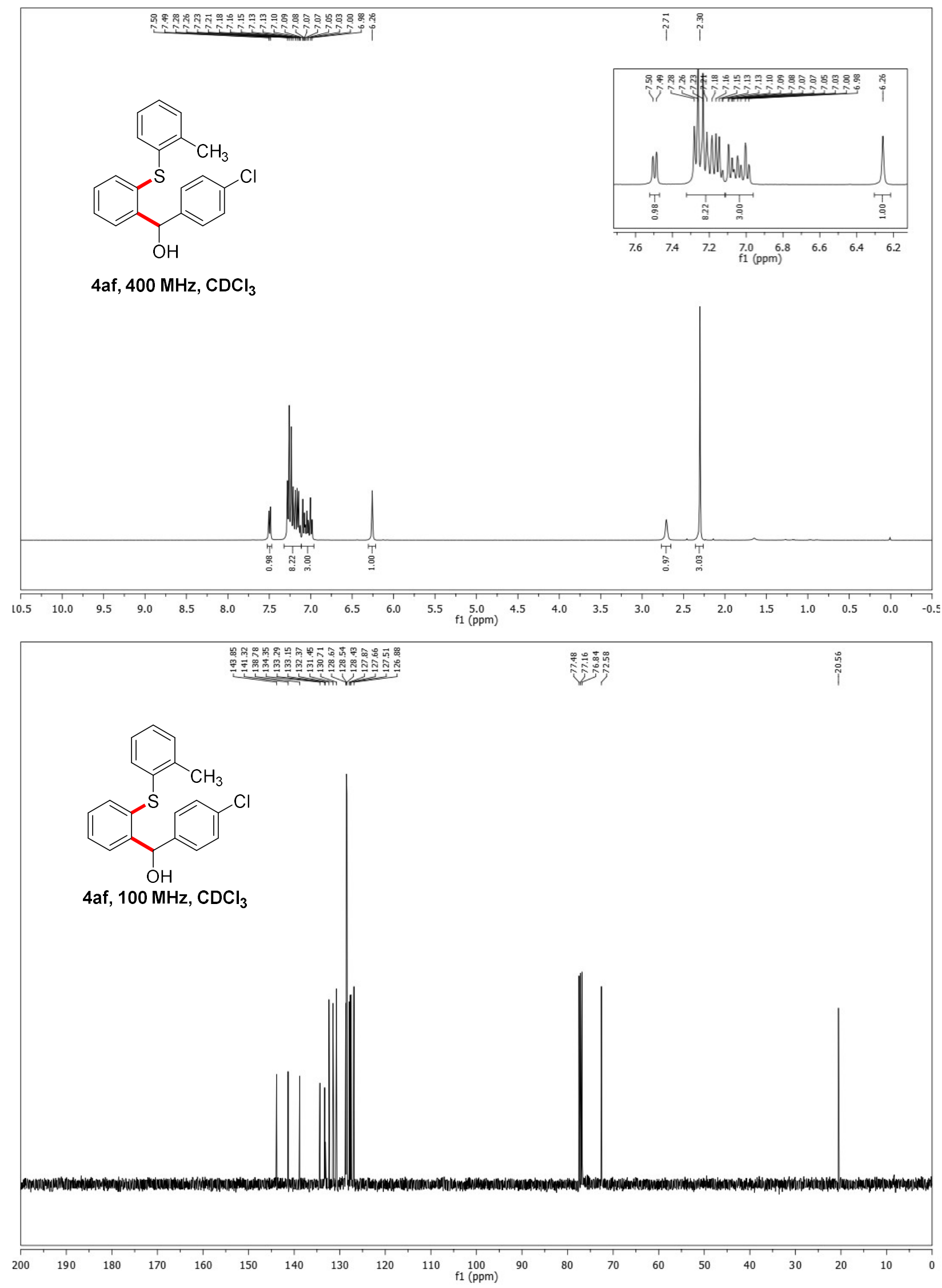
(4-Chlorophenyl)(2-((2-chlorophenyl)thio)phenyl)methanol (4ag)
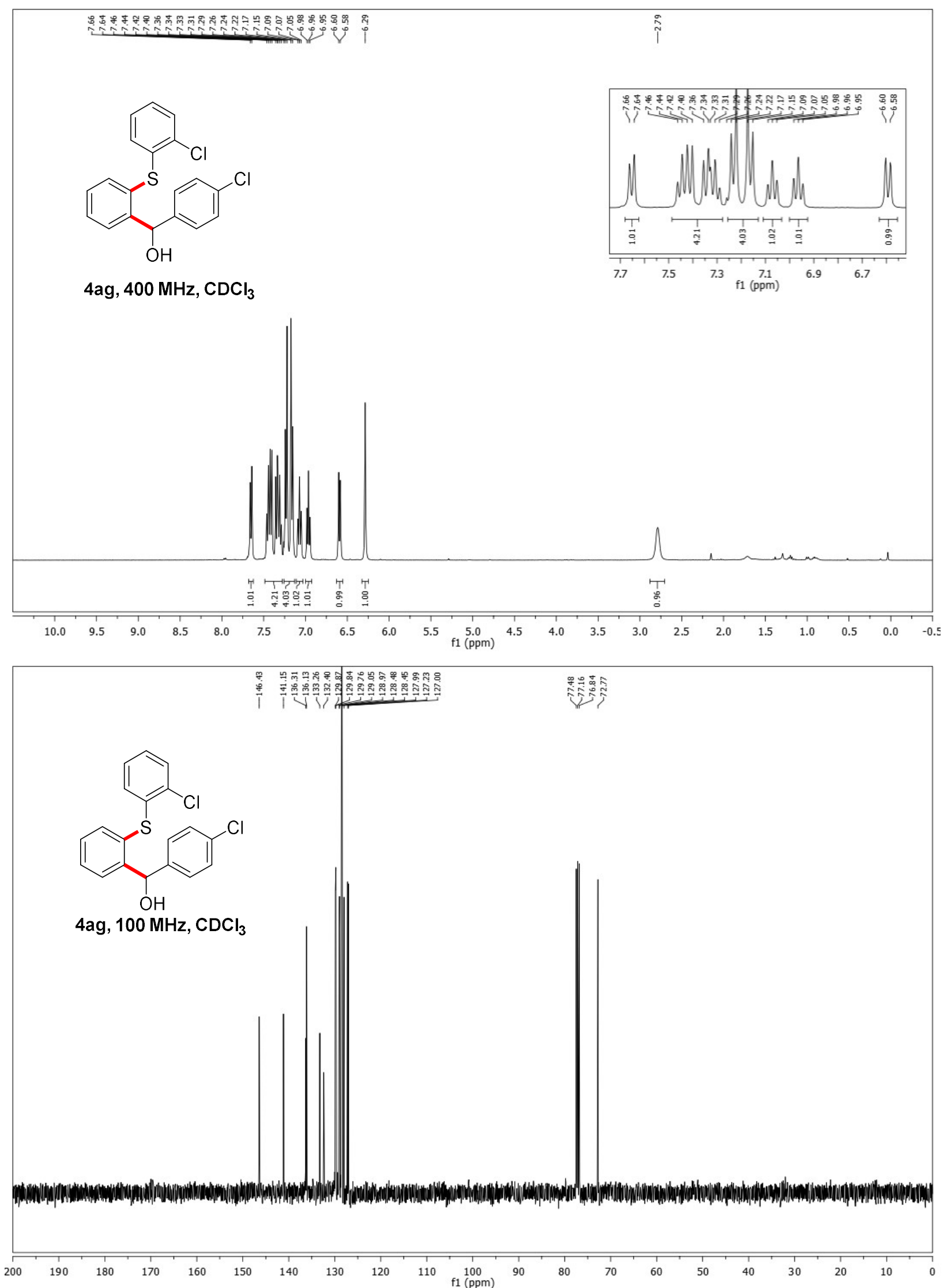
(4-Chlorophenyl)(2-((2-fluorophenyl)thio)phenyl)methanol (4ah)
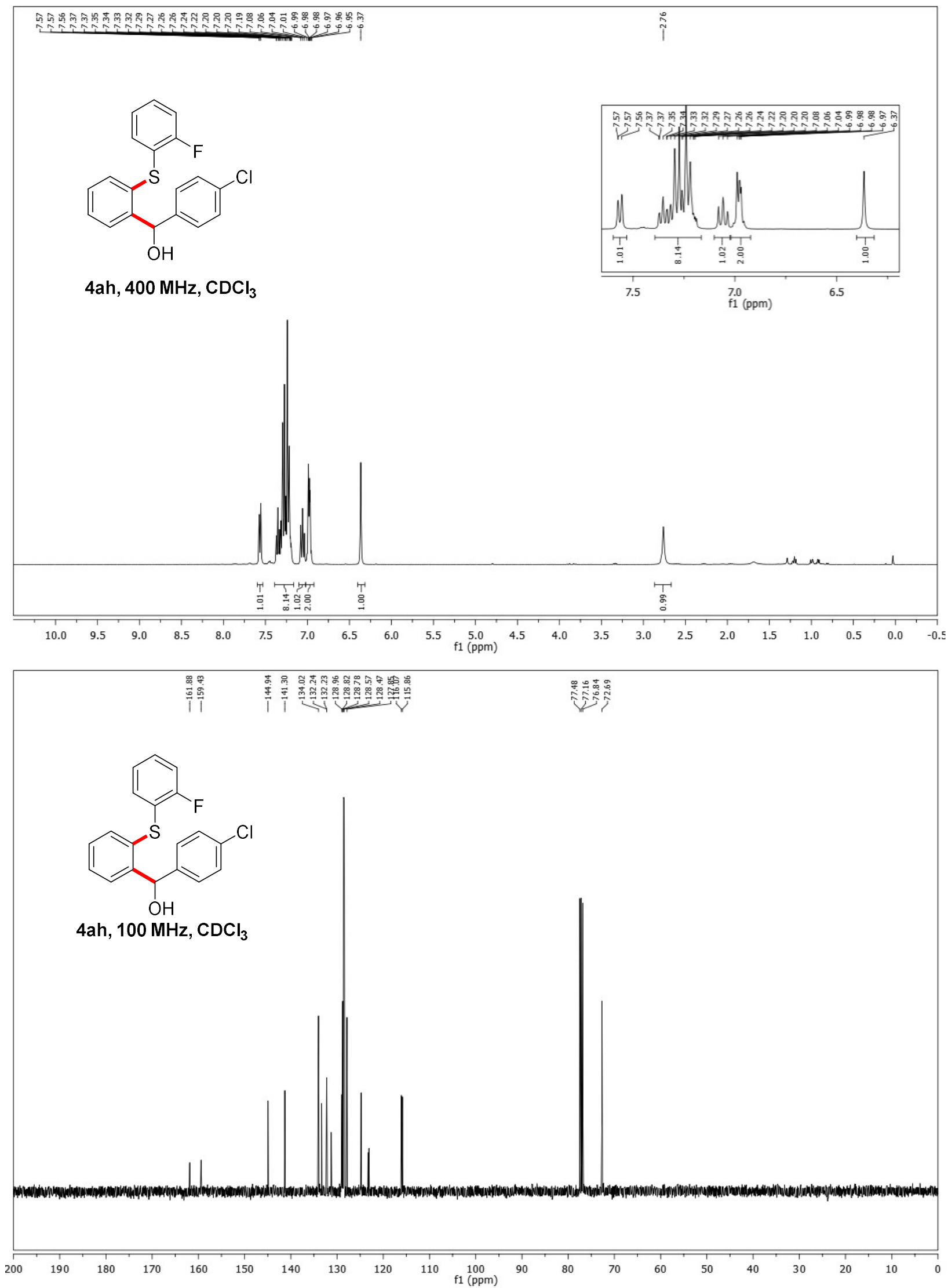
(4-Chlorophenyl)(2-(naphthalen-2-ylthio)phenyl)methanol (4ai)
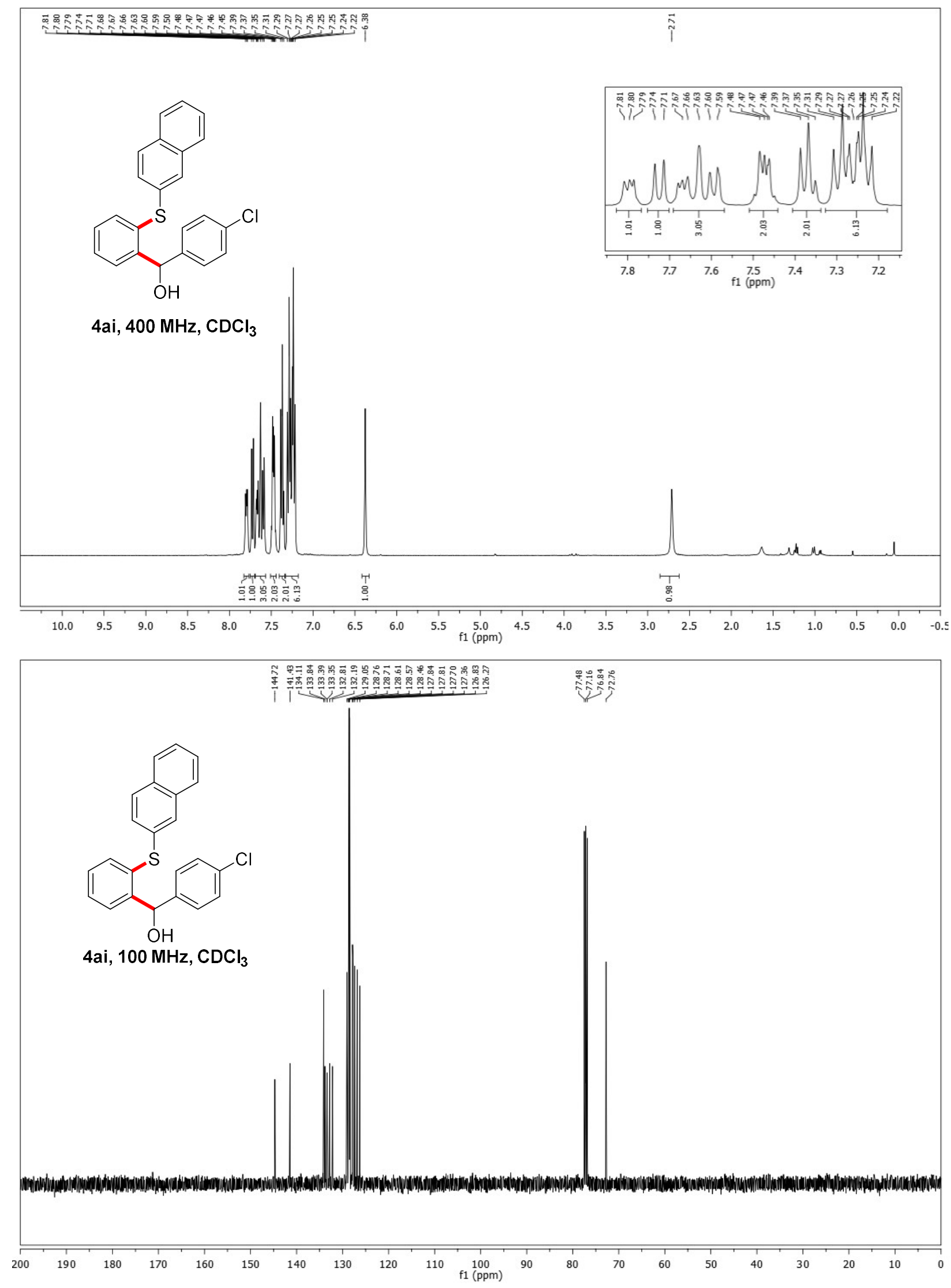
(2-((4-Bromophenyl)thio)-4,5-dimethylphenyl)(4-chlorophenyl)methanol (4aj)
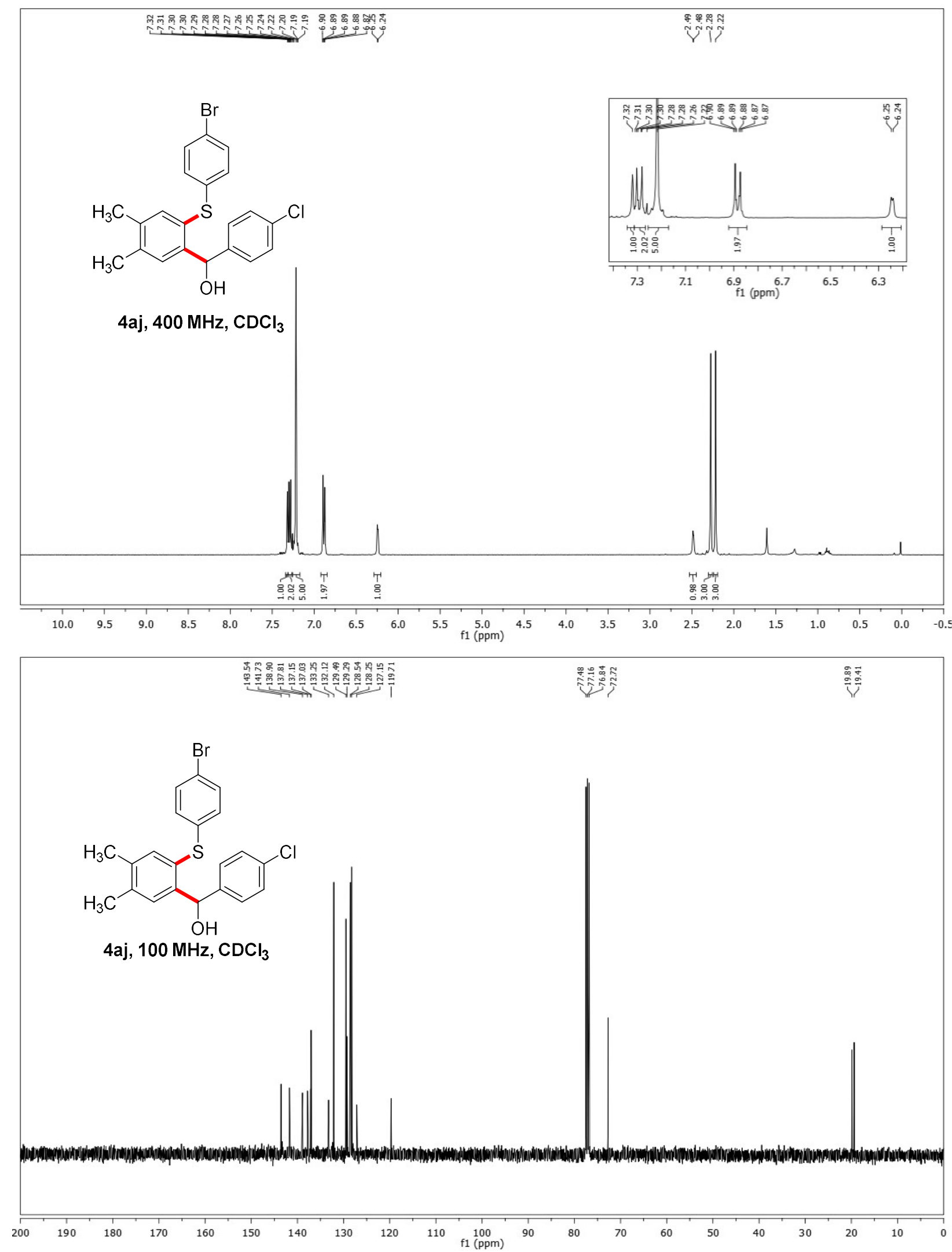
(6-((4-Bromophenyl)thio)benzo[d][1,3]dioxol-5-yl)(4-chlorophenyl)methanol (4ak)
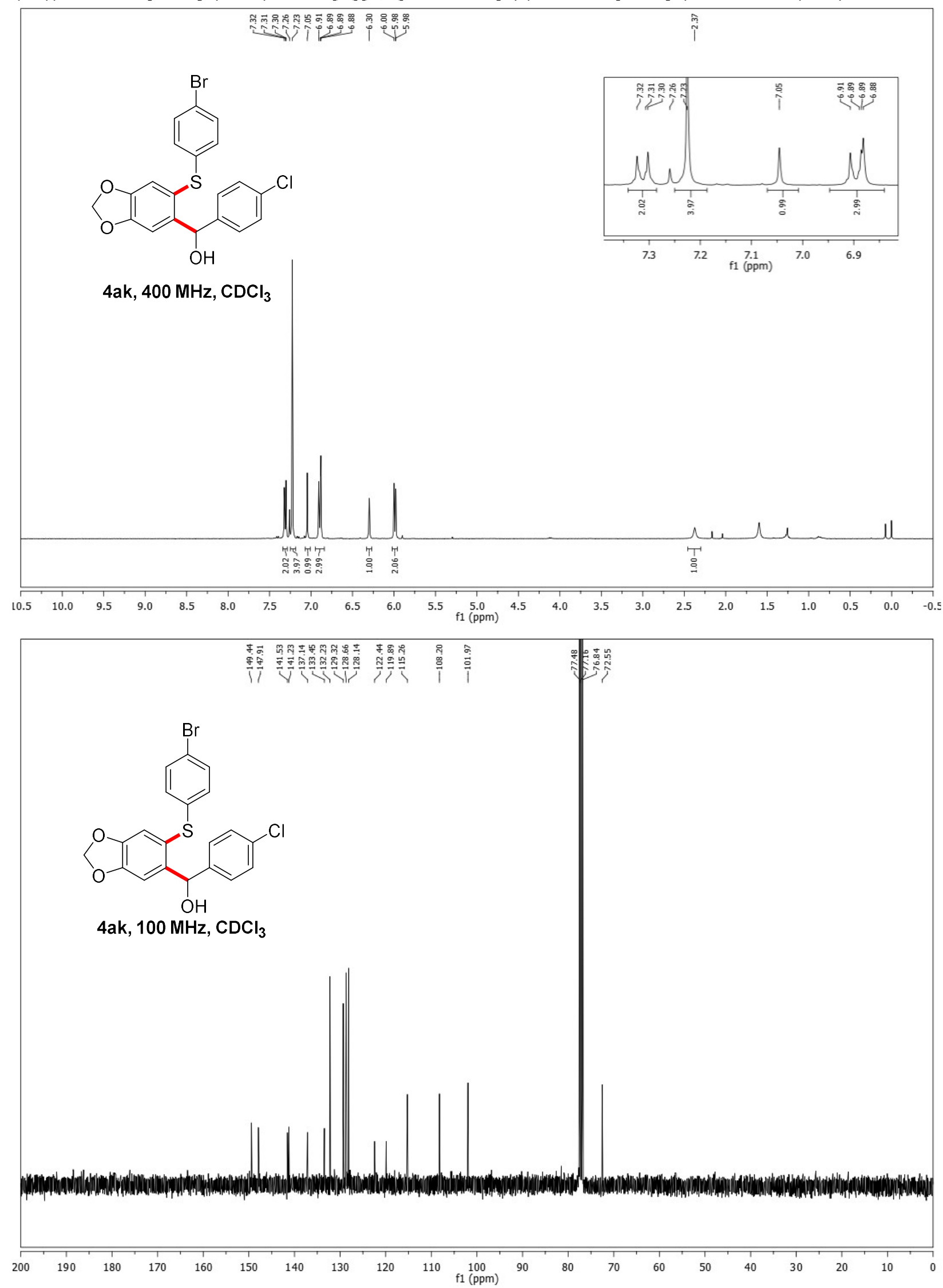
(6-((4-Bromophenyl)thio)-2,3-dihydro-1H-inden-5-yl)(4-chlorophenyl)methanol (4al)
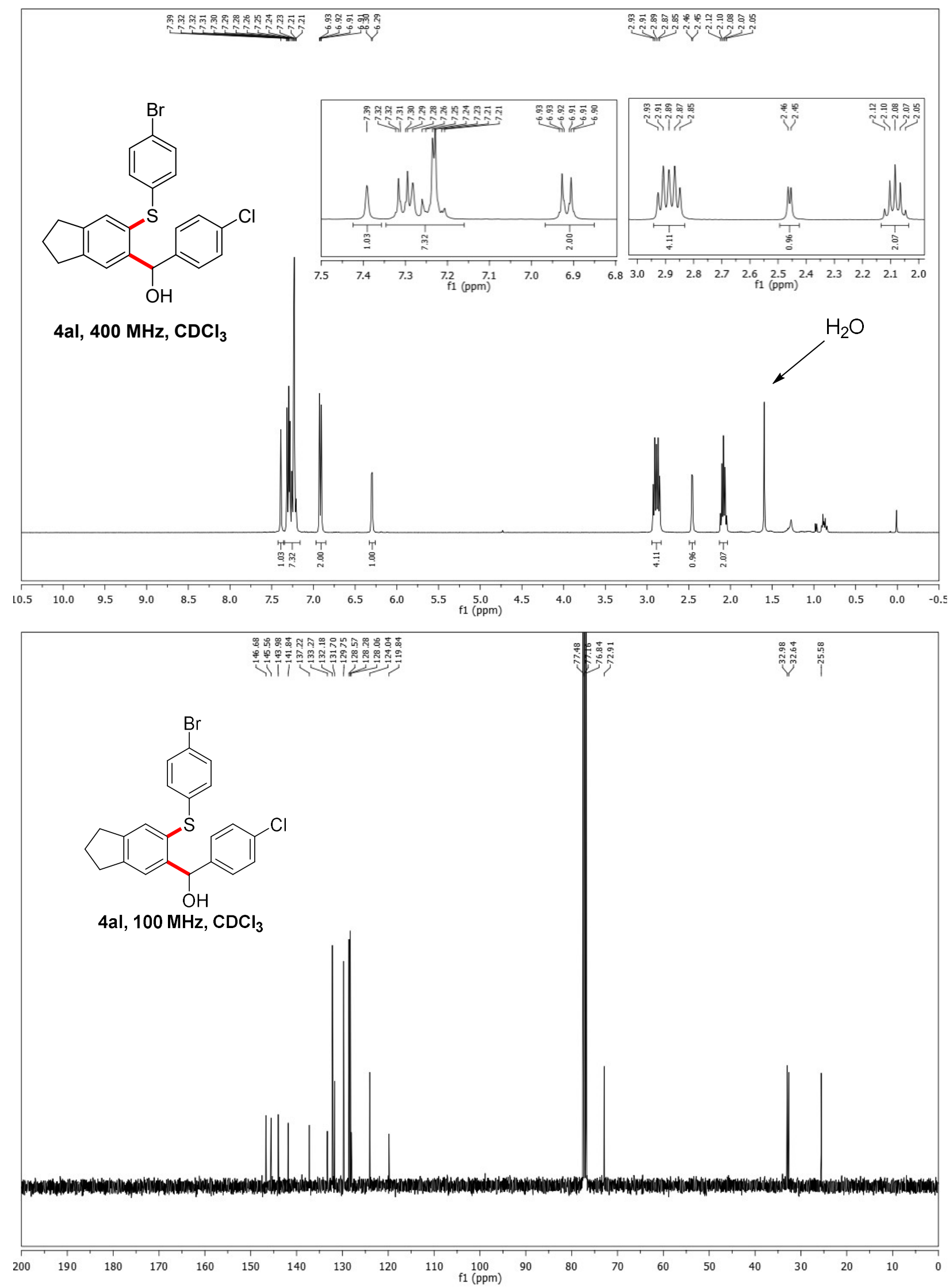
(2-((4-Bromophenyl)thio)-4,5-difluorophenyl)(4-chlorophenyl)methanol (4am)
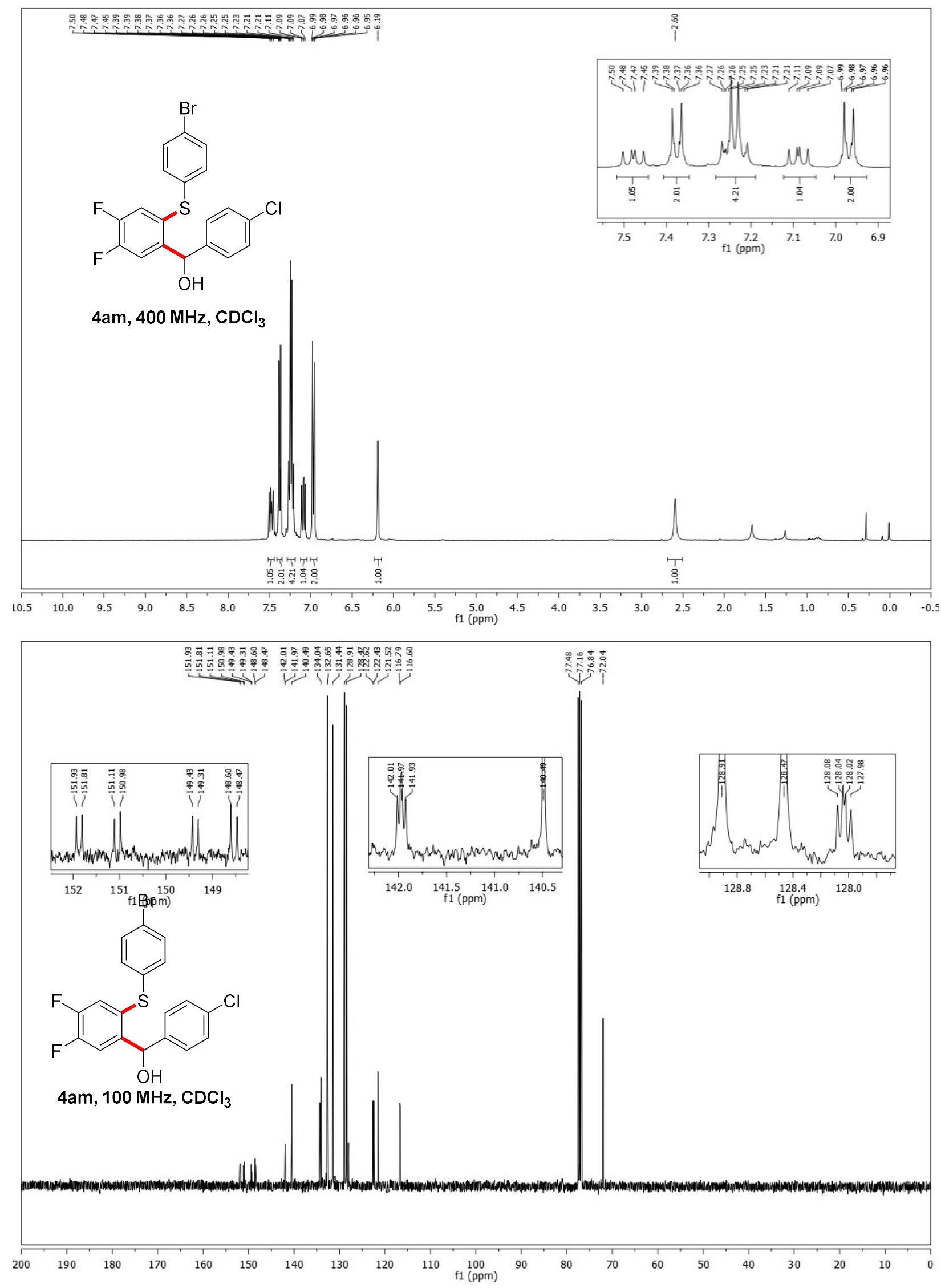
(2-((4-Bromophenyl)thio)-3,6-dimethylphenyl)(4-chlorophenyl)methanol (4an)
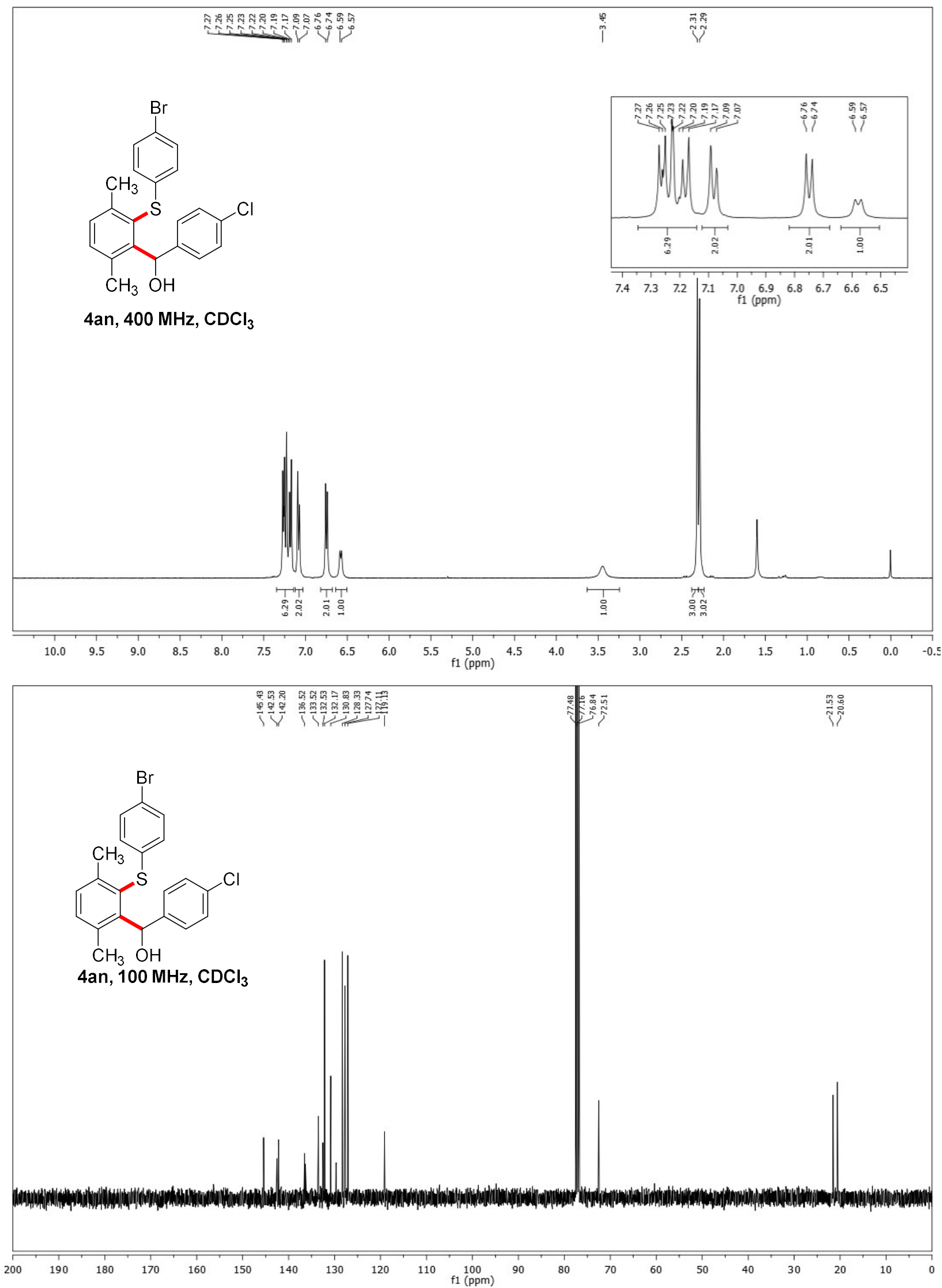
(3-((4-Bromophenyl)thio)naphthalen-2-yl)(4-chlorophenyl)methanol (4ao)
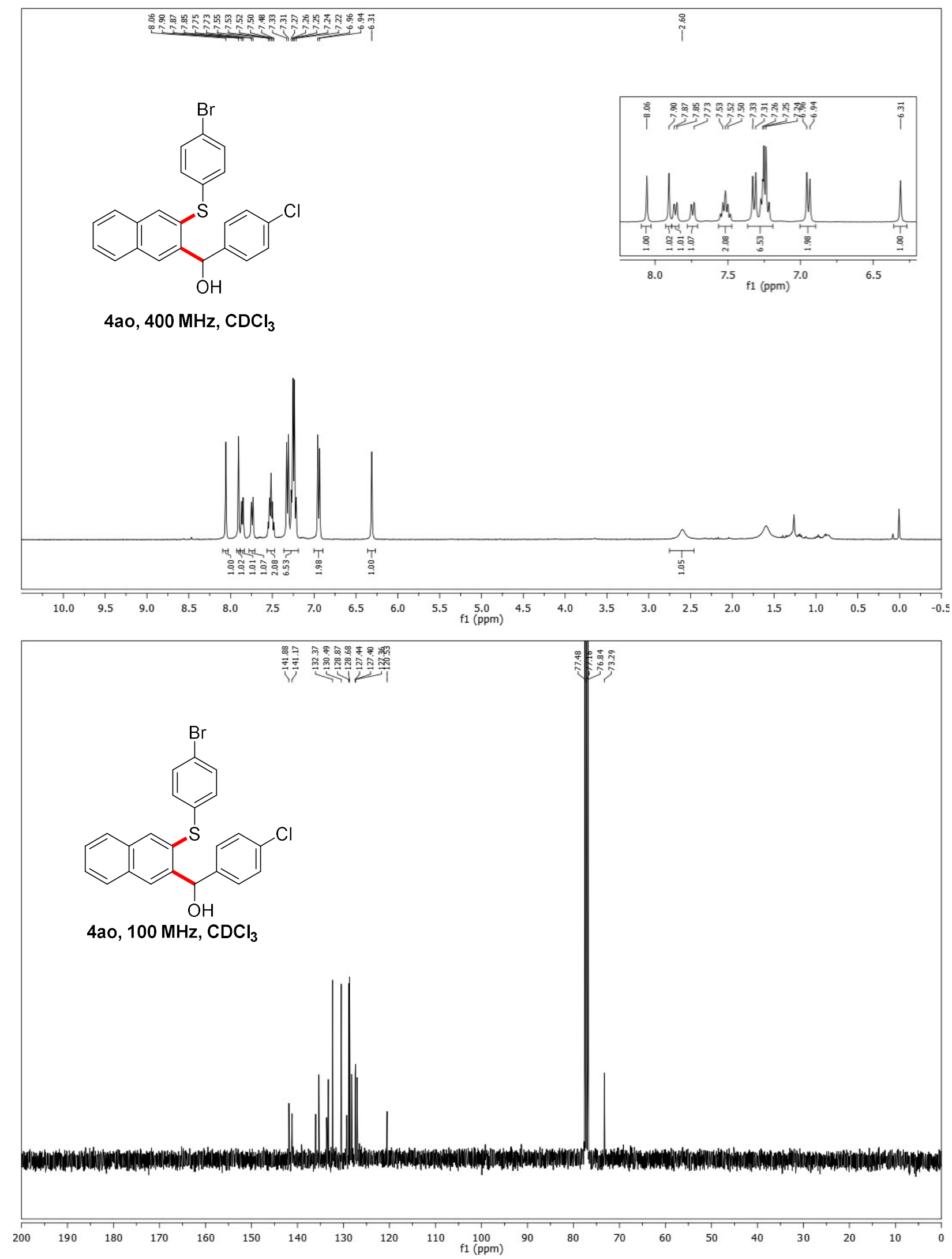
(1-((4-Bromophenyl)thio)naphthalen-2-yl)(4-chlorophenyl)methanol (4ap)
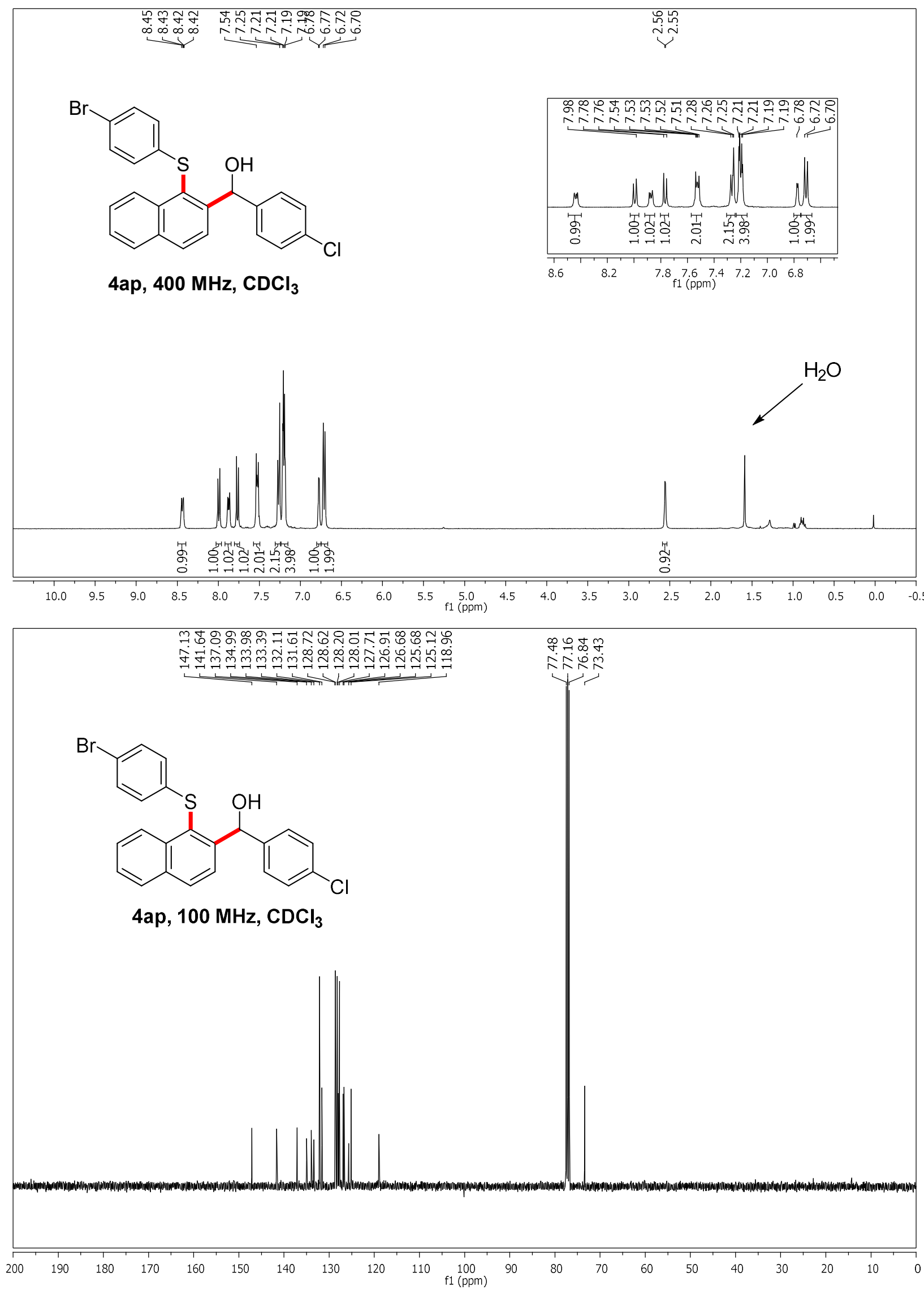

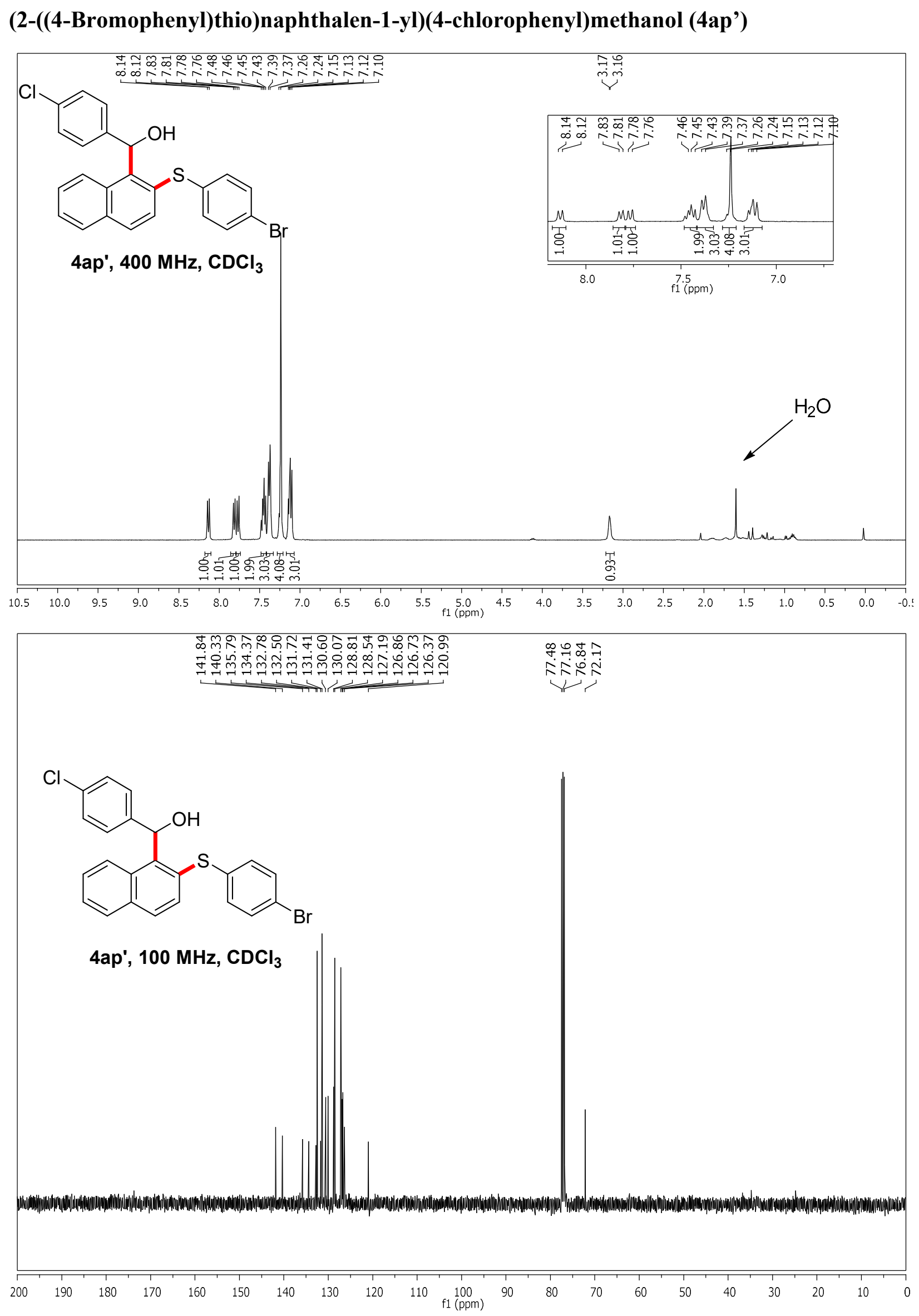

S79 
(2-((4-Bromophenyl)thio)-4-methylphenyl)(4-chlorophenyl)methanol (4aq) and

( 2-((4-Bromophenyl)thio)-5-methylphenyl)(4-chlorophenyl)methanol (4aq')
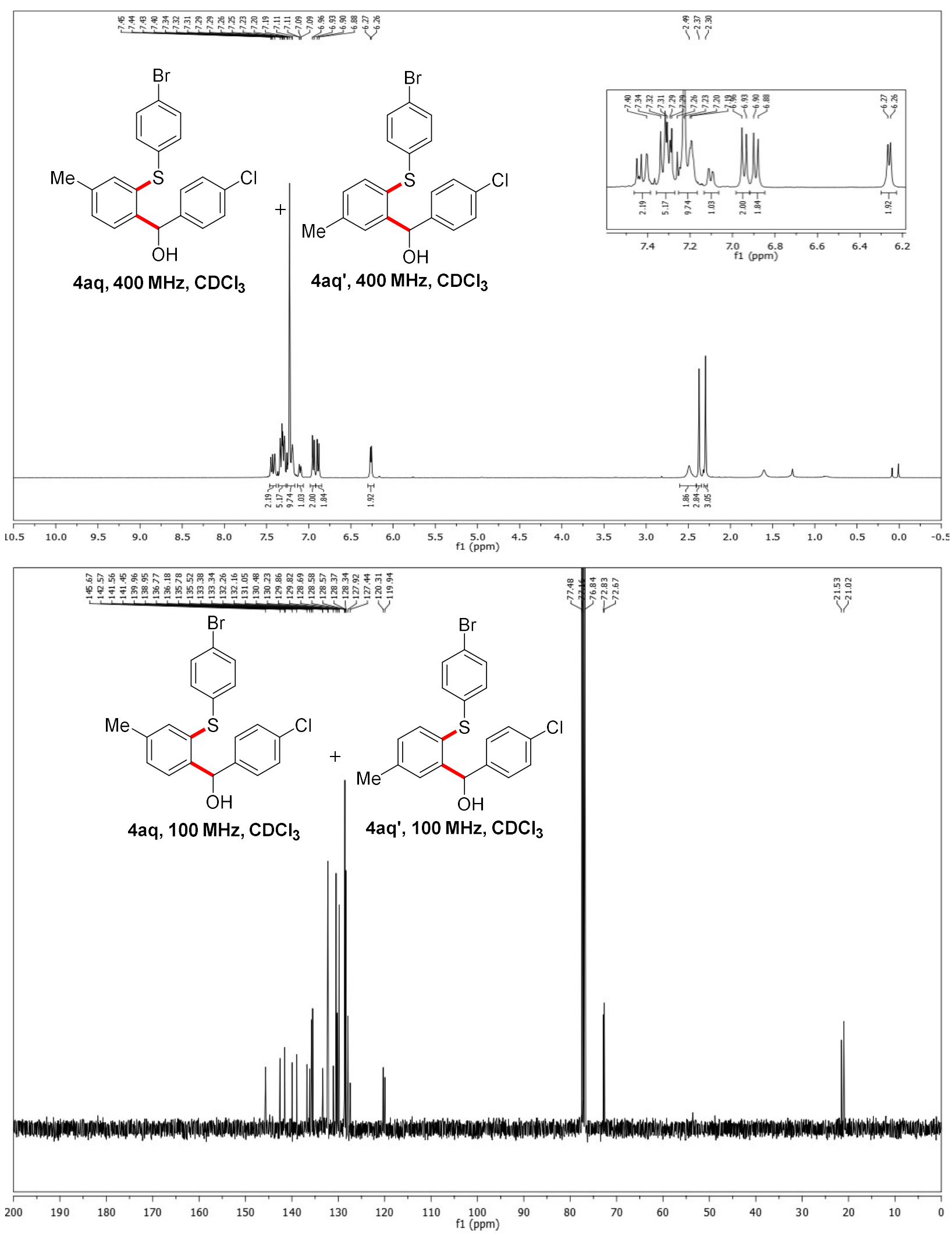
(2-((4-Bromophenyl)thio)-4-fluorophenyl)(4-chlorophenyl)methanol (4ar) and (2-((4-Bromophenyl)thio)-5-fluorophenyl)(4-chlorophenyl)methanol (4ar')
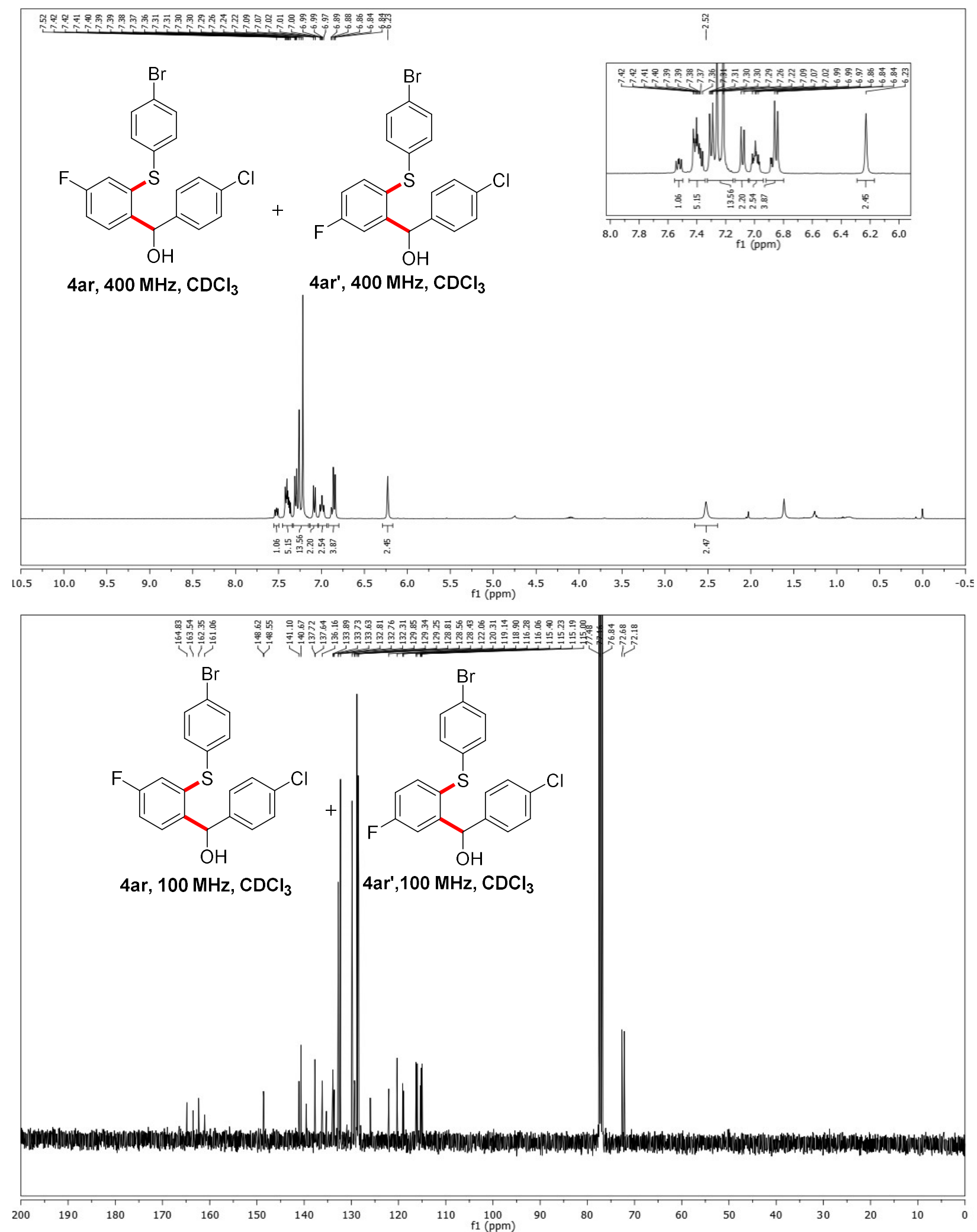
Ethyl 2-(2-((4-bromophenyl)thio)phenyl)-2-hydroxy-2-phenylacetate (6a)
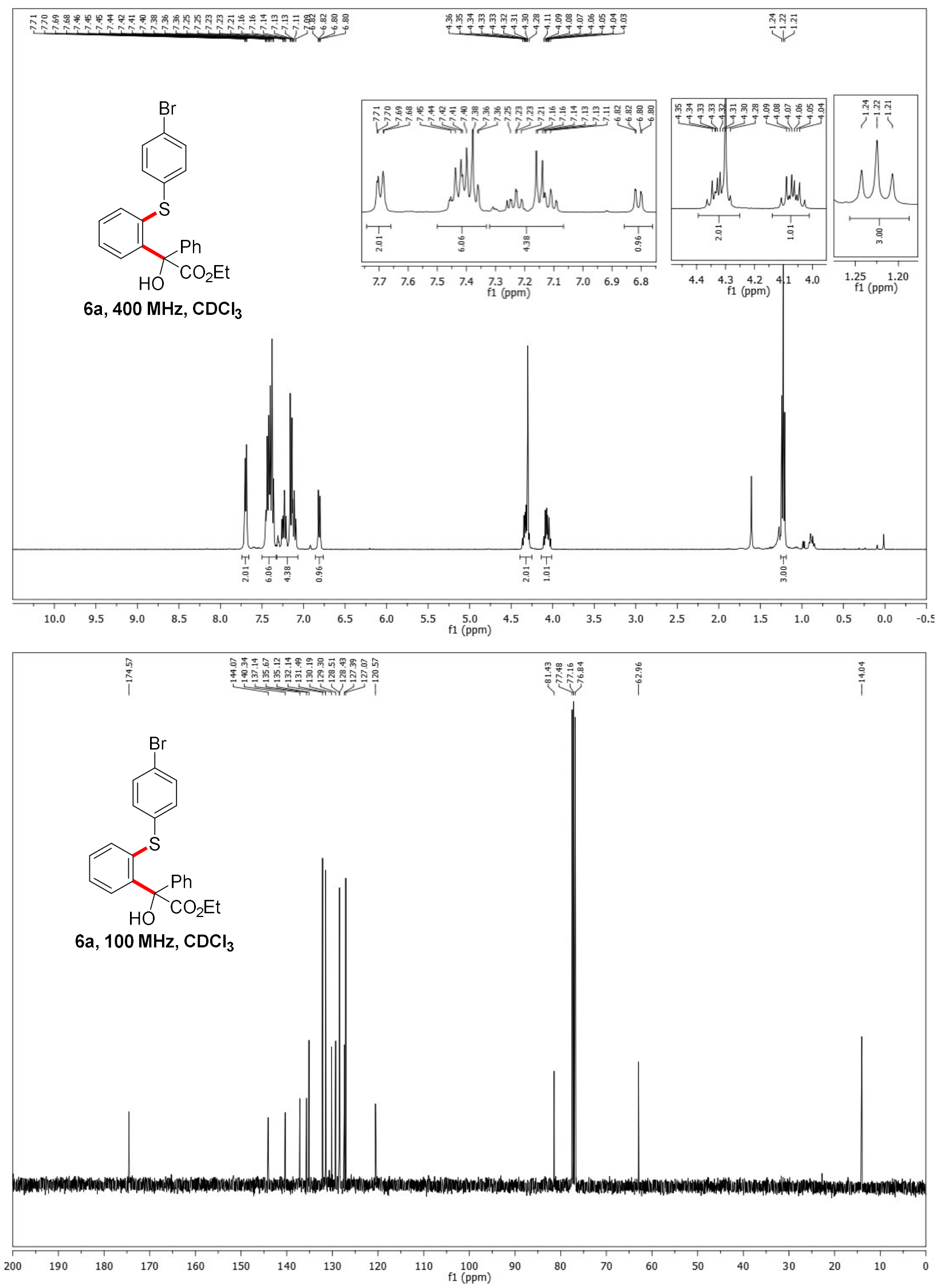
1-(2-((4-Bromophenyl)thio)phenyl)-2,2,2-trifluoro-1-phenylethan-1-ol (8a)
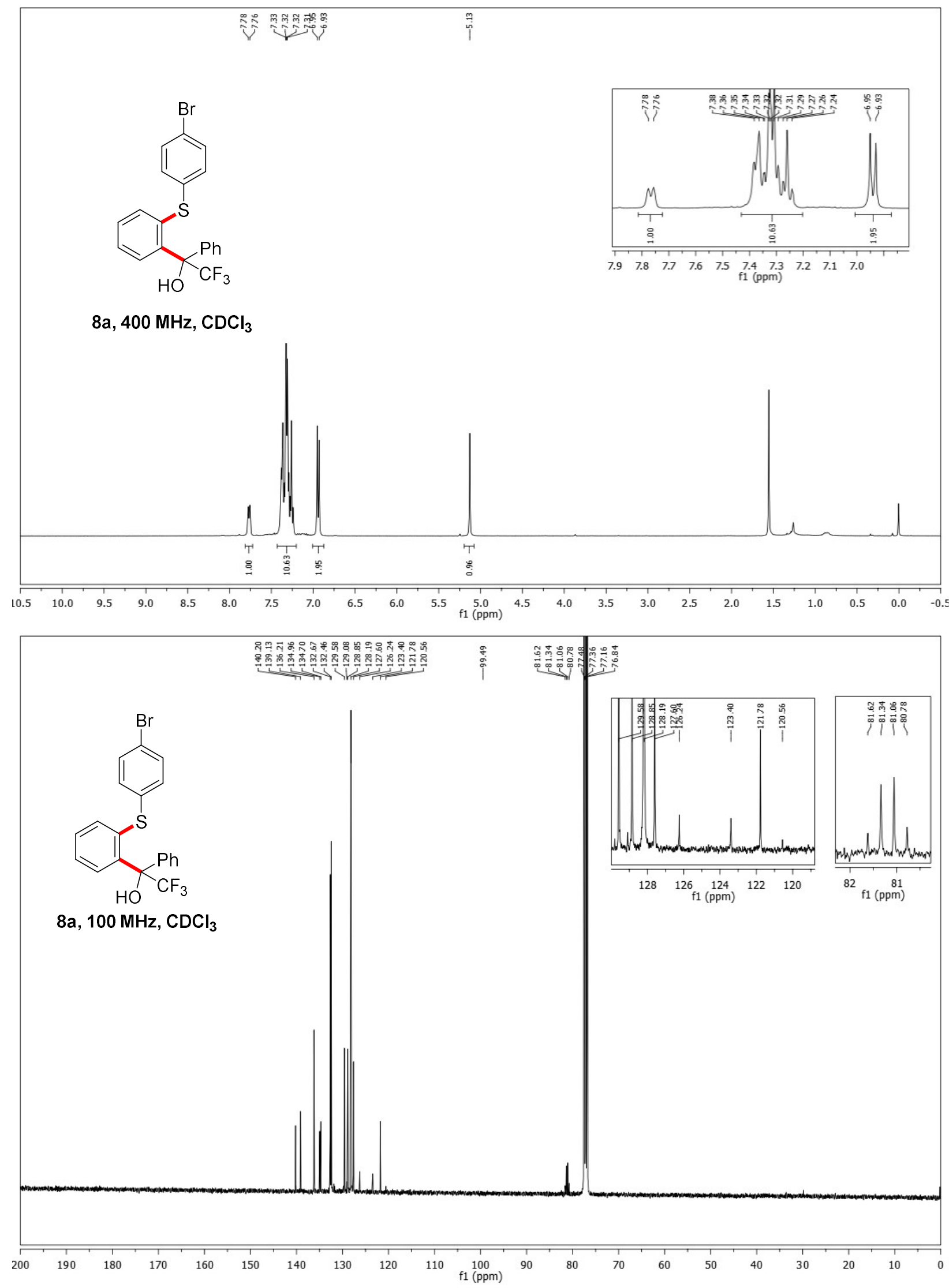
(2-((4-Bromophenyl)thio)phenyl)(4-chlorophenyl)methanol (10a)
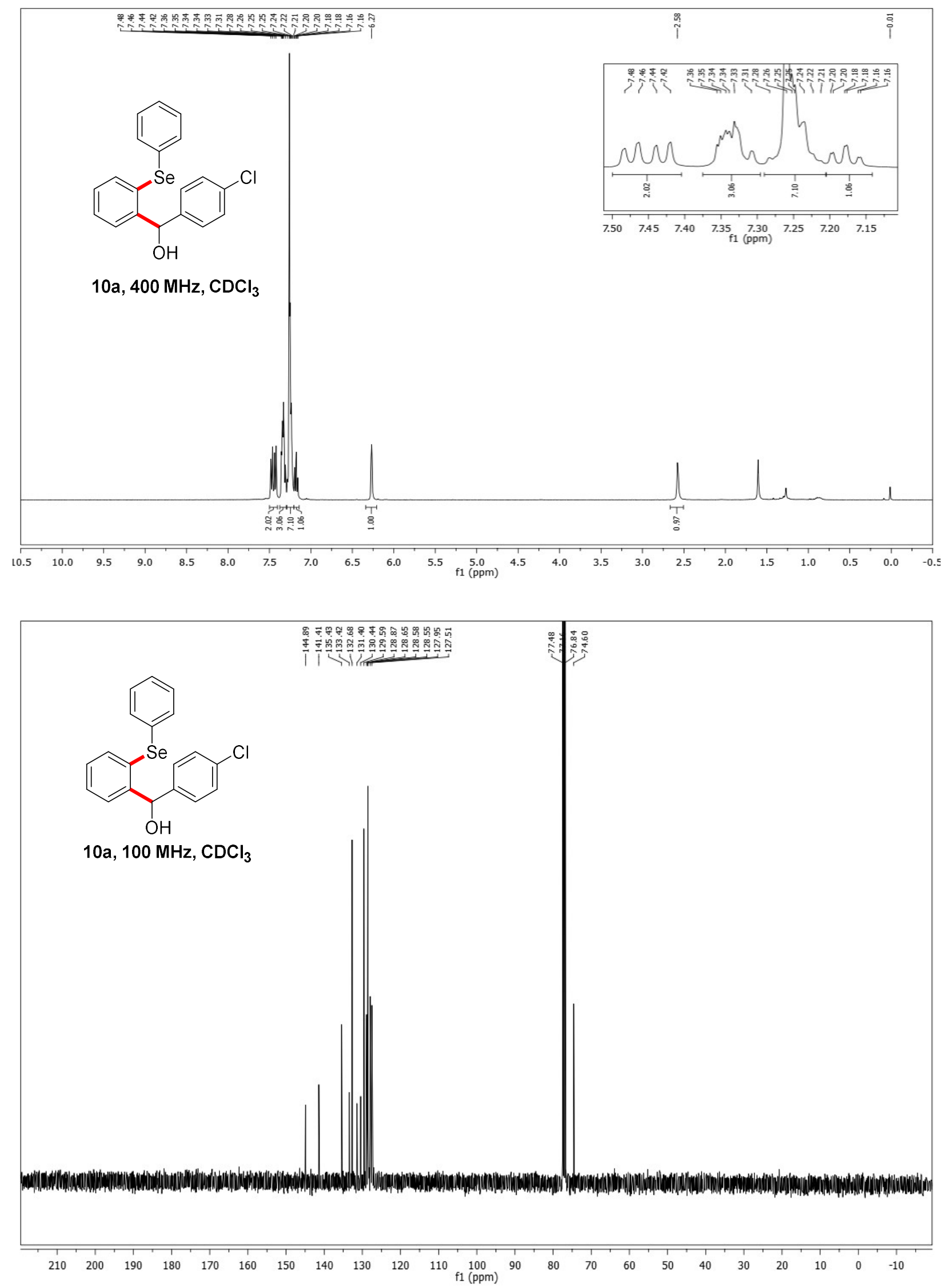
2-Bromo-9-(4-chlorophenyl)-9H-thioxanthene (11a)
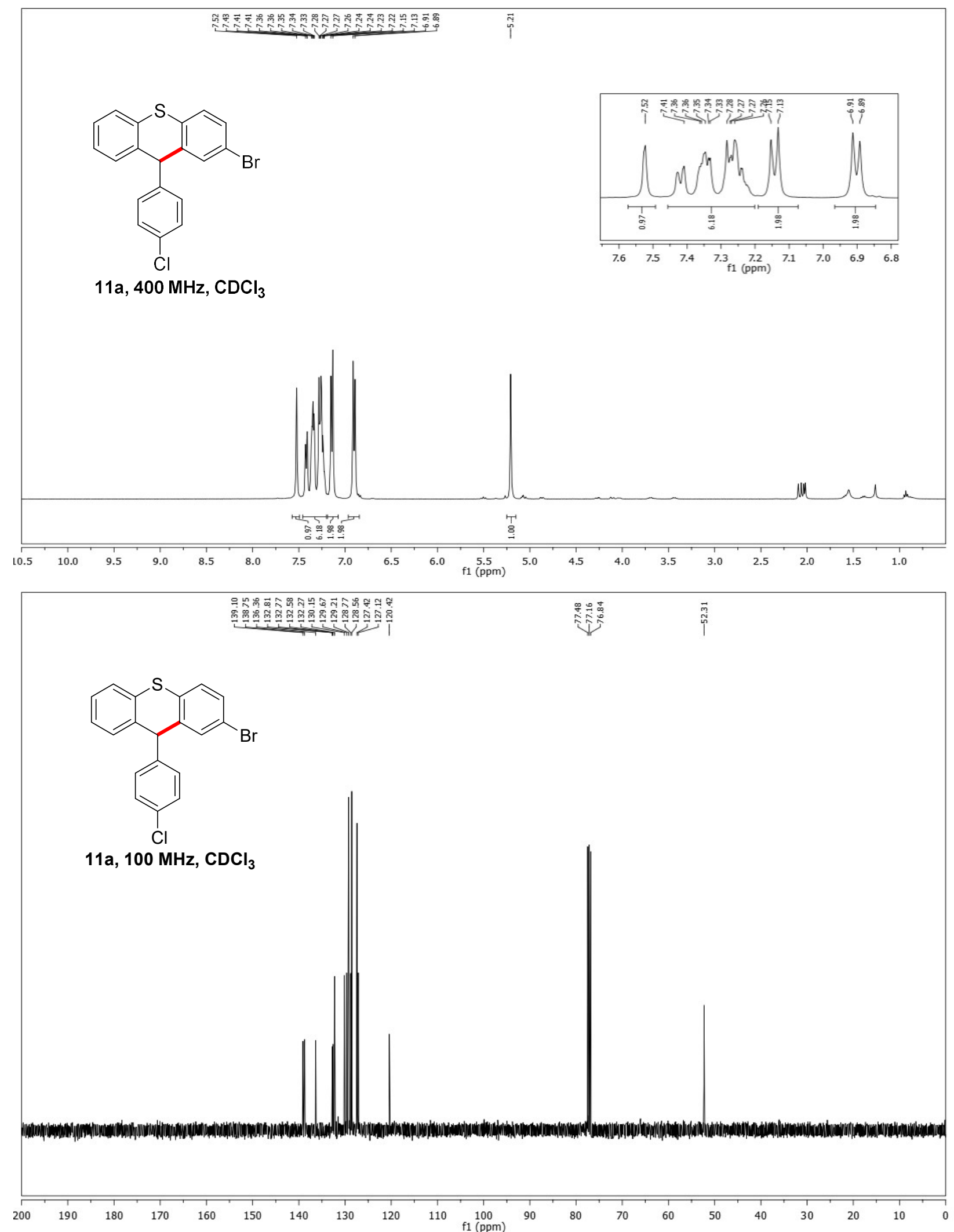